\title{
Search for microscopic black holes in a like-sign dimuon final state using large track multiplicity with the ATLAS detector
}

\author{
G. Aad et al.* \\ (ATLAS Collaboration)
}

(Received 19 August 2013; published 1 October 2013)

\begin{abstract}
A search is presented for microscopic black holes in a like-sign dimuon final state in proton-proton collisions at $\sqrt{s}=8 \mathrm{TeV}$. The data were collected with the ATLAS detector at the Large Hadron Collider in 2012 and correspond to an integrated luminosity of $20.3 \mathrm{fb}^{-1}$. Using a high track multiplicity requirement, $0.6 \pm 0.2$ background events from Standard Model processes are predicted and none observed. This result is interpreted in the context of low-scale gravity models and 95\% C.L. lower limits on microscopic black hole masses are set for different model assumptions.
\end{abstract}

DOI: 10.1103/PhysRevD.88.072001

\section{INTRODUCTION}

The hierarchy problem, in which the Planck scale $\left(M_{\mathrm{Pl}} \approx 10^{19} \mathrm{GeV}\right)$ is much higher than the electroweak scale $(\approx 100 \mathrm{GeV})$, provides a strong motivation to search for new phenomena not described by the Standard Model of particle physics. A theory introducing extra dimensions is one possible solution. In some models of extra dimensions, the gravitational field propagates into $n+4$ dimensions, where $n$ is the number of extra dimensions beyond the four space-time dimensions. One of the models of extra dimensions is the model proposed by Arkani-Hamed, Dimopoulos, and Dvali (ADD) [1-3], in which the gravitational field propagates into large, flat, extra dimensions while the Standard Model particles are localized in four space-time dimensions. Since the gravitational field propagates into the extra dimensions, it is measured at a reduced strength in the four space-time dimensions. Thus, the fundamental Planck scale in $D=4+n$ dimensions, $M_{\mathrm{D}}$, could be comparable with the electroweak scale.

If extra dimensions exist and $M_{\mathrm{D}}$ is of the order of $1 \mathrm{TeV}$, microscopic black holes with $\mathrm{TeV}$-scale mass could exist and be produced at the Large Hadron Collider (LHC) [4-8]. These black holes are produced when the impact parameter of the two colliding protons is smaller than the higherdimensional event horizon of a black hole with mass equal to the invariant mass of the colliding proton system.

The black hole production has a continuous mass distribution ranging from $M_{\mathrm{D}}$ to the proton-proton center-ofmass energy. The black holes evaporate by emitting Hawking radiation [9], which determines the energy and multiplicity of the emitted particles. The relative multiplicities of different particle types are determined by the

*Full author list given at the end of the article.

Published by the American Physical Society under the terms of the Creative Commons Attribution 3.0 License. Further distribution of this work must maintain attribution to the author(s) and the published article's title, journal citation, and DOI.
PACS numbers: $13.85 . \mathrm{Rm}, 04.50 .-\mathrm{h}, 04.50 . \mathrm{Gh}, 11.10 . \mathrm{Kk}$

number of degrees of freedom of each particle type and the decay modes of the emitted unstable particles. Black hole events are thus expected to have a high multiplicity of high-momentum particles.

This paper describes a search for black holes in a likesign dimuon final state. This final state can arise from muons directly produced by the black hole, or from the decay of Standard Model particles produced by the black hole. The final state is expected to have low Standard Model backgrounds while retaining a high signal acceptance. Since the microscopic black holes can decay to a large number of particles with high transverse momentum $\left(p_{\mathrm{T}}\right)$, the total track multiplicity of the event is exploited to distinguish signal events from backgrounds. The final result is obtained from the event yield in a signal region defined by high track multiplicity.

The following assumptions and conventions apply in this analysis. The classical approximations used for black hole production and the semiclassical approximations for the decay are predicted to be valid only for energies and black hole masses well above $M_{\mathrm{D}}$. In order to reduce the importance of the kinematic region where the incoming quark or gluon energy is low, a conservative assumption is made and a lower threshold $\left(M_{\mathrm{TH}}\right)$ is applied to the black hole mass, $M_{\mathrm{TH}}>M_{\mathrm{D}}+0.5 \mathrm{TeV}$. The production cross section is set to zero if the center-of-mass energy of the incoming partons is below $M_{\mathrm{TH}}$, which therefore provides a bound on the mass of any produced black hole. After the black hole is produced its mass decreases as a result of the emission of Hawking radiation. When the mass of the black hole approaches $M_{\mathrm{D}}$, quantum gravity effects become important. In the final stage of the black hole decay, classical evaporation is no longer a good description and a model is needed to describe the ultimate decay. In such cases where the black hole mass is near $M_{\mathrm{D}}$, the burst model adopted by the BLACKMAX event generator [10] is used in the final part of the decay. No graviton initial-state radiation or emission from the black hole is considered in this paper. Models of rotating and nonrotating black holes are both studied. The track multiplicity is predicted to be slightly lower for rotating black holes [11]. 
A previous result from ATLAS [12] in this final state excludes at $95 \%$ confidence level (C.L.) the production of black holes with $M_{\mathrm{TH}} \leq 3.3,3.6$, and $3.7 \mathrm{TeV}$ for $M_{\mathrm{D}}$ of $1.5 \mathrm{TeV}$ and for $n=2,4$, and 6 , respectively. A previous search by the ATLAS Collaboration in a lepton + jets final state [13] excludes at 95\% C.L. black holes with $M_{\mathrm{TH}} \leq$ $4.5 \mathrm{TeV}$ for $M_{\mathrm{D}}=1.5 \mathrm{TeV}$ and $n=6$. The CMS Collaboration has conducted searches in a multiobject final state and excluded the production of black holes at 95\% C.L. with $M_{\mathrm{TH}} \leq 5.7,6.1$, and $6.2 \mathrm{TeV}$ for $M_{\mathrm{D}}=$ $1.5 \mathrm{TeV}$ and for $n=2,4$, and 6 , respectively $[14,15]$.

The rest of this paper is organized as follows. A brief description of the ATLAS detector is given in Sec. II. The data and simulation samples are described in Sec. III, followed by the event selection in Sec. IV. The background estimation techniques are discussed in Sec. V. The final results and their interpretation are presented in Sec. VI.

\section{THE ATLAS DETECTOR}

The ATLAS detector [16] is a multipurpose detector with a forward-backward symmetric cylindrical geometry covering nearly the entire solid angle [17] around the collision point with layers of tracking detectors, calorimeters, and muon chambers. The inner detector is immersed in a $2 \mathrm{~T}$ axial magnetic field, provided by a solenoid, in the $z$ direction and provides charged particle tracking in the pseudorapidity range $|\eta|<2.5$. A silicon pixel detector covers the luminous region and typically provides three measurements per track, followed by a silicon microstrip tracker (SCT) that provides measurements from eight strip layers. In the region with $|\eta|<2.0$, the silicon detectors are complemented by a transition radiation tracker (TRT), which provides more than 30 straw-tube measurements per track.

The calorimeter system covers the range $|\eta|<4.9$. Lead/liquid argon (lead/LAr) electromagnetic sampling calorimeters cover the range $|\eta|<3.2$, with an additional thin lead/LAr presampler covering $|\eta|<1.8$ to correct for energy loss in material upstream of the calorimeters. Hadronic calorimetry is provided by a steel/scintillator-tile calorimeter over $|\eta|<1.7$ and two copper/LAr endcap calorimeters over $1.75<|\eta|<3.2$. The solid angle coverage is completed with forward copper/LAr and tungsten/ LAr calorimeters for electromagnetic and hadronic measurements, respectively, up to $|\eta|$ of 4.9.

The muon spectrometer consists of separate trigger and high-precision tracking chambers that measure the deflection of muon tracks in a magnetic field with a bending integral in the range of $2 \mathrm{~T} \mathrm{~m}$ to $8 \mathrm{~T} \mathrm{~m}$. The magnetic field is generated by three superconducting air-core toroid magnet systems. The tracking chambers cover the region $|\eta|<2.7$ with three layers of monitored drift tubes supplemented by cathode strip chambers in the innermost region of the endcap muon spectrometer. The muon trigger system covers the range $|\eta|<2.4$ with resistive plate chambers in the barrel, and thin gap chambers in the endcap regions.

\section{DATA AND MONTE CARLO SAMPLES}

The data used in this analysis were collected with the ATLAS detector from proton-proton collisions produced at $\sqrt{s}=8 \mathrm{TeV}$ in 2012 . The data correspond to an integrated luminosity of $20.3 \mathrm{fb}^{-1}$. The uncertainty on the luminosity is $2.8 \%$ and is derived, following the same methodology as that detailed in Ref. [18], from a preliminary calibration of the luminosity scale obtained from beam-separation scans performed in November 2012. The events used for this analysis were recorded with a single-muon trigger with a threshold at $36 \mathrm{GeV}$ on the muon $p_{\mathrm{T}}$. The single-muon trigger efficiency reaches a plateau for muons with $p_{\mathrm{T}}>40 \mathrm{GeV}$ and the plateau efficiency is $71 \%$ in the barrel and $87 \%$ in the endcap for muons reconstructed offline. The inefficiency in the trigger is driven mainly by the uninstrumented regions of the muon trigger system.

Monte Carlo (MC) samples are used for both signal and background modeling. The ATLAS detector is simulated using GEANT [19], and simulation samples [20] are reconstructed using the same software as that used for the collision data. The effect of additional proton-proton collisions in the same or neighboring bunch crossings is modeled by overlaying simulated minimum-bias events onto the original hard-scattering event. MC events are then reweighted so that the reconstructed vertex multiplicity distribution agrees with the one from data.

The dominant background processes are top-quark pair $(t \bar{t})$, diboson, and $W+$ jets production with smaller contributions from single-top production. Background MC samples for the $t \bar{t}$ and the single-top ( $W t$-channel) processes are generated using POWHEG [21] and the CT10 [22] parton distribution functions (PDFs). Fragmentation and hadronization of the events is done with PYTHIA v6.426 [23] using the Perugia tune [24]. The top-quark mass is fixed at $172.5 \mathrm{GeV}$. Alternative samples for studying the systematic uncertainty are made using the ALPGEN v2.14 [25] or MC@TNLO v4.03 [26] generators with HERWIG v6.520 [27] used for hadronization and JIMMY v4.31 [28] used to model the underlying event for both generators. The nominal single-top sample uses the diagram-removal scheme [29] and an alternative sample using the diagramsubtraction scheme is produced for systematic studies. Diboson samples ( $W Z$ and $Z Z$ ) are generated and hadronized using SHERPA v1.4.1 [30]. The diboson samples are produced with the CT10 PDF set and use the ATLAS Underlying Event Tune 2B (AUET2B) [31], which provides a set of parameters that well describes the ATLAS measurements of the additional activity (underlying event) in hard-scattering events. These samples include the case where the $Z$ boson (or $\gamma^{*}$ ) is off shell, with the invariant mass of the $\gamma^{*}$ required to be above twice the muon mass. 
Signal MC samples are generated using BLACKMAX v2.02 [10,32] and the "MSTW 2008 LO" set from the parton distributions given in [33] with the mass of the black hole used as the factorization and renormalization scale. The signal samples are hadronized with PYTHIA v8.165 [34] using the AUET2B tune. Signal samples for rotating and nonrotating black holes are produced by varying $M_{\mathrm{D}}$ between $1.0 \mathrm{TeV}$ and $4.5 \mathrm{TeV}$, and $M_{\mathrm{TH}}$ between $3.0 \mathrm{TeV}$ and $6.5 \mathrm{TeV}$. In each case, samples are generated with $n=2,4$, and 6. As an illustration, the expected yield from rotating black holes in a model with $n=4, M_{\mathrm{TH}}=5 \mathrm{TeV}$, and $M_{\mathrm{D}}=1.5 \mathrm{TeV}$ is shown throughout the paper.

\section{EVENT SELECTION}

Events in data passing the single-muon trigger are selected for this analysis. The detector was required to have been operating properly when these events were collected. Events are also required to have a primary vertex reconstructed from at least five tracks with $p_{\mathrm{T}}>400 \mathrm{MeV}$. In events with multiple vertices, the vertex whose associated tracks have the largest $\Sigma p_{\mathrm{T}}^{2}$ is identified as the primary vertex.

Muon candidates are reconstructed from tracks measured in the muon spectrometer (MS). The MS tracks are matched with inner detector (ID) tracks using a procedure that takes material effects into account. The final parameters for the muon candidates are obtained from a statistical combination of the measured quantities in the MS and the ID. The muon candidates must satisfy $|\eta|<2.4$ and have $p_{\mathrm{T}}>15 \mathrm{GeV}$. The quality of the ID track associated with a muon is ensured by imposing requirements [35] on the number of pixel, SCT, and TRT hits associated with the track. The ID tracks must also pass a requirement on the longitudinal impact parameter $\left(z_{0}\right)$ with respect to the primary vertex, $\left|z_{0} \sin \theta\right|<1.5 \mathrm{~mm}$.

Events are required to have at least two muons. The two muons with the highest $p_{\mathrm{T}}$ are required to have the same charge. The muon with the highest $p_{\mathrm{T}}$ in the event is called the leading muon, while the muon with the second highest $p_{\mathrm{T}}$ is called the subleading muon. The leading muon is required to satisfy $p_{\mathrm{T}}>40 \mathrm{GeV}$ to be above the trigger threshold and pass requirements on isolation and transverse impact parameter as described below. No such requirements are made for the subleading muon.

The muon isolation is constructed from the sum of transverse momenta of other ID tracks in a cone in $\eta-\phi$ space of radius $\Delta R=\sqrt{(\Delta \eta)^{2}+(\Delta \phi)^{2}}=0.2$ around the muon. For the leading muon, the sum is required to be less than $20 \%$ of the muon $p_{\mathrm{T}}$. The impact parameter significance for the muons is defined as $\left|d_{0} / \sigma\left(d_{0}\right)\right|$, where $d_{0}$ is the transverse impact parameter of the muon, and $\sigma\left(d_{0}\right)$ is the associated uncertainty. The leading muon must satisfy $\left|d_{0} / \sigma\left(d_{0}\right)\right| \leq 3$.0. The leading and subleading muons are required to be separated by $\Delta R>0.2$.
The total track multiplicity $\left(N_{\text {trk }}\right)$ of the event is calculated by considering all ID tracks with $p_{\mathrm{T}}>10 \mathrm{GeV}$ and $|\eta|<2.5$ that pass the same quality and $z_{0}$ criteria as those for the muon ID tracks. The track selection is thus less stringent than the muon selection and the track multiplicity counts the two muons as well.

All selections except the trigger requirements are applied to the MC events. The MC events are assigned a weight based on their probability to pass the trigger requirements. The total probability is calculated by considering each muon in the event and the individual probability of the muon to pass the trigger selection. The MC events are also corrected to account for minor differences between data and MC simulation in the muon reconstruction and identification efficiencies by applying $p_{\mathrm{T}^{-}}$and $\eta$-dependent scale factors. The tracking efficiency in MC simulation [36] is consistent with data and has been confirmed with additional studies of tracking performance in a dense environment [37]. Thus no corrections are applied to the simulation for tracking performance.

Signal and validation regions are defined starting from the same basic requirements so that a large ratio of signal to background is expected in the former and very little signal in the latter. The validation region can then be used to ensure that, but comparing expectations with data, the backgrounds are well described, understood, and can be extrapolated into the signal region, after the like-sign dimuon preselection described above and using the $N_{\text {trk }}$ definition. The signal region is defined as follows:

(i) leading-muon $p_{\mathrm{T}}>100 \mathrm{GeV}$, and

(ii) track multiplicity $N_{\text {trk }} \geq 30$.

The validation regions are defined by inverting one or both of the above requirements. Explicitly, the validation regions are split into two types:

(i) Leading muon satisfies $40<p_{\mathrm{T}}<100 \mathrm{GeV}$ without any requirement on $N_{\text {trk }}$.

(ii) Leading-muon $p_{\mathrm{T}}>100 \mathrm{GeV}$ and $N_{\text {trk }}<24$.

By splitting the validation region into subregions, additional validation of the various components making up the background can be made as described in the next section.

\section{BACKGROUND ESTIMATION}

The backgrounds from Standard Model processes are divided into two categories for ease of estimation: processes where the two muons come from correlated decay chains and processes that produce like-sign dimuons in uncorrelated decay chains. Examples of correlated decay chains are the decays of $t \bar{t}$ events, where there is a fixed branching ratio to obtain like-sign dimuons. The most likely scenario in $t \bar{t}$ events is where the leading isolated muon arises from the decay of a $W$ boson from one of the top quarks, and the subleading muon of the same charge comes either from the semileptonic decay of a $b$ quark from the other top quark, or from the sequential decay $b \rightarrow$ $c X \rightarrow \mu X^{\prime}$ of a $b$ quark from the same top quark. 
The uncorrelated background estimates arise predominantly from the $W+$ jets process, where the $W$ boson decay gives rise to the leading isolated muon and the other muon arises from an in-flight $\pi / K$ decay, or the semileptonic decay of a $B$ or $D$ hadron. Processes such as $Z+$ jets, and single top in the $s$ and $t$ channels also give rise to uncorrelated backgrounds when the leading isolated muon arises from the vector boson decay, and one of the jets gives rise to the second muon. The second muon is referred to as a "fake" muon in the subsequent discussion of the uncorrelated background estimate.

\section{A. Correlated background estimates}

The following sources of correlated backgrounds are considered: $t \bar{t}$ production, diboson production, and single-top production in the $W t$ channel. Each of the three correlated backgrounds is estimated from dedicated MC samples. The background from the $W t$ process is small and it has been merged with the $t \bar{t}$ background in the subsequent discussion and presentation. Other possible sources such as $t \bar{t} W$ or $t Z$ production and backgrounds from charge misidentification of muons were found to be negligible.

The sources of uncertainty on the $t \bar{t}$ background are the choice of MC event generator and parton-showering model, the amount of initial- and final-state radiation (ISR/FSR), and the theoretical uncertainty on the production cross section. The $t \bar{t}$ cross section used is $\sigma_{t \bar{t}}=$ $238_{-24}^{+22} \mathrm{pb}$ for a top-quark mass of $172.5 \mathrm{GeV}$. It has been calculated at approximate next-to-next-to-leading order (NNLO) in QCD with HATHOR v1.2 [38] using the MSTW 2008 90\% NNLO PDF sets. It incorporates PDF + $\alpha_{S}$ uncertainties, according to the MSTW prescription [39], added in quadrature with the scale uncertainty and has been cross-checked with the calculation of Cacciari et al. [40] as implemented in TOP++ v1.0 [41]. The uncertainty on the parton-showering model is assessed by comparing the nominal $t \bar{t}$ prediction with a prediction made using a powheg + herwig sample. The generator uncertainty is assessed by comparing the POWHEG prediction with predictions made using the ALPGEN and MC@NLO samples. The ISR/FSR uncertainty is determined by using the ACERMC [42] generator interfaced to PYTHIA, and by varying the ISR and FSR scale $\Lambda_{\mathrm{QCD}}$, as well as the ISR and FSR cutoff scale. The effect of the top-quark mass is studied by generating dedicated samples with top-quark masses of $170 \mathrm{GeV}$ and $175 \mathrm{GeV}$ and is found to be negligible.

The diboson backgrounds have an uncertainty of $6 \%$ on the production cross section [43] and a combined generator and parton showering uncertainty of $24 \%$ based on comparisons between SHERPA and POWHEG, and from renormalization and factorization scale variations [44].

In addition to the uncertainties described above, uncertainties from the measurement of trigger efficiency, the muon reconstruction and identification (including uncertainties due to muon $p_{\mathrm{T}}$ resolution), and the tracking
TABLE I. The systematic uncertainties on the event yields in the signal region for the different backgrounds and sources, in percent. The uncertainties on signal acceptance are also summarized in the table.

\begin{tabular}{lcccc}
\hline \hline Source & $\mu+$ fake & $t \bar{t}$ & Diboson & Signal \\
\hline Fake rate measurement & 34 & & & \\
Photon trigger & 13 & & & \\
Prompt correction & 18 & & & \\
ISR/FSR & & 0.7 & & \\
Parton showering & & 9 & & \\
Generator & 11 & 24 & \\
Cross section & 10 & 6 & \\
Muon trigger & 1.2 & 1.3 & 1.3 \\
Muon reconstruction & & 2.0 & 1.2 & 2.3 \\
Luminosity & & 2.8 & 2.8 & 2.8 \\
Tracking efficiency & & 10 & 10 & 10 \\
Fiducial efficiency & & & & 15 \\
PDF (acceptance) & & & & 5 \\
Total & 41 & 21 & 27 & 19 \\
\hline \hline
\end{tabular}

efficiency are considered for each background along with the uncertainty on the integrated luminosity $(2.8 \%)$. The total systematic uncertainties on the final background estimates from the different sources are summarized in Table I.

\section{B. Uncorrelated background estimates}

The uncorrelated background is estimated from data by first measuring the probability for a track to be reconstructed as a muon in a control sample. This probability is then applied to data events with one muon and at least one track to predict the number of dimuon events. This probability is referred to as a fake rate in the subsequent discussion, and the background estimate is referred to as the $\mu+$ fake background.

The fake rate is measured in a control sample consisting of photon + jet events. These events are collected by a single-photon trigger with a threshold at $40 \mathrm{GeV}$ on the photon transverse momentum. The trigger is prescaled, and the collected data set corresponds to an integrated luminosity of $56 \mathrm{fb}^{-1}$. The photon is required to have $p_{\mathrm{T}}>$ $45 \mathrm{GeV}$, and to satisfy the requirements of Ref. [45]. The photon is also required to satisfy $E_{\mathrm{T}}^{\Delta R \leq 0.4}<5 \mathrm{GeV}$, where $E_{\mathrm{T}}^{\Delta R \leq 0.4}$ is the sum of transverse energies of cells in the electromagnetic and hadronic calorimeters in a cone of 0.4 around the photon axis (excluding the cells associated with the photon). The denominator for the fake rate measurement is the number of events with one photon and at least one track. The track must satisfy all the requirements imposed on an ID track associated with a muon as described in Sec. IV. The track is required to be separated from the photon by $\Delta R>0.4$. The numerator is the subset of these events that have at least one muon passing all the criteria associated with the subleading muon as described 
in Sec. IV. In events with more than one track (muon), the track (muon) with the highest $p_{\mathrm{T}}$ is chosen. The fake rate can have contributions from processes such as $W(\mu \nu) \gamma$ and $Z(\mu \mu) \gamma$ that produce prompt muons and bias the fake rate measurement. This prompt-muon bias is corrected by subtracting these contributions based on MC samples generated using SHERPA. The prompt-muon correction ranges from approximately $1 \%$ at muon $p_{\mathrm{T}}=15 \mathrm{GeV}$ to $30 \%$ at $p_{\mathrm{T}}=100 \mathrm{GeV}$.

The fake rate is parametrized as a function of the $p_{\mathrm{T}}$ and $\eta$ of the track, and as a function of $N_{\text {trk }}$. Since the signal black hole models produce isolated photon events as well, the fake rate is measured by requiring $N_{\text {trk }}<10$ to reduce any potential signal contamination of this control sample. The $N_{\text {trk }}$ dependence is parametrized with a linear fit, and extrapolated for all events with $N_{\text {trk }}>10$. The average fake rate based on the criteria defined here is approximately $1 \%$. The fake rate is consistent with that obtained from photon + jet or $W+$ jet MC samples. The final $\mu+$ fake background estimate is obtained by selecting events with one muon satisfying the requirements of a leading muon, and one track of the same charge satisfying the requirements associated with an ID track. These $\mu^{ \pm}$track $^{ \pm}$events are then assigned a weight based on the fake rate calculated for the track, and are then taken through the rest of the analysis chain in the same way as $\mu^{ \pm} \mu^{ \pm}$events, with the track acting as the proxy for the subleading muon. There is a correction to the fake estimate from overcounting due to $t \bar{t}$ and diboson events populating the $\mu^{ \pm}$track ${ }^{ \pm}$events in data. This correction is estimated from MC simulation to be $2 \%$ and is negligible compared to the systematic uncertainty on the fake estimate.

The uncertainties in the $\mu+$ fake background estimate arise from the statistical uncertainties in measuring the fake rate, the choice of the photon trigger used for the control sample, and the prompt-muon bias correction. The statistical uncertainty in the fake rate is propagated to the final background estimate, along with the uncertainty in the fit parameters used to parametrize the $N_{\text {trk }}$ dependence. The fake rate is remeasured using data collected by a single-photon trigger with a $p_{\mathrm{T}}$ threshold of $80 \mathrm{GeV}$, and the background estimate is recalculated to assess the uncertainty due to the photon trigger. To assess the uncertainty due to the prompt correction, the correction is varied by $\pm 15 \%$ of its nominal value to obtain "up" and "down" fake rates. The final $\mu+$ fake background estimate is calculated with the up and down fake rates, and the larger variation from the nominal estimate is assigned as a systematic uncertainty. The choice of $\pm 15 \%$ is motivated by the uncertainties described in Ref. [46] that include experimental uncertainties on photon reconstruction and identification, and theoretical uncertainties on the production cross sections of the $W \gamma / Z \gamma$ processes. Table I shows the effect of the systematic uncertainties on the final $\mu+$ fake background estimate.

\section{RESULTS AND INTERPRETATION}

The background estimation techniques described in the previous section are tested in the validation regions defined in Sec. IV. Table II shows the predicted backgrounds in the various validation regions and the observed yields. The signal contamination is negligible in all the validation regions. Overall, good agreement is observed between the prediction and the observation.

Figure 1 shows the leading-muon $p_{\mathrm{T}}$ distribution for all like-sign dimuon events (satisfying the preselection). Figure 2 shows the dimuon invariant mass distribution after imposing the $p_{\mathrm{T}}>100 \mathrm{GeV}$ requirement on the leading muon. Figure 3 shows the distribution of the dimuon azimuthal separation, $\Delta \phi_{\mu \mu}$ for events with $N_{\text {trk }} \geq 10$ and where the leading muon has $p_{\mathrm{T}}>100 \mathrm{GeV}$. Figure 4 shows the track multiplicity distribution, which shows good agreement between predicted backgrounds and data. The predicted background and the observed data events in the signal region are shown in Table III. The figures and the table show the expected signal contribution from rotating black holes in a model with $n=4, M_{\mathrm{TH}}=5 \mathrm{TeV}$, and $M_{\mathrm{D}}=1.5 \mathrm{TeV}$.

TABLE II. The predicted backgrounds in the validation regions compared to the number of observed data events. The uncertainties shown on the total background are the combined statistical and systematic uncertainties.

\begin{tabular}{|c|c|c|c|c|c|}
\hline$N_{\text {trk }}$ & $t \bar{t}$ & Diboson & $\mu+$ fake & Total & Data \\
\hline \multicolumn{6}{|c|}{$40 \mathrm{GeV}<$ Leading-muon $p_{\mathrm{T}}<100 \mathrm{GeV}$} \\
\hline$N_{\text {trk }}<10$ & 10000 & 800 & 20000 & $31000 \pm 4000$ & 28988 \\
\hline $10 \leq N_{\text {trk }}<20$ & 800 & 3 & 400 & $1200 \pm 100$ & 1103 \\
\hline$N_{\text {trk }} \geq 20$ & 16 & 0.1 & 6.8 & $23 \pm 3$ & 12 \\
\hline \multicolumn{6}{|c|}{ Leading-muon $p_{\mathrm{T}} \geq 100 \mathrm{GeV}$} \\
\hline$N_{\text {trk }}<10$ & 2400 & 140 & 2300 & $4800 \pm 600$ & 4428 \\
\hline $10 \leq N_{\text {trk }} \leq 11$ & 190 & 3 & 76 & $270 \pm 31$ & 271 \\
\hline $12 \leq N_{\text {trk }} \leq 14$ & 133 & 1.1 & 42 & $176 \pm 21$ & 167 \\
\hline $15 \leq N_{\text {trk }} \leq 19$ & 60 & 0.3 & 17 & $77 \pm 9$ & 68 \\
\hline $20 \leq N_{\text {trk }} \leq 24$ & 10 & 0.1 & 2.9 & $13 \pm 2$ & 13 \\
\hline
\end{tabular}




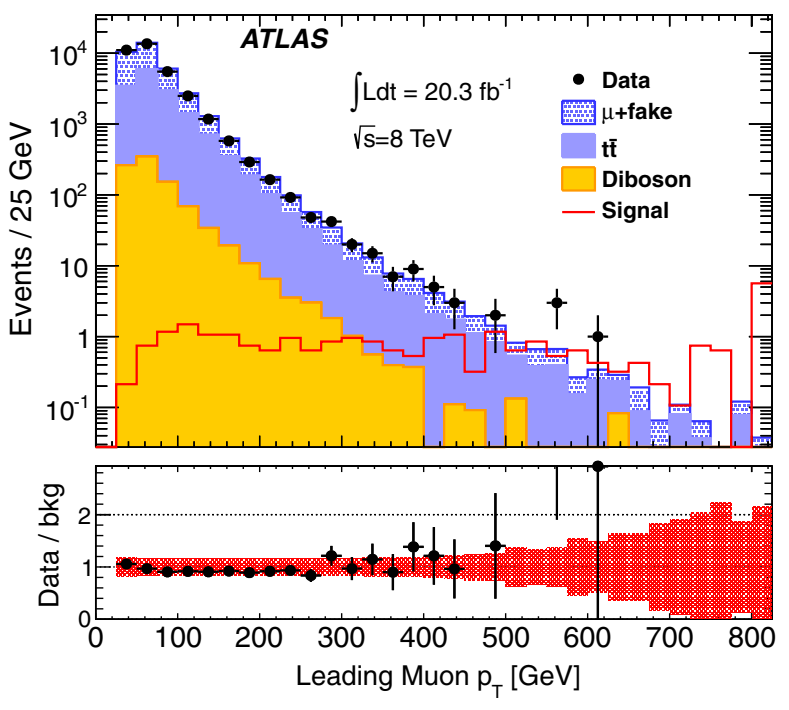

FIG. 1 (color online). The leading-muon $p_{\mathrm{T}}$ distribution for the predicted background and observed data for all like-sign dimuon events passing the preselection criteria. The background histograms are stacked. The signal histogram is overlaid. The last bin along the $x$ axis shows the overflows. The bottom panel shows the ratio of data to the expected background (points) and the total uncertainty on the background (shaded area).
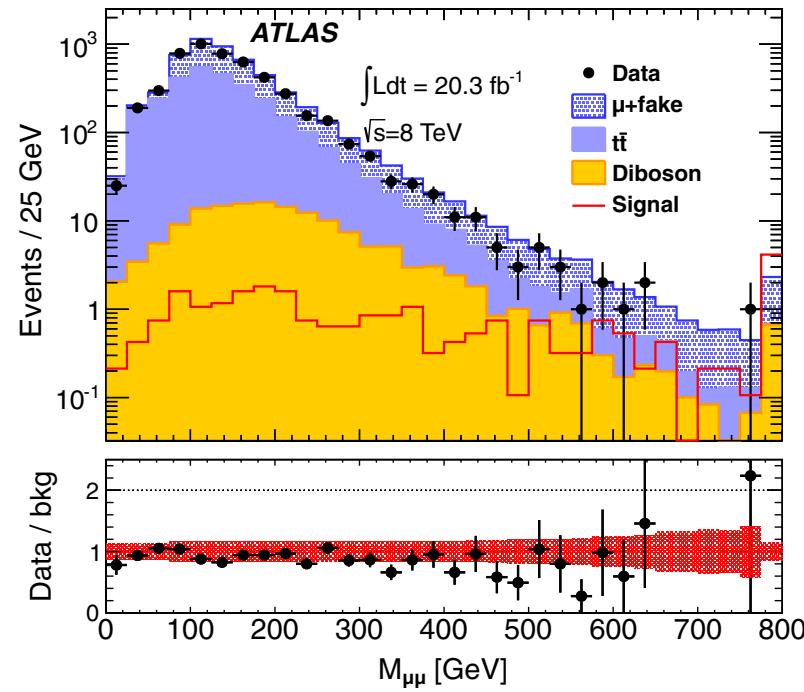

FIG. 2 (color online). The dimuon invariant mass $\left(\mathrm{M}_{\mu \mu}\right)$ distribution for the predicted background and observed data for like-sign dimuon events where the leading muon satisfies $p_{\mathrm{T}}>$ $100 \mathrm{GeV}$. The background histograms are stacked. The signal histogram is overlaid. The last bin along the $x$ axis shows the overflows. The bottom panel shows the ratio of data to the expected background (points) and the total uncertainty on the background (shaded area).

No events are observed in the signal region, which is consistent with the Standard Model prediction. This result is used to set upper limits on the number of events from non-Standard Model sources. The $C L_{s}$ method [47] is used

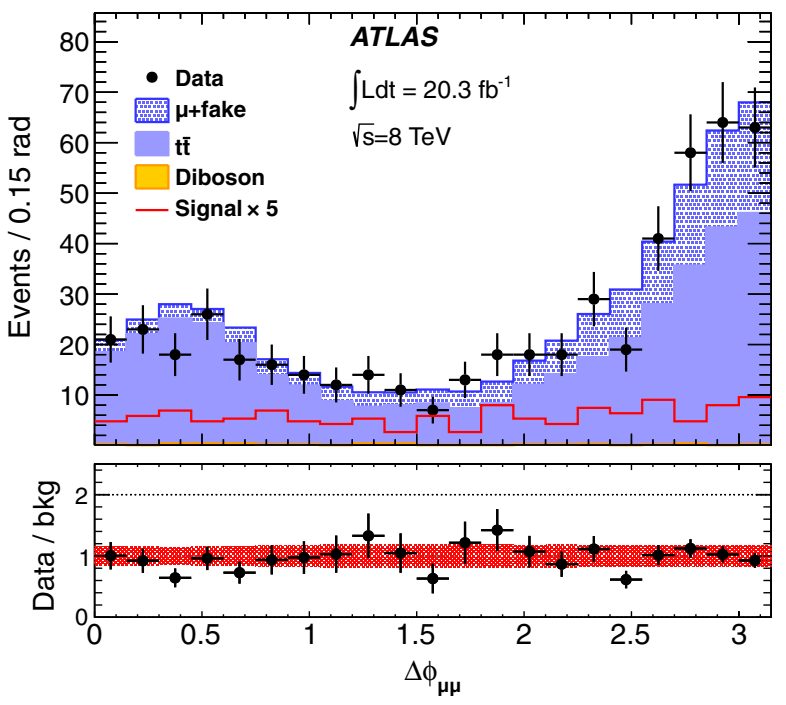

FIG. 3 (color online). The dimuon azimuthal separation $\left(\Delta \phi_{\mu \mu}\right)$ distribution for the predicted background and observed data for like-sign dimuon events where the leading muon satisfies $p_{\mathrm{T}}>100 \mathrm{GeV}$, and with $N_{\text {trk }} \geq 10$. The background histograms are stacked. The signal histogram is overlaid. The bottom panel shows the ratio of data to the expected background (points) and the total uncertainty on the background (shaded area).

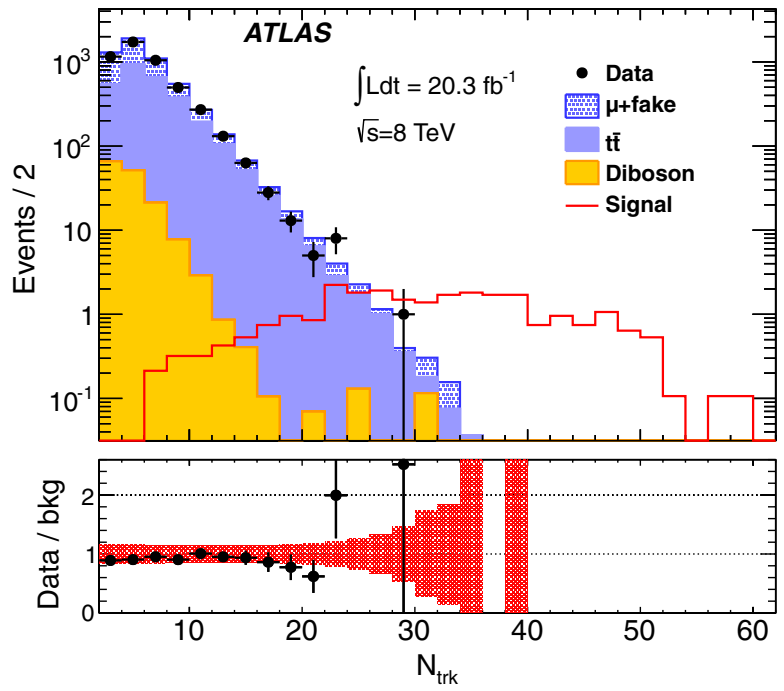

FIG. 4 (color online). The track multiplicity (N-trk) distribution $\left(p_{\mathrm{T}}^{\mathrm{trk}}>10 \mathrm{GeV}\right.$ ) for the predicted background and observed data for events where the leading muon has $p_{\mathrm{T}}>100 \mathrm{GeV}$. The background histograms are stacked. The signal histogram is overlaid. The bottom panel shows the ratio of data to the expected background (points) and the total uncertainty on the background (shaded area).

to calculate $95 \%$ C.L. upper limits on $\sigma_{\text {vis }}=\sigma \times \mathrm{BR} \times$ $A \times \epsilon$, where $\sigma_{\text {vis }}$ is the visible cross section, $\sigma$ is the total cross section, $\mathrm{BR}$ is the inclusive branching ratio to likesign dimuons, $A$ is the acceptance, and $\epsilon$ is the reconstruction efficiency for non-Standard Model contributions in 
TABLE III. The predicted background in the signal region compared to the number of observed events in data. The MC predictions are shown together with statistical and systematic uncertainties. The expected signal contribution from rotating black holes in a model with $n=4, M_{\mathrm{TH}}=5 \mathrm{TeV}$, and $M_{\mathrm{D}}=$ $1.5 \mathrm{TeV}$ is also shown.

\begin{tabular}{lc}
\hline \hline Source & Signal region \\
\hline$\mu+$ fake & $0.21 \pm 0.09 \pm 0.09$ \\
$t \bar{t}$ & $0.22 \pm 0.08 \pm 0.04$ \\
Diboson & $0.12 \pm 0.08 \pm 0.03$ \\
Total & $0.55 \pm 0.15 \pm 0.10$ \\
Data & 0 \\
Signal & $14.2 \pm 1.3 \pm 2.7$ \\
\hline \hline
\end{tabular}

this final state in the signal region. The observed 95\% C.L. limit on $\sigma_{\text {vis }}$ is $0.16 \mathrm{fb}$. The observed limit agrees well with the expected limit of $0.16 \mathrm{fb}$. The standard deviation $(\sigma)$ bands on the expected limit at $1 \sigma$ and $2 \sigma$ are $0.15-0.22 \mathrm{fb}$ and $0.15-0.29 \mathrm{fb}$, respectively.

Exclusion contours in the plane defined by $M_{\mathrm{TH}}$ and $M_{\mathrm{D}}$ for rotating and nonrotating black holes for $n=2$, 4, and 6 are obtained. No theoretical uncertainty on the signal prediction is assessed, i.e. the exclusion limits are set for the exact benchmark models as described in Sec. III.

The signal acceptance is measured from the event generator (truth) by imposing the following selections at the particle level. Each event must have at least two true muons with $p_{\mathrm{T}}>15 \mathrm{GeV}$ and $|\eta|<2.4$, and the leading two muons in $p_{\mathrm{T}}$ must have the same charge. The leading muon must satisfy $p_{\mathrm{T}}>100 \mathrm{GeV}$. The leading-muon truth isolation $\left(I_{\mathrm{gen}}\right)$ is defined as the sum of $p_{\mathrm{T}}$ of all charged particles with $p_{\mathrm{T}}>1 \mathrm{GeV}$ within a cone of $\Delta R=0.2$ around the muon (excluding the muon). The leading muon is required to satisfy $I_{\text {gen }}<0.25 \times p_{\mathrm{T}}$. Each event must also have at least 30 charged particles satisfying $p_{\mathrm{T}}>$ $10 \mathrm{GeV}$ and $|\eta|<2.5$. The ratio of events passing these selections at particle level to the total number of generated events gives the acceptance. The acceptance varies from $11 \%$ to $0.2 \%$ across the range of model parameters considered here.

The acceptance is then corrected to take into account detector effects. The correction factor, $\epsilon_{\mathrm{fid}}$, is defined as the ratio of number of events passing the selection criteria after full detector reconstruction to the number of events passing the acceptance criteria at the particle level. The factor is found to be independent of the number of extra dimensions, and is linearly dependent on $k=M_{\mathrm{TH}} / M_{\mathrm{D}}$. The linear dependence is assessed separately for rotating and nonrotating black holes by a fit to the efficiency as a function of $k$. For rotating (nonrotating) signals $\epsilon_{\text {fid }}$ rises from $0.35(0.3)$ for $k=1$ to $0.55(0.65)$ for $k=3$.

The uncertainty on the signal prediction has the following components: the uncertainty on the $\epsilon_{\text {fid }}$ fit parameters, the uncertainty on luminosity, the uncertainty on acceptance due to the PDFs, the experimental uncertainty on acceptance due to muon trigger and identification efficiencies, and the uncertainty due to tracking efficiency. The uncertainty on acceptance due to PDF was estimated by using the 40 error sets associated to the MSTW 2008 LO PDF set. In the signal region, at high $N_{\text {trk }}$, it is possible for small differences between the track reconstruction efficiencies in data and simulation to be magnified. The effect of any possible disagreement between data and simulation is studied by artificially increasing the disagreement and probing the subsequent effect on the signal acceptance. A disagreement of $2 \%$ in the per-track reconstruction efficiency translates to a 5\% uncertainty in the signal acceptance for $N_{\text {trk }} \geq 30$. As a conservative choice, a $10 \%$ uncertainty on signal acceptance is assigned to account for possible disagreements in data and simulation track reconstruction efficiency. The uncertainties are summarized in Table I.

Figure 5 shows the expected and observed exclusion contours for nonrotating black holes for $n=2$, 4, and 6 . Figure 6 shows the same for rotating black holes. In both figures, the $1 \sigma$ uncertainty band on the expected limit is shown for $n=2$. For each value of $n$, the observed limit lies within the $1 \sigma$ band. Lines of constant slope $(k=$ $\left.M_{\mathrm{TH}} / M_{\mathrm{D}}\right)$ of $2,3,4$, and 5 are also shown. The semiclassical approximations used for black hole production and decay are expected to be valid only for large slopes. The effect of choosing a different set of PDFs for signal generation has been studied by considering the CT10 PDF set. The predicted cross sections with the CT10 PDF set are approximately $20 \%$ higher, but this has a negligible impact on the exclusion contours due to the rapidly falling cross section with mass for black hole production.

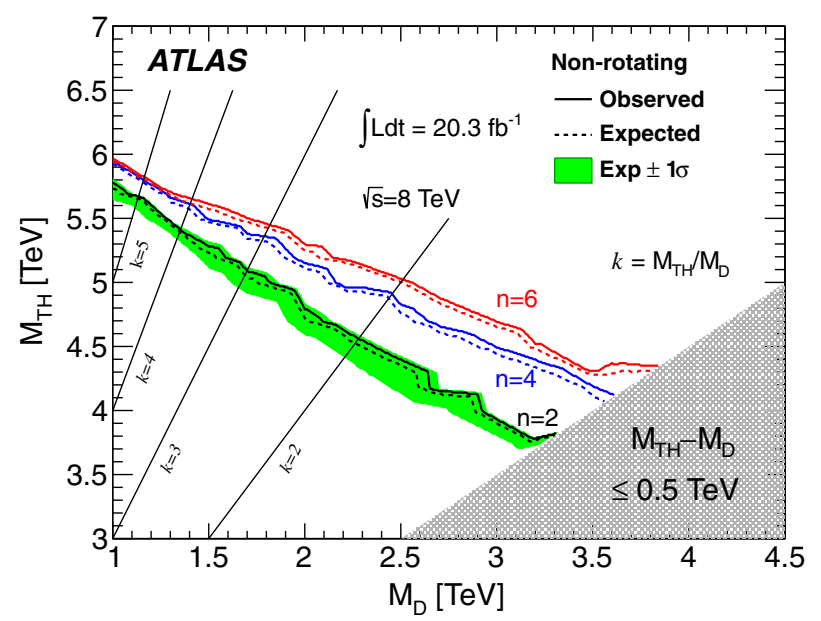

FIG. 5 (color online). The 95\% C.L. exclusion contours for nonrotating black holes in models with $n=2,4$, and 6 . The dashed lines show the expected exclusion contour, the solid lines show the observed exclusion contour. The regions below the contours are excluded by this analysis. The $1 \sigma$ uncertainty on the expected limit for $n=2$ is shown as a band. Lines of constant slope $k=M_{\mathrm{TH}} / M_{\mathrm{D}}=2,3,4$, and 5 are also shown. Only slopes $k \gg 1$ correspond to physical models. 


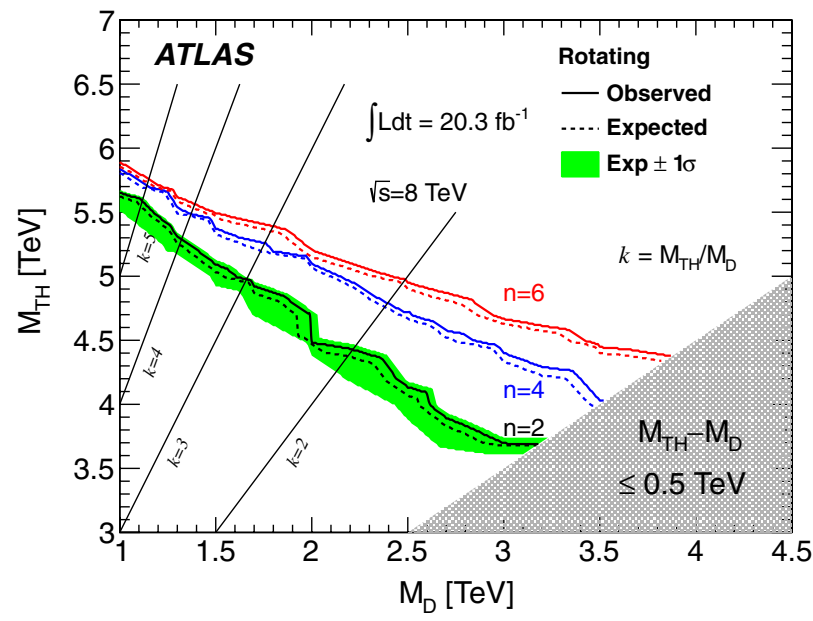

FIG. 6 (color online). The 95\% C.L. exclusion contours for rotating black holes in models with $n=2,4$, and 6 . The dashed lines show the expected exclusion contour, the solid lines show the observed exclusion contour. The regions below the contours are excluded by this analysis. The $1 \sigma$ uncertainty on the expected limit for $n=2$ is shown as a band. Lines of constant slope $k=M_{\mathrm{TH}} / M_{\mathrm{D}}=2,3,4$, and 5 are also shown. Only slopes $k \gg 1$ correspond to physical models.

The theory of large extra dimensions can be embedded into weakly coupled string theory $[48,49]$, giving rise to string balls whose decay would be experimentally similar to the decay of black holes. Models of string balls have two additional parameters $M_{S}$ and $g_{s}$, the string scale and the string coupling constant, respectively, in addition to $M_{\mathrm{TH}}$, $M_{\mathrm{D}}$, and $n$. BLACKMAX is used to simulate the production

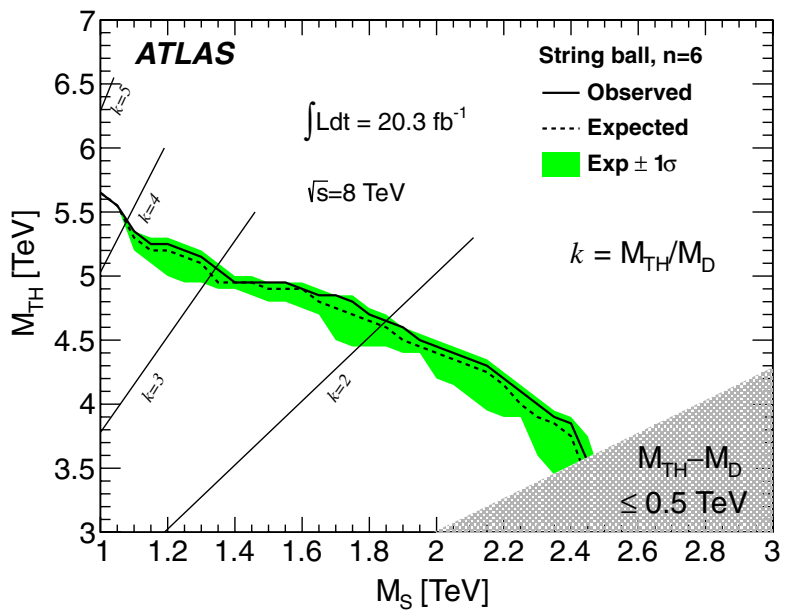

FIG. 7 (color online). The 95\% C.L. exclusion contours for string balls in models with $n=6$. The dashed line shows the expected exclusion contour with the $1 \sigma$ uncertainty shown as a band. The solid line shows the observed exclusion contour. The region below the contour is excluded by this analysis. Lines of constant $k=M_{\mathrm{TH}} / M_{\mathrm{D}}=2,3,4$, and 5 are also shown. Only the values $k \gg 1$ correspond to physical models.
TABLE IV. The lower limits on $M_{\mathrm{TH}}$ at $95 \%$ C.L. are summarized for different models. $M_{\mathrm{D}}$ is fixed at $1.5 \mathrm{TeV}$. For the string ball model, $g_{s}=0.40$ and $M_{S}=M_{\mathrm{D}} / 1.26=1.2 \mathrm{TeV}$.

\begin{tabular}{lcc}
\hline \hline Model & $n$ & $M_{\mathrm{TH}}[\mathrm{TeV}] \geq$ \\
\hline Nonrotating black hole & 2 & 5.3 \\
Nonrotating black hole & 4 & 5.6 \\
Nonrotating black hole & 6 & 5.7 \\
Rotating black hole & 2 & 5.1 \\
Rotating black hole & 4 & 5.4 \\
Rotating black hole & 6 & 5.5 \\
String ball & 6 & 5.3 \\
\hline \hline
\end{tabular}

and decay of string balls, and to obtain exclusion contours in the plane defined by $M_{\mathrm{TH}}$ and $M_{\mathrm{S}}$. Following Ref. [49], the values of $g_{s}$ and $M_{\mathrm{D}}$ are set by $g_{s}^{2}=1 / 5^{\frac{n+2}{n+1}}$, and $M_{\mathrm{D}}=$ $5^{\frac{1}{n+1}} M_{\mathrm{S}}$. The exclusion contour in the $M_{\mathrm{TH}}-M_{\mathrm{S}}$ plane for string balls is shown in Fig. 7 for models with $n=6$ (where $g_{s}=0.40$ and $M_{\mathrm{D}}=1.26 M_{\mathrm{S}}$ ). Table IV shows the summary of lower limits placed on the mass of microscopic black holes and string balls for $M_{\mathrm{D}}=1.5 \mathrm{TeV}$ for different values of $n$.

\section{CONCLUSIONS}

A search for microscopic black holes has been carried out using $20.3 \mathrm{fb}^{-1}$ of data collected by the ATLAS detector in $8 \mathrm{TeV}$ proton-proton collisions at the LHC. No excess of events over the Standard Model background expectations is observed in the final state with a like-sign dimuon pair and high track multiplicity. Exclusion contours in the plane of the fundamental Planck scale $M_{\mathrm{D}}$ and the threshold mass $M_{\mathrm{TH}}$ of black holes are shown and a limit of $0.16 \mathrm{fb}$ at $95 \%$ C.L. is set on the visible cross section for any new physics in the signal region defined by a like-sign dimuon pair and high track multiplicity selection.

\section{ACKNOWLEDGMENTS}

We thank CERN for the very successful operation of the LHC, as well as the support staff from our institutions without whom ATLAS could not be operated efficiently. We acknowledge the support of ANPCyT, Argentina; YerPhI, Armenia; ARC, Australia; BMWF and FWF, Austria; ANAS, Azerbaijan; SSTC, Belarus; CNPq and FAPESP, Brazil; NSERC, NRC, and CFI, Canada; CERN; CONICYT, Chile; CAS, MOST, and NSFC, China; COLCIENCIAS, Colombia; MSMT CR, MPO CR, and VSC CR, Czech Republic; DNRF, DNSRC, and Lundbeck Foundation, Denmark; EPLANET, ERC, and NSRF, European Union; IN2P3-CNRS, CEA-DSM/ IRFU, France; GNSF, Georgia; BMBF, DFG, HGF, MPG, and AvH Foundation, Germany; GSRT and NSRF, Greece; ISF, MINERVA, GIF, DIP, and Benoziyo Center, Israel; INFN, Italy; MEXT and JSPS, Japan; CNRST, Morocco; FOM and NWO, Netherlands; BRF and RCN, 
Norway; MNiSW, Poland; GRICES and FCT, Portugal; MERYS (MECTS), Romania; MES of Russia and ROSATOM, Russian Federation; JINR; MSTD, Serbia; MSSR, Slovakia; ARRS and MIZŠ, Slovenia; DST/NRF, South Africa; MICINN, Spain; SRC and Wallenberg Foundation, Sweden; SER, SNSF, and Cantons of Bern and Geneva, Switzerland; NSC, Taiwan; TAEK, Turkey; STFC, the Royal Society and Leverhulme Trust, United
Kingdom; DOE and NSF, United States of America. The crucial computing support from all WLCG partners is acknowledged gratefully, in particular, from CERN and the ATLAS Tier-1 facilities at TRIUMF (Canada), NDGF (Denmark, Norway, Sweden), CC-IN2P3 (France), KIT/ GridKA (Germany), INFN-CNAF (Italy), NL-T1 (Netherlands), PIC (Spain), ASGC (Taiwan), RAL (UK), and BNL (USA) and in the Tier-2 facilities worldwide.
[1] N. Arkani-Hamed, S. Dimopoulos, and G. R. Dvali, Phys. Lett. B 429, 263 (1998)

[2] I. Antoniadis, N. Arkani-Hamed, S. Dimopoulos, and G. R. Dvali, Phys. Lett. B 436, 257 (1998).

[3] N. Arkani-Hamed, S. Dimopoulos, and G. R. Dvali, Phys. Rev. D 59, 086004 (1999).

[4] P. C. Argyres, S. Dimopoulos, and J. March-Russell, Phys. Lett. B 441, 96 (1998).

[5] T. Banks and W. Fischler, arXiv:hep-th/9906038.

[6] S. Dimopoulos and G. L. Landsberg, Phys. Rev. Lett. 87, 161602 (2001).

[7] S. B. Giddings and S. D. Thomas, Phys. Rev. D 65, 056010 (2002).

[8] P. Kanti, Int. J. Mod. Phys. A 19, 4899 (2004).

[9] S. Hawking, Commun. Math. Phys. 43, 199 (1975).

[10] D.-C. Dai, G. Starkman, D. Stojkovic, C. Issever, E. Rizvi, and J. Tseng, Phys. Rev. D 77, 076007 (2008).

[11] J. A. Frost, J. R. Gaunt, M. O. Sampaio, M. Casals, S. R. Dolan, M. A. Parker, and B. R. Webber, J. High Energy Phys. 10 (2009) 014.

[12] ATLAS Collaboration, Phys. Lett. B 709, 322 (2012).

[13] ATLAS Collaboration, Phys. Lett. B 716, 122 (2012).

[14] CMS Collaboration, J. High Energy Phys. 07 (2013) 178.

[15] CMS Collaboration, J. High Energy Phys. 04 (2012) 061.

[16] ATLAS Collaboration, JINST 3, S08003 (2008).

[17] ATLAS uses a right-handed coordinate system with its origin at the nominal interaction point (IP) in the center of the detector and the $z$ axis along the beam pipe. The $x$ axis points from the IP to the center of the LHC ring, and the $y$ axis points upward. Cylindrical coordinates $(r, \phi)$ are used in the transverse plane, $\phi$ being the azimuthal angle around the beam pipe. The pseudorapidity is defined in terms of the polar angle $\theta$ as $\eta=-\ln \tan (\theta / 2)$.

[18] ATLAS Collaboration, Eur. Phys. J. C 73, 2518 (2013).

[19] S. Agostinelli et al., Nucl. Instrum. Methods Phys. Res. 506, 250 (2003).

[20] ATLAS Collaboration, Eur. Phys. J. C 70, 823 (2010).

[21] T. Melia, P. Nason, R. Rontsch, and G. Zanderighi, J. High Energy Phys. 11 (2011) 078.

[22] H.-L. Lai, M. Guzzi, J. Huston, Z. Li, P. M. Nadolsky, J. Pumplin, and C.-P. Yuan, Phys. Rev. D 82, 074024 (2010).

[23] T. Sjöstrand, P. Edén, C. Friberg, L. Lönnblad, G. Miu, S. Mrenna, and E. Norrbin, Comput. Phys. Commun. 135, 238 (2001).

[24] P.Z. Skands, Phys. Rev. D 82, 074018 (2010).
[25] M. L. Mangano, M. Moretti, F. Piccinini, R. Pittau, and A. D. Polosa, J. High Energy Phys. 07 (2003) 001.

[26] S. Frixione and B. R. Webber, J. High Energy Phys. 06 (2002) 029.

[27] G. Corcella, I. Knowles, G. Marchesini, S. Moretti, K. Odagiri, P. Richardson, M. H. Seymour, and B. R. Webber, J. High Energy Phys. 01 (2001) 010.

[28] J. Butterworth, J. R. Forshaw, and M. Seymour, Z. Phys. C 72, 637 (1996).

[29] S. Frixione, E. Laenen, P. Motylinski, B. R. Webber, and C. D. White, J. High Energy Phys. 07 (2008) 029.

[30] T. Gleisberg, S. Hoeche, F. Krauss, M. Schönherr, S. Schumann, F. Siegert, and J. Winter, J. High Energy Phys. 02 (2009) 007.

[31] ATLAS Collaboration, Report No. ATL-PHYS-PUB2011-009, 2011, http://cds.cern.ch/record/1363300.

[32] D.-C. Dai, C. Issever, E. Rizvi, G. Starkman, D. Stojkovic, and J. Tseng, arXiv:0902.3577.

[33] A. Martin, W. Stirling, R. Thorne, and G. Watt, Eur. Phys. J. C 63, 189 (2009).

[34] T. Sjöstrand, S. Mrenna, and P.Z. Skands, Comput. Phys. Commun. 178, 852 (2008).

[35] ATLAS Collaboration, Report No. ATLAS-CONF-2011063, 2011, http://cds.cern.ch/record/1345743.

[36] ATLAS Collaboration, New J. Phys. 13, 053033 (2011).

[37] ATLAS Collaboration, Eur. Phys. J. C 71, 1795 (2011).

[38] M. Aliev, H. Lacker, U. Langenfeld, S. Moch, P. Uwer, and M. Wiedermann, Comput. Phys. Commun. 182, 1034 (2011).

[39] A. D. Martin, W. J. Stirling, R. S. Thorne, and G. Watt, Eur. Phys. J. C 64, 653 (2009).

[40] M. Cacciari, M. Czakon, M. Mangano, A. Mitov, and P. Nason, Phys. Lett. B 710, 612 (2012).

[41] M. Czakon and A. Mitov, arXiv:1112.5675.

[42] B.P. Kersevan and E. Richter-Was, arXiv:hep-ph/ 0405247.

[43] J. M. Campbell, R. K. Ellis, and C. Williams, J. High Energy Phys. 07 (2011) 018.

[44] ATLAS Collaboration, Phys. Rev. D 87, 052002 (2013).

[45] ATLAS Collaboration, Phys. Rev. D 85, 092014 (2012).

[46] ATLAS Collaboration, Phys. Rev. D 87, 112003 (2013).

[47] A. L. Read, J. Phys. G 28, 2693 (2002).

[48] S. Dimopoulos and R. Emparan, Phys. Lett. B 526, 393 (2002).

[49] D. M. Gingrich and K. Martell, Phys. Rev. D 78, 115009 (2008). 
G. Aad, ${ }^{48}$ T. Abajyan, ${ }^{21}$ B. Abbott,,${ }^{112}$ J. Abdallah, ${ }^{12}$ S. Abdel Khalek, ${ }^{116}$ O. Abdinov, ${ }^{11}$ R. Aben,,${ }^{106}$ B. Abi, ${ }^{113}$ M. Abolins, ${ }^{89}$ O. S. AbouZeid, ${ }^{159}$ H. Abramowicz, ${ }^{154}$ H. Abreu, ${ }^{137}$ Y. Abulaiti, ${ }^{147 a, 147 b}$ B. S. Acharya, ${ }^{165 a, 165 b, b}$ L. Adamczyk, ${ }^{38 a}$ D. L. Adams, ${ }^{25}$ T. N. Addy,${ }^{56}$ J. Adelman, ${ }^{177}$ S. Adomeit, ${ }^{99}$ T. Adye, ${ }^{130}$ S. Aefsky, ${ }^{23}$ T. Agatonovic-Jovin, ${ }^{13 \mathrm{~b}}$ J. A. Aguilar-Saavedra, ${ }^{125 \mathrm{~b}, \mathrm{c}}$ M. Agustoni, ${ }^{17}$ S. P. Ahlen, ${ }^{22}$ A. Ahmad,${ }^{149}$ F. Ahmadov, ${ }^{64, \mathrm{~d}}$ M. Ahsan, ${ }^{41}$ G. Aielli, ${ }^{134 a, 134 b}$ T. P. A. Åkesson, ${ }^{80}$ G. Akimoto, ${ }^{156}$ A. V. Akimov, ${ }^{95}$ M. A. Alam, ${ }^{76}$ J. Albert, ${ }^{170}$ S. Albrand,${ }^{55}$ M. J. Alconada Verzini,${ }^{70}$ M. Aleksa ${ }^{30}$ I. N. Aleksandrov, ${ }^{64}$ F. Alessandria, ${ }^{90 a}$ C. Alexa, ${ }^{26 a}$ G. Alexander, ${ }^{154}$ G. Alexandre, ${ }^{49}$ T. Alexopoulos, ${ }^{10}$ M. Alhroob,${ }^{165 a, 165 c}$ M. Aliev, ${ }^{16}$ G. Alimonti,,${ }^{90 a}$ L. Alio, ${ }^{84}$ J. Alison, ${ }^{31}$ B. M. M. Allbrooke, ${ }^{18}$ L. J. Allison, ${ }^{71}$ P. P. Allport, ${ }^{73}$ S. E. Allwood-Spiers, ${ }^{53}$ J. Almond, ${ }^{83}$ A. Aloisio, ${ }^{103 a, 103 \mathrm{~b}}$ R. Alon, ${ }^{173}$ A. Alonso, ${ }^{36}$ F. Alonso, ${ }^{70}$ A. Altheimer, ${ }^{35}$ B. Alvarez Gonzalez, ${ }^{89}$ M. G. Alviggi, ${ }^{103 a, 103 b}$ K. Amako, ${ }^{65}$ Y. Amaral Coutinho, ${ }^{24 a}$ C. Amelung, ${ }^{23}$ V. V. Ammosov, ${ }^{129, a}$ S. P. Amor Dos Santos, ${ }^{125 a}$ A. Amorim, ${ }^{125 a, e}$ S. Amoroso, ${ }^{48}$ N. Amram, ${ }^{154}$ C. Anastopoulos, ${ }^{30}$ L. S. Ancu, ${ }^{17}$ N. Andari, ${ }^{30}$ T. Andeen, ${ }^{35}$ C. F. Anders, ${ }^{58 b}$ G. Anders, ${ }^{58 a}$ K. J. Anderson, ${ }^{31}$ A. Andreazza, ${ }^{90 a, 90 b}$ V. Andrei, ${ }^{58 a}$ X. S. Anduaga ${ }^{70}$ S. Angelidakis, ${ }^{9}$ P. Anger ${ }^{44}$ A. Angerami, ${ }^{35}$ F. Anghinolfi,,${ }^{30}$ A. V. Anisenkov, ${ }^{108}$ N. Anjos, ${ }^{125 a}$ A. Annovi, ${ }^{47}$ A. Antonaki, ${ }^{9}$ M. Antonelli, ${ }^{47}$ A. Antonov, ${ }^{97}$ J. Antos, ${ }^{145 b}$ F. Anulli, ${ }^{133 a}$ M. Aoki, ${ }^{102}$ L. Aperio Bella, ${ }^{18}$ R. Apolle, ${ }^{119, f}$ G. Arabidze,${ }^{89}$ I. Aracena,${ }^{144}$ Y. Arai,${ }^{65}$ A. T. H. Arce, ${ }^{45}$ S. Arfaoui, ${ }^{149}$ J-F. Arguin, ${ }^{94}$ S. Argyropoulos ${ }^{42}$ E. Arik, ${ }^{19 a, a}$ M. Arik, ${ }^{19 a}$ A. J. Armbruster,${ }^{88}$ O. Arnaez,${ }^{82}$ V. Arnal, ${ }^{81}$ O. Arslan, ${ }^{21}$ A. Artamonov, ${ }^{96}$ G. Artoni, ${ }^{133 a, 133 b}$ S. Asai ${ }^{156}$ N. Asbah, ${ }^{94}$ S. Ask, ${ }^{28}$ B. Asman,,${ }^{147 a, 147 b}$ L. Asquith, ${ }^{6}$ K. Assamagan ${ }^{25}$ R. Astalos, ${ }^{145 a}$ A. Astbury, ${ }^{170}$ M. Atkinson, ${ }^{166}$ N. B. Atlay,${ }^{142}$ B. Auerbach, ${ }^{6}$ E. Auge,${ }^{116}$ K. Augsten, ${ }^{127}$ M. Aurousseau, ${ }^{146 b}$ G. Avolio, ${ }^{30}$ D. Axen, ${ }^{169}$ G. Azuelos, ${ }^{94, g}$ Y. Azuma, ${ }^{156}$ M. A. Baak, ${ }^{30}$ C. Bacci, ${ }^{135 a, 135 b}$ A. M. Bach, ${ }^{15}$ H. Bachacou, ${ }^{137}$ K. Bachas, ${ }^{155}$ M. Backes,${ }^{30}$ M. Backhaus, ${ }^{21}$ J. Backus Mayes,${ }^{144}$ E. Badescu, ${ }^{26 a}$ P. Bagiacchi, ${ }^{133 a, 133 b}$ P. Bagnaia, ${ }^{133 a, 133 b}$ Y. Bai, ${ }^{33 a}$ D. C. Bailey, ${ }^{159}$ T. Bain, ${ }^{35}$ J. T. Baines, ${ }^{130}$ O. K. Baker, ${ }^{177}$ S. Baker,${ }^{77}$ P. Balek, ${ }^{128}$ F. Balli, ${ }^{137}$ E. Banas,${ }^{39}$ Sw. Banerjee, ${ }^{174}$ D. Banfi, ${ }^{30}$ A. Bangert, ${ }^{151}$ V. Bansal, ${ }^{170}$ H. S. Bansil, ${ }^{18}$ L. Barak,${ }^{173}$ S. P. Baranov, ${ }^{95}$ T. Barber, ${ }^{48}$ E. L. Barberio, ${ }^{87}$ D. Barberis, ${ }^{50,50 b}$ M. Barbero, ${ }^{84}$ D. Y. Bardin, ${ }^{64}$ T. Barillari, ${ }^{100}$ M. Barisonzi, ${ }^{176}$ T. Barklow, ${ }^{144}$ N. Barlow, ${ }^{28}$ B. M. Barnett,,${ }^{130}$ R. M. Barnett, ${ }^{15}$ A. Baroncelli, ${ }^{135 a}$ G. Barone ${ }^{49}$ A. J. Barr, ${ }^{119}$ F. Barreiro, ${ }^{81}$ J. Barreiro Guimarães da Costa, ${ }^{57}$ R. Bartoldus, ${ }^{144}$ A. E. Barton, ${ }^{71}$ V. Bartsch, ${ }^{150}$ A. Bassalat, ${ }^{116}$ A. Basye, ${ }^{166}$ R. L. Bates,${ }^{53}$ L. Batkova, ${ }^{145 a}$ J. R. Batley, ${ }^{28}$ M. Battistin, ${ }^{30}$ F. Bauer,${ }^{137}$ H. S. Bawa, ${ }^{144, h}$ S. Beale, ${ }^{99}$ T. Beau, ${ }^{79}$ P. H. Beauchemin,${ }^{162}$ R. Beccherle, ${ }^{50 a}$ P. Bechtle ${ }^{21}$ H. P. Beck,${ }^{17}$ K. Becker, ${ }^{176}$ S. Becker, ${ }^{99}$ M. Beckingham, ${ }^{139}$ A. J. Beddall, ${ }^{19 c}$ A. Beddall, ${ }^{19 \mathrm{c}}$ S. Bedikian, ${ }^{177}$ V. A. Bednyakov, ${ }^{64}$ C. P. Bee,${ }^{84}$ L. J. Beemster, ${ }^{106}$ T. A. Beermann, ${ }^{176}$ M. Begel, ${ }^{25}$ C. Belanger-Champagne, ${ }^{86}$ P. J. Bell, ${ }^{49}$ W. H. Bell, ${ }^{49}$ G. Bella,${ }^{154}$ L. Bellagamba, ${ }^{20 a}$ A. Bellerive,${ }^{29}$ M. Bellomo, ${ }^{30}$

A. Belloni, ${ }^{57}$ O. L. Beloborodova, ${ }^{108, \mathrm{i}} \mathrm{K}$. Belotskiy, ${ }^{97}$ O. Beltramello, ${ }^{30}$ O. Benary, ${ }^{154}$ D. Benchekroun, ${ }^{136 a}$ K. Bendtz, ${ }^{147 a, 147 b}$ N. Benekos, ${ }^{166}$ Y. Benhammou, ${ }^{154}$ E. Benhar Noccioli, ${ }^{49}$ J. A. Benitez Garcia, ${ }^{160 b}$

D. P. Benjamin, ${ }^{45}$ J. R. Bensinger, ${ }^{23}$ K. Benslama, ${ }^{131}$ S. Bentvelsen, ${ }^{106}$ D. Berge,${ }^{30}$ E. Bergeaas Kuutmann, ${ }^{16}$ N. Berger, ${ }^{5}$ F. Berghaus, ${ }^{170}$ E. Berglund, ${ }^{106}$ J. Beringer ${ }^{15}$ C. Bernard ${ }^{22}$ P. Bernat, ${ }^{77}$ R. Bernhard ${ }^{48}$ C. Bernius,${ }^{78}$ F. U. Bernlochner, ${ }^{170}$ T. Berry, ${ }^{76}$ P. Berta, ${ }^{128}$ C. Bertella, ${ }^{84}$ F. Bertolucci, ${ }^{123 a, 123 b}$ M. I. Besana, ${ }^{90 a}$ G. J. Besjes, ${ }^{105}$ O. Bessidskaia, ${ }^{147 a, 147 \mathrm{~b}}$ N. Besson, ${ }^{137}$ S. Bethke, ${ }^{100}$ W. Bhimji, ${ }^{46}$ R. M. Bianchi, ${ }^{124}$ L. Bianchini, ${ }^{23}$ M. Bianco, ${ }^{30}$ O. Biebel, ${ }^{99}$ S. P. Bieniek, ${ }^{77}$ K. Bierwagen,${ }^{54}$ J. Biesiada, ${ }^{15}$ M. Biglietti, ${ }^{135 a}$ J. Bilbao De Mendizabal, ${ }^{49}$ H. Bilokon, ${ }^{47}$ M. Bindi, ${ }^{20 a, 20 b}$ S. Binet, ${ }^{116}$ A. Bingul, ${ }^{19 c}$ C. Bini, ${ }^{133 a, 133 b}$ B. Bittner, ${ }^{100}$ C. W. Black, ${ }^{151}$ J. E. Black,,${ }^{144}$

K. M. Black, ${ }^{22}$ D. Blackburn, ${ }^{139}$ R. E. Blair, ${ }^{6}$ J.-B. Blanchard, ${ }^{137}$ T. Blazek, ${ }^{145 a}$ I. Bloch, ${ }^{42}$ C. Blocker, ${ }^{23}$ J. Blocki, ${ }^{39}$ W. Blum, ${ }^{82, a}$ U. Blumenschein, ${ }^{54}$ G. J. Bobbink, ${ }^{106}$ V. S. Bobrovnikov, ${ }^{108}$ S. S. Bocchetta, ${ }^{80}$ A. Bocci, ${ }^{45}$ C. R. Boddy, ${ }_{119}^{19}$ M. Boehler, ${ }^{48}$ J. Boek, ${ }^{176}$ T. T. Boek, ${ }^{176}$ N. Boelaert,${ }^{36}$ J. A. Bogaerts, ${ }^{30}$ A. G. Bogdanchikov, ${ }^{108}$ A. Bogouch, ${ }^{91, a}$ C. Bohm, ${ }^{147 a}$ J. Bohm, ${ }^{126}$ V. Boisvert, ${ }^{76}$ T. Bold, ${ }^{38 a}$ V. Boldea, ${ }^{26 a}$ A. S. Boldyrev ${ }^{98}$ N. M. Bolnet, ${ }^{137}$ M. Bomben, ${ }^{79}$ M. Bona, ${ }^{75}$ M. Boonekamp,${ }^{137}$ S. Bordoni, ${ }^{79}$ C. Borer,${ }^{17}$ A. Borisov, ${ }^{129}$ G. Borissov, ${ }^{71}$ M. Borri, ${ }^{83}$ S. Borroni, ${ }^{42}$ J. Bortfeldt, ${ }^{99}$ V. Bortolotto, ${ }^{135 a, 135 b}$ K. Bos, ${ }^{106}$ D. Boscherini, ${ }^{20 a}$ M. Bosman, ${ }^{12}$ H. Boterenbrood, ${ }^{106}$ J. Bouchami ${ }^{94}$ J. Boudreau, ${ }^{124}$ E. V. Bouhova-Thacker, ${ }^{71}$ D. Boumediene, ${ }^{34}$ C. Bourdarios, ${ }^{116}$ N. Bousson, ${ }^{84}$

S. Boutouil, ${ }^{136 \mathrm{~d}}$ A. Boveia, ${ }^{31}$ J. Boyd,${ }^{30}$ I. R. Boyko, ${ }^{64}$ I. Bozovic-Jelisavcic, ${ }^{13 \mathrm{~b}}$ J. Bracinik, ${ }^{18}$ P. Branchini ${ }^{135 a}$ A. Brandt,${ }^{8}$ G. Brandt,${ }^{15}$ O. Brandt ${ }^{54}$ U. Bratzler, ${ }^{157}$ B. Brau, ${ }^{85}$ J. E. Brau,${ }^{15}$ H. M. Braun, ${ }^{176, a}$ S. F. Brazzale, ${ }^{165 a, 165 c}$ B. Brelier, ${ }^{159}$ K. Brendlinger, ${ }^{121}$ R. Brenner,${ }^{167}$ S. Bressler, ${ }^{173}$ T. M. Bristow, ${ }^{46}$ D. Britton, ${ }^{53}$ F. M. Brochu, ${ }^{28}$ I. Brock, ${ }^{21}$ R. Brock ${ }^{89}$ F. Broggi, ${ }^{90 a}$ C. Bromberg, ${ }^{89}$ J. Bronner, ${ }^{100}$ G. Brooijmans, ${ }^{35}$ T. Brooks, ${ }^{76}$ W. K. Brooks,${ }^{32 b}$ E. Brost,${ }^{115}$ G. Brown, ${ }^{83}$ J. Brown, ${ }^{55}$ P. A. Bruckman de Renstrom, ${ }^{39}$ D. Bruncko, ${ }^{145 b}$ R. Bruneliere, ${ }^{48}$ S. Brunet, ${ }^{60}$ 
A. Bruni, ${ }^{20 a}$ G. Bruni, ${ }^{20 a}$ M. Bruschi, ${ }^{20 a}$ L. Bryngemark, ${ }^{80}$ T. Buanes, ${ }^{14}$ Q. Buat,${ }^{55}$ F. Bucci, ${ }^{49}$ J. Buchanan, ${ }^{119}$ P. Buchholz ${ }^{142}$ R. M. Buckingham, ${ }^{119}$ A. G. Buckley, ${ }^{46}$ S. I. Buda, ${ }^{26 a}$ I. A. Budagov, ${ }^{64}$ B. Budick,${ }^{109}$ F. Buehrer, ${ }^{48}$ L. Bugge, ${ }^{18}$ O. Bulekov, ${ }^{97}$ A. C. Bundock, ${ }^{73}$ M. Bunse, ${ }^{43}$ H. Burckhart,${ }^{30}$ S. Burdin, ${ }^{73}$ T. Burgess, ${ }^{14}$ S. Burke, ${ }^{130}$ I. Burmeister, ${ }^{43}$ E. Busato, ${ }^{34}$ V. Büscher, ${ }^{82}$ P. Bussey, ${ }^{53}$ C. P. Buszello, ${ }^{167}$ B. Butler, ${ }^{57}$ J. M. Butler, ${ }^{22}$ A. I. Butt, ${ }^{3}$ C. M. Buttar, ${ }^{53}$ J. M. Butterworth, ${ }^{77}$ W. Buttinger, ${ }^{28}$ A. Buzatu, ${ }^{53}$ M. Byszewski, ${ }^{10}$ S. Cabrera Urbán, ${ }^{168}$ D. Caforio, ${ }^{20 a, 20 b}$ O. Cakir, ${ }^{4 a}$ P. Calafiura, ${ }^{15}$ G. Calderini, ${ }^{79}$ P. Calfayan, ${ }^{99}$ R. Calkins, ${ }^{107}$ L. P. Caloba, ${ }^{24 a}$ R. Caloi, ${ }^{133 a, 133 b}$ D. Calvet, ${ }^{34}$ S. Calvet, ${ }^{34}$ R. Camacho Toro, ${ }^{49}$ P. Camarri, ${ }^{134 a, 134 b}$ D. Cameron, ${ }^{118}$ L. M. Caminada, ${ }^{15}$ R. Caminal Armadans, ${ }^{12}$ S. Campana, ${ }^{30}$ M. Campanelli, ${ }^{77}$ V. Canale, ${ }^{103 a, 103 b}$ F. Canelli, ${ }^{31}$ A. Canepa, ${ }^{160 a}$ J. Cantero, ${ }^{81}$ R. Cantrill,${ }^{76}$ T. Cao,${ }^{40}$ M. D. M. Capeans Garrido,${ }^{30}$ I. Caprini, ${ }^{26 a}$ M. Caprini,${ }^{26 a}$ M. Capua, ${ }^{37 a, 37 b}$ R. Caputo, ${ }^{82}$ R. Cardarelli, ${ }^{134 a}$ T. Carli, ${ }^{30}$ G. Carlino, ${ }^{103 a}$ L. Carminati, ${ }^{90 a, 90 b}$ S. Caron, ${ }^{105}$

E. Carquin, ${ }^{32 a}$ G. D. Carrillo-Montoya, ${ }^{146 c}$ A. A. Carter ${ }^{75}$ J. R. Carter,${ }^{28}$ J. Carvalho, ${ }^{125 a, j}$ D. Casadei, ${ }^{77}$ M. P. Casado,${ }^{12}$ C. Caso, ${ }^{50 a, 50 b, a}$ E. Castaneda-Miranda, ${ }^{146 b}$ A. Castelli, ${ }^{106}$ V. Castillo Gimenez, ${ }^{168}$ N. F. Castro, ${ }^{125 a}$ G. Cataldi, ${ }^{72 a}$ P. Catastini, ${ }^{57}$ A. Catinaccio, ${ }^{30}$ J. R. Catmore,${ }^{30}$ A. Cattai,${ }^{30}$ G. Cattani, ${ }^{134 a, 134 b}$ S. Caughron,${ }^{89}$ V. Cavaliere, ${ }^{166}$ D. Cavalli, ${ }^{90 a}$ M. Cavalli-Sforza, ${ }^{12}$ V. Cavasinni, ${ }^{123 a, 123 b}$ F. Ceradini, ${ }^{135 a, 135 b}$ B. Cerio, ${ }^{45}$ A. S. Cerqueira, ${ }^{24 b}$ A. Cerri, ${ }^{15}$ L. Cerrito,${ }^{75}$ F. Cerutti,${ }^{15}$ A. Cervelli, ${ }^{17}$ S. A. Cetin, ${ }^{19 b}$ A. Chafaq, ${ }^{136 a}$ D. Chakraborty, ${ }^{107}$ I. Chalupkova, ${ }^{128}$ K. Chan, ${ }^{3}$ P. Chang, ${ }^{166}$ B. Chapleau, ${ }^{86}$ J. D. Chapman, ${ }^{28}$ J. W. Chapman ${ }^{88}$ D. G. Charlton, ${ }^{18}$ V. Chavda, ${ }^{83}$ C. A. Chavez Barajas, ${ }^{30}$ S. Cheatham, ${ }^{86}$ S. Chekanov, ${ }^{6}$ S. V. Chekulaev, ${ }^{160 a}$ G. A. Chelkov, ${ }^{64}$ M. A. Chelstowska, ${ }^{88}$ C. Chen, ${ }^{63} \mathrm{H}$. Chen, ${ }^{25}$ K. Chen, ${ }^{149}$ S. Chen, ${ }^{33 c}$ X. Chen, ${ }^{174}$ Y. Chen, ${ }^{35}$ Y. Cheng, ${ }^{31}$ A. Cheplakov, ${ }^{64}$ R. Cherkaoui El Moursli, ${ }^{136 e}$ V. Chernyatin, ${ }^{25, a}$ E. Cheu, ${ }^{7}$ L. Chevalier, ${ }^{137}$ V. Chiarella, ${ }^{47}$ G. Chiefari, ${ }^{103 a, 103 b}$ J. T. Childers, ${ }^{30}$ A. Chilingarov, ${ }^{71}$ G. Chiodini, ${ }^{72 a}$ A. S. Chisholm, ${ }^{18}$ R. T. Chislett, ${ }^{77}$ A. Chitan, ${ }^{26 a}$ M. V. Chizhov, ${ }^{64}$ G. Choudalakis, ${ }^{31}$ S. Chouridou, ${ }^{9}$ B. K. B. Chow, ${ }^{99}$ I. A. Christidi, ${ }^{77}$ A. Christov, ${ }^{48}$ D. Chromek-Burckhart,${ }^{30}$ M. L. Chu ${ }^{152}$ J. Chudoba,${ }^{126}$ G. Ciapetti,${ }^{133 a, 133 b}$ A. K. Ciftci, ${ }^{4 a}$ R. Ciftci,${ }^{4 a}$ D. Cinca, ${ }^{62}$ V. Cindro, ${ }^{74}$ A. Ciocio, ${ }^{15}$ M. Cirilli, ${ }^{88}$ P. Cirkovic, ${ }^{13 b}$ Z. H. Citron, ${ }^{173}$ M. Citterio, ${ }^{90 a}$ M. Ciubancan, ${ }^{26 a}$ A. Clark, ${ }^{49}$ P. J. Clark, ${ }^{46}$ R. N. Clarke, ${ }^{15}$ J. C. Clemens, ${ }^{84}$ B. Clement, ${ }^{55}$ C. Clement, ${ }^{147 a, 147 b}$ Y. Coadou, ${ }^{84}$ M. Cobal, ${ }^{165 a, 165 \mathrm{c}}$ A. Coccaro, ${ }^{139} \mathrm{~J}$. Cochran, ${ }^{63}$ S. Coelli, ${ }^{90 \mathrm{a}}$ L. Coffey,${ }^{23} \mathrm{~J}$. G. Cogan, ${ }^{144} \mathrm{~J}$. Coggeshall, ${ }^{166} \mathrm{~J}$. Colas, ${ }^{5}$ B. Cole,${ }^{35}$ S. Cole,${ }^{107}$ A. P. Colijn, ${ }^{106}$ C. Collins-Tooth, ${ }^{53}$ J. Collot, ${ }^{55}$ T. Colombo,${ }^{58 c}$ G. Colon, ${ }^{85}$ G. Compostella,${ }^{100}$ P. Conde Muiño, ${ }^{125 a}$ E. Coniavitis, ${ }^{167}$ M. C. Conidi, ${ }^{12}$ S. M. Consonni, ${ }^{90 a, 90 b}$ V. Consorti, ${ }^{48}$ S. Constantinescu, ${ }^{26 a}$ C. Conta, ${ }^{120 a, 120 b}$ G. Conti, ${ }^{57}$ F. Conventi, ${ }^{103 a, k}$ M. Cooke, ${ }^{15}$ B. D. Cooper, ${ }^{77}$ A. M. Cooper-Sarkar, ${ }^{119}$

N. J. Cooper-Smith, ${ }^{76}$ K. Copic, ${ }^{15}$ T. Cornelissen, ${ }^{176}$ M. Corradi, ${ }^{20 a}$ F. Corriveau, ${ }^{86,1}$ A. Corso-Radu, ${ }^{164}$ A. Cortes-Gonzalez, ${ }^{12}$ G. Cortiana, ${ }^{100}$ G. Costa, ${ }^{90 a}$ M. J. Costa, ${ }^{168}$ D. Costanzo,${ }^{140}$ D. Côté, ${ }^{8}$ G. Cottin, ${ }^{32 a}$ L. Courneyea, ${ }^{170}$ G. Cowan,${ }^{76}$ B. E. Cox,${ }^{83}$ K. Cranmer, ${ }^{109}$ G. Cree, ${ }^{29}$ S. Crépé-Renaudin, ${ }^{55}$ F. Crescioli, ${ }^{79}$ M. Cristinziani, ${ }^{21}$ G. Crosetti, ${ }^{37 a, 37 b}$ C.-M. Cuciuc, ${ }^{26 a}$ C. Cuenca Almenar, ${ }^{177}$ T. Cuhadar Donszelmann, ${ }^{140}$ J. Cummings, ${ }^{177}$ M. Curatolo, ${ }^{47}$ C. Cuthbert, ${ }^{151}$ H. Czirr,,${ }^{142}$ P. Czodrowski, ${ }^{44}$ Z. Czyczula, ${ }^{177}$ S. D' Auria, ${ }^{53}$ M. D’Onofrio, ${ }^{73}$ A. D’Orazio, ${ }^{133 a, 133 b}$ M. J. Da Cunha Sargedas De Sousa, ${ }^{125 a}$ C. Da Via, ${ }^{83}$ W. Dabrowski, ${ }^{38 a}$ A. Dafinca, ${ }^{119}$ T. Dai, ${ }^{88}$ F. Dallaire,${ }^{94}$ C. Dallapiccola,${ }^{85}$ M. Dam, ${ }^{36}$ D. S. Damiani, ${ }^{138}$ A. C. Daniells,${ }^{18}$ V. Dao, ${ }^{105}$ G. Darbo, ${ }^{50 a}$ G. L. Darlea, ${ }^{26 c}$ S. Darmora, ${ }^{8}$ J. A. Dassoulas,${ }^{42}$ W. Davey, ${ }^{21}$ C. David, ${ }^{170}$ T. Davidek, ${ }^{128}$ E. Davies,${ }^{19, f}$ M. Davies,${ }^{94}$ O. Davignon, ${ }^{79}$ A. R. Davison, ${ }^{77}$ Y. Davygora, ${ }^{58 a}$ E. Dawe, ${ }^{143}$ I. Dawson,,${ }^{140}$

R. K. Daya-Ishmukhametova, ${ }^{23}$ K. De,${ }^{8}$ R. de Asmundis, ${ }^{103 a}$ S. De Castro, ${ }^{20 a, 20 b}$ S. De Cecco, ${ }^{79}$ J. de Graat, ${ }^{99}$ N. De Groot, ${ }^{105}$ P. de Jong, ${ }^{106}$ C. De La Taille, ${ }^{116}$ H. De la Torre, ${ }^{81}$ F. De Lorenzi,${ }^{63}$ L. De Nooij, ${ }^{106}$ D. De Pedis, ${ }^{133 a}$

A. De Salvo, ${ }^{133 a}$ U. De Sanctis, ${ }^{165 a, 165 c}$ A. De Santo, ${ }^{150}$ J. B. De Vivie De Regie, ${ }^{116}$ G. De Zorzi,

W. J. Dearnaley, ${ }^{71}$ R. Debbe, ${ }^{25}$ C. Debenedetti, ${ }^{46}$ B. Dechenaux,${ }^{55}$ D. V. Dedovich,${ }^{64}$ J. Degenhardt, ${ }^{121}$ J. Del Peso, ${ }^{81}$

T. Del Prete, ${ }^{123 a, 123 b}$ T. Delemontex,${ }^{55}$ F. Deliot, ${ }^{137}$ M. Deliyergiyev, ${ }^{74}$ A. Dell'Acqua,${ }^{30}$ L. Dell'Asta, ${ }^{22}$ M. Della Pietra, ${ }^{103 a, k}$ D. della Volpe,${ }^{103 a, 103 b}$ M. Delmastro, ${ }^{5}$ P. A. Delsart ${ }^{55}$ C. Deluca, ${ }^{106}$ S. Demers,${ }^{177}$ M. Demichev ${ }^{64}$ A. Demilly, ${ }^{79}$ B. Demirkoz, ${ }^{12, m}$ S. P. Denisov,${ }^{129}$ D. Derendarz, ${ }^{39}$ J. E. Derkaoui, ${ }^{136 d}$ F. Derue,${ }^{79}$ P. Dervan, ${ }^{73}$ K. Desch, ${ }^{21}$ P. O. Deviveiros, ${ }^{106}$ A. Dewhurst ${ }^{130}$ B. DeWilde, ${ }^{149}$ S. Dhaliwal, ${ }^{106}$ R. Dhullipudi, ${ }^{78, n}$ A. Di Ciaccio, ${ }^{134 a, 134 b}$ L. Di Ciaccio,${ }^{5}$ C. Di Donato, ${ }^{103 a, 103 b}$ A. Di Girolamo, ${ }^{30}$ B. Di Girolamo, ${ }^{30}$ A. Di Mattia,${ }^{153}$

B. Di Micco, ${ }^{135 a, 135 b}$ R. Di Nardo, ${ }^{47}$ A. Di Simone,${ }^{48}$ R. Di Sipio, ${ }^{20 a, 20 b}$ D. Di Valentino, ${ }^{29}$ M. A. Diaz ${ }^{32 a}$

E. B. Diehl ${ }^{88}$ J. Dietrich, ${ }^{42}$ T. A. Dietzsch ${ }^{58 a}$ S. Diglio, ${ }^{87}$ K. Dindar Yagci,${ }^{40}$ J. Dingfelder, ${ }^{21}$ C. Dionisi, ${ }^{133 a, 133 b}$ P. Dita, ${ }^{26 a}$ S. Dita, ${ }^{26 a}$ F. Dittus,${ }^{30}$ F. Djama ${ }^{84}$ T. Djobava,${ }^{51 b}$ M. A. B. do Vale,${ }^{24 c}$ A. Do Valle Wemans, ${ }^{125 a, o}$ T. K. O. Doan, ${ }^{5}$ D. Dobos,${ }^{30}$ E. Dobson, ${ }^{77}$ J. Dodd,${ }^{35}$ C. Doglioni,${ }^{49}$ T. Doherty, ${ }^{53}$ T. Dohmae, ${ }^{156}$ Y. Doi, ${ }^{65, a}$ J. Dolejsi, ${ }^{128}$ Z. Dolezal, ${ }^{128}$ B. A. Dolgoshein,,${ }^{97}$ M. Donadelli, ${ }^{24 \mathrm{~d}}$ S. Donati, ${ }^{123 a, 123 b}$ J. Donini, ${ }^{34}$ J. Dopke, ${ }^{30}$ 
A. Doria, ${ }^{103 a}$ A. Dos Anjos, ${ }^{174}$ A. Dotti, ${ }^{123 a, 123 b}$ M. T. Dova, ${ }^{70}$ A. T. Doyle,${ }^{53}$ M. Dris,${ }^{10}$ J. Dubbert,${ }^{88}$ S. Dube, ${ }^{15}$ E. Dubreuil,${ }^{34}$ E. Duchovni, ${ }^{173}$ G. Duckeck,${ }^{99}$ D. Duda ${ }^{176}$ A. Dudarev, ${ }^{30}$ F. Dudziak, ${ }^{63}$ L. Duflot, ${ }^{116}$ L. Duguid, ${ }^{76}$ M. Dührssen, ${ }^{30}$ M. Dunford ${ }^{58 a}$ H. Duran Yildiz, ${ }^{4 a}$ M. Düren,${ }^{52}$ M. Dwuznik,${ }^{38 a}$ J. Ebke, ${ }^{99}$ W. Edson, ${ }^{2}$ C. A. Edwards ${ }^{76}$ N. C. Edwards,${ }^{46}$ W. Ehrenfeld, ${ }^{21}$ T. Eifert, ${ }^{144}$ G. Eigen, ${ }^{14}$ K. Einsweiler,${ }^{15}$ E. Eisenhandler ${ }^{75}$ T. Ekelof, ${ }^{167}$ M. El Kacimi, ${ }^{136 c}$ M. Ellert, ${ }^{167}$ S. Elles, ${ }^{5}$ F. Ellinghaus, ${ }^{82}$ K. Ellis,${ }^{75}$ N. Ellis, ${ }^{30}$ J. Elmsheuser, ${ }^{99}$ M. Elsing, ${ }^{30}$ D. Emeliyanov, ${ }^{130}$ Y. Enari, ${ }^{156}$ O.C. Endner, ${ }^{82}$ R. Engelmann, ${ }^{149}$ A. Engl, ${ }^{99}$ J. Erdmann, ${ }^{177}$ A. Ereditato, ${ }^{17}$ D. Eriksson, ${ }^{147 a}$ G. Ernis,${ }^{176}$ J. Ernst, ${ }^{2}$ M. Ernst, ${ }^{25}$ J. Ernwein, ${ }^{137}$ D. Errede, ${ }^{166}$ S. Errede, ${ }^{166}$ E. Ertel,${ }^{82}$ M. Escalier,,${ }^{116}$ H. Esch,${ }^{43}$ C. Escobar, ${ }^{124}$ X. Espinal Curull, ${ }^{12}$ B. Esposito, ${ }^{47}$ F. Etienne, ${ }^{84}$ A. I. Etienvre,${ }^{137}$ E. Etzion, ${ }^{154}$ D. Evangelakou, ${ }^{54}$ H. Evans,${ }^{60}$ L. Fabbri, ${ }^{20 a, 20 b}$ G. Facini,${ }^{30}$ R. M. Fakhrutdinov,${ }^{129}$ S. Falciano, ${ }^{133 a}$

Y. Fang, ${ }^{33 a}$ M. Fanti, ${ }^{90 a, 90 b}$ A. Farbin, ${ }^{8}$ A. Farilla, ${ }^{135 a}$ T. Farooque, ${ }^{159}$ S. Farrell, ${ }^{164}$ S. M. Farrington, ${ }^{171}$

P. Farthouat,${ }^{30}$ F. Fassi, ${ }^{168}$ P. Fassnacht,${ }^{30}$ D. Fassouliotis, ${ }^{9}$ B. Fatholahzadeh, ${ }^{159}$ A. Favareto, ${ }^{50 a, 50 b}$ L. Fayard, ${ }^{116}$ P. Federic, ${ }^{145 a}$ O. L. Fedin, ${ }^{122}$ W. Fedorko, ${ }^{169}$ M. Fehling-Kaschek,${ }^{48}$ L. Feligioni,${ }^{84}$ C. Feng, ${ }^{33 d}$ E. J. Feng, ${ }^{6}$ H. Feng, ${ }^{88}$ A. B. Fenyuk, ${ }^{129}$ J. Ferencei, ${ }^{145 b}$ W. Fernando, ${ }^{6}$ S. Ferrag, ${ }^{53}$ J. Ferrando, ${ }^{53}$ V. Ferrara, ${ }^{42}$ A. Ferrari, ${ }^{167}$ P. Ferrari, ${ }^{106}$ R. Ferrari, ${ }^{120 a}$ D. E. Ferreira de Lima ${ }^{53}$ A. Ferrer, ${ }^{168}$ D. Ferrere, ${ }^{49}$ C. Ferretti, ${ }^{88}$

A. Ferretto Parodi, ${ }^{50 a, 50 b}$ M. Fiascaris,${ }^{31}$ F. Fiedler, ${ }^{82}$ A. Filipčič,${ }^{74}$ M. Filipuzzi, ${ }^{42}$ F. Filthaut, ${ }^{105}$ M. Fincke-Keeler, ${ }^{170}$ K. D. Finelli, ${ }^{45}$ M. C. N. Fiolhais, ${ }^{125 a, j}$ L. Fiorini, ${ }^{168}$ A. Firan, ${ }^{40}$ J. Fischer, ${ }^{176}$ M. J. Fisher, ${ }^{110}$ E. A. Fitzgerald, ${ }^{23}$ M. Flechl, ${ }^{48}$ I. Fleck, ${ }^{142}$ P. Fleischmann, ${ }^{175}$ S. Fleischmann, ${ }^{176}$ G. T. Fletcher, ${ }^{140}$ G. Fletcher, ${ }^{75}$ T. Flick, ${ }^{176}$ A. Floderus, ${ }^{80}$ L. R. Flores Castillo, ${ }^{174}$ A. C. Florez Bustos, ${ }^{160 b}$ M. J. Flowerdew, ${ }^{100}$ T. Fonseca Martin, ${ }^{17}$ A. Formica, ${ }^{137}$ A. Forti, ${ }^{83}$ D. Fortin, ${ }^{160 a}$ D. Fournier, ${ }^{116}$ H. Fox,${ }^{71}$ P. Francavilla, ${ }^{12}$ M. Franchini, ${ }^{20 a, 20 b}$

S. Franchino, ${ }^{30}$ D. Francis,${ }^{30}$ M. Franklin,${ }^{57}$ S. Franz, ${ }^{61}$ M. Fraternali, ${ }^{120 a, 120 b}$ S. Fratina, ${ }^{121}$ S. T. French,${ }^{28}$ C. Friedrich ${ }^{42}$ F. Friedrich, ${ }^{44}$ D. Froidevaux,${ }^{30}$ J. A. Frost,${ }^{28}$ C. Fukunaga,${ }^{157}$ E. Fullana Torregrosa, ${ }^{128}$ B. G. Fulsom, ${ }^{144}$ J. Fuster, ${ }^{168}$ C. Gabaldon, ${ }^{55}$ O. Gabizon, ${ }^{173}$ A. Gabrielli, ${ }^{20 a, 20 b}$ A. Gabrielli, ${ }^{133 a, 133 b}$ S. Gadatsch, ${ }^{106}$ T. Gadfort, ${ }^{25}$ S. Gadomski, ${ }^{49}$ G. Gagliardi, ${ }^{50 a, 50 b}$ P. Gagnon, ${ }^{60}$ C. Galea,${ }^{99}$ B. Galhardo, ${ }^{125 a}$ E. J. Gallas, ${ }^{119}$ V. Gallo, ${ }^{17}$ B. J. Gallop, ${ }^{130}$ P. Gallus, ${ }^{127}$ G. Galster, ${ }^{36}$ K. K. Gan, ${ }^{110}$ R. P. Gandrajula, ${ }^{62}$ J. Gao, ${ }^{33 b, p}$ Y. S. Gao, ${ }^{144, h}$ F. M. Garay Walls, ${ }^{46}$ F. Garberson, ${ }^{177}$ C. García, ${ }^{168}$ J. E. García Navarro, ${ }^{168}$ M. Garcia-Sciveres,${ }^{15}$ R. W. Gardner,${ }^{31}$ N. Garelli, ${ }^{144}$ V. Garonne, ${ }^{30}$ C. Gatti, ${ }^{47}$ G. Gaudio, ${ }^{120 a}$ B. Gaur, ${ }^{142}$ L. Gauthier, ${ }^{94}$ P. Gauzzi, ${ }^{133 a, 133 b}$ I. L. Gavrilenko, ${ }^{95}$ C. Gay, ${ }^{169}$ G. Gaycken,${ }^{21}$ E. N. Gazis,${ }^{10}$ P. Ge,${ }^{33 d, q}$ Z. Gecse,${ }^{169}$ C. N. P. Gee, ${ }^{130}$ D. A. A. Geerts, ${ }^{106}$ Ch. Geich-Gimbel,${ }^{21}$ K. Gellerstedt, ${ }^{147 a, 147 b}$ C. Gemme,${ }^{50 a}$ A. Gemmell,${ }^{53}$ M. H. Genest, ${ }^{55}$ S. Gentile, ${ }^{133 a, 133 b}$ M. George ${ }^{54}$ S. George,${ }^{76}$ D. Gerbaudo, ${ }^{164}$ A. Gershon, ${ }^{154}$ H. Ghazlane, ${ }^{136 b}$ N. Ghodbane, ${ }^{34}$ B. Giacobbe, ${ }^{20 a}$ S. Giagu, ${ }^{133 a, 133 b}$ V. Giangiobbe,${ }^{12}$ P. Giannetti, ${ }^{123 a, 123 b}$ F. Gianotti, ${ }^{30}$ B. Gibbard ${ }^{25}$ S. M. Gibson, ${ }^{76}$ M. Gilchriese, ${ }^{15}$ T. P. S. Gillam, ${ }^{28}$ D. Gillberg, ${ }^{30}$ A. R. Gillman, ${ }^{130}$ D. M. Gingrich, ${ }^{3, g}$ N. Giokaris, ${ }^{9}$

M. P. Giordani, ${ }^{165 \mathrm{c}}$ R. Giordano, ${ }^{103 a, 103 \mathrm{~b}}$ F. M. Giorgi, ${ }^{16}$ P. Giovannini, ${ }^{100}$ P. F. Giraud, ${ }^{137}$ D. Giugni, ${ }^{90 a}$ C. Giuliani, ${ }^{48}$ M. Giunta, ${ }^{94}$ B. K. Gjelsten, ${ }^{118}$ I. Gkialas, ${ }^{155, \mathrm{r}}$ L. K. Gladilin, ${ }^{98}$ C. Glasman, ${ }^{81}$ J. Glatzer, ${ }^{21}$ A. Glazov, ${ }^{42}$ G. L. Glonti, ${ }^{64}$ M. Goblirsch-Kolb, ${ }^{100}$ J. R. Goddard ${ }^{75}$ J. Godfrey, ${ }^{143}$ J. Godlewski, ${ }^{30}$ C. Goeringer, ${ }^{82}$ S. Goldfarb, ${ }^{88}$ T. Golling, ${ }^{177}$ D. Golubkov, ${ }^{129}$ A. Gomes, ${ }^{125 a, e}$ L. S. Gomez Fajardo, ${ }^{42}$ R. Gonçalo, ${ }^{76}$

J. Goncalves Pinto Firmino Da Costa, ${ }^{42}$ L. Gonella, ${ }^{21}$ S. González de la Hoz,${ }^{168}$ G. Gonzalez Parra, ${ }^{12}$

M. L. Gonzalez Silva, ${ }^{27}$ S. Gonzalez-Sevilla, ${ }^{49}$ J. J. Goodson, ${ }^{149}$ L. Goossens,${ }^{30}$ P. A. Gorbounov, ${ }^{96}$ H. A. Gordon, ${ }^{25}$ I. Gorelov, ${ }^{104}$ G. Gorfine, ${ }^{176}$ B. Gorini, ${ }^{30}$ E. Gorini, ${ }^{72 a, 72 b}$ A. Gorišek, ${ }^{74}$ E. Gornicki, ${ }^{39}$ A. T. Goshaw, ${ }^{6}$ C. Gössling,${ }^{43}$ M. I. Gostkin, ${ }^{64}$ I. Gough Eschrich, ${ }^{164}$ M. Gouighri, ${ }^{136 a}$ D. Goujdami, ${ }^{136 c}$ M. P. Goulette, ${ }^{49}$ A. G. Goussiou, ${ }^{139}$ C. Goy, ${ }^{5}$ S. Gozpinar, ${ }^{23}$ H. M. X. Grabas, ${ }^{137}$ L. Graber, ${ }^{54}$ I. Grabowska-Bold, ${ }^{38 a}$ P. Grafström, ${ }^{20 a, 20 b}$ K-J. Grahn, ${ }^{42}$ J. Gramling, ${ }^{49}$ E. Gramstad, ${ }^{118}$ F. Grancagnolo, ${ }^{72 a}$ S. Grancagnolo, ${ }^{16}$ V. Grassi, ${ }^{149}$ V. Gratchev, ${ }^{122}$ H. M. Gray, ${ }^{30}$ J. A. Gray, ${ }^{149}$ E. Graziani, ${ }^{135 a}$ O. G. Grebenyuk, ${ }^{122}$ Z. D. Greenwood, ${ }^{78, n}$ K. Gregersen, ${ }^{36}$ I. M. Gregor, ${ }^{42}$ P. Grenier, ${ }^{144}$ J. Griffiths, ${ }^{8}$ N. Grigalashvili, ${ }^{64}$ A. A. Grillo, ${ }^{138}$ K. Grimm, ${ }^{71}$ S. Grinstein, ${ }^{12, s} \mathrm{Ph}$. Gris,${ }^{34}$ Y. V. Grishkevich, ${ }^{98}$ J.-F. Grivaz, ${ }^{116}$ J. P. Grohs, ${ }^{44}$ A. Grohsjean, ${ }^{42}$ E. Gross, ${ }^{173}$ J. Grosse-Knetter, ${ }^{54}$ J. Groth-Jensen, ${ }^{173}$ Z. J. Grout, ${ }^{150}$ K. Grybel, ${ }^{142}$ F. Guescini, ${ }^{49}$ D. Guest,${ }^{177}$ O. Gueta, ${ }^{154}$ C. Guicheney, ${ }^{34}$ E. Guido, ${ }^{50 a, 50 b}$ T. Guillemin, ${ }^{116}$ S. Guindon, ${ }^{2}$ U. Gul, ${ }^{53}$ C. Gumpert, ${ }^{44}$ J. Gunther, ${ }^{127}$ J. Guo, ${ }^{35}$ S. Gupta, ${ }^{119}$ P. Gutierrez ${ }^{112}$ N. G. Gutierrez Ortiz,${ }^{53}$ C. Gutschow, ${ }^{77}$ N. Guttman, ${ }^{154}$ O. Gutzwiller, ${ }^{174}$ C. Guyot, ${ }^{137}$ C. Gwenlan, ${ }^{119}$ C. B. Gwilliam, ${ }^{73}$ A. Haas, ${ }^{109}$ C. Haber, ${ }^{15}$ H. K. Hadavand,${ }^{8}$ P. Haefner, ${ }^{21}$ S. Hageboeck,${ }^{21}$ Z. Hajduk, ${ }^{39}$ H. Hakobyan, ${ }^{178}$ D. Hall, ${ }^{119}$ G. Halladjian, ${ }^{62}$ K. Hamacher, ${ }^{176}$ P. Hamal, ${ }^{114}$ K. Hamano, ${ }^{87}$ M. Hamer,${ }^{54}$ A. Hamilton, ${ }^{146 a, t}$ S. Hamilton, ${ }^{162}$ L. Han,${ }^{33 b}$ K. Hanagaki, ${ }^{117}$ K. Hanawa,${ }^{156}$ M. Hance, ${ }^{15}$ C. Handel, ${ }^{82}$ P. Hanke, ${ }^{58 a}$ J. R. Hansen, ${ }^{36}$ J. B. Hansen, ${ }^{36}$ J. D. Hansen, ${ }^{36}$ P. H. Hansen, ${ }^{36}$ P. Hansson, ${ }^{144}$ K. Hara ${ }^{161}$ A. S. Hard, ${ }^{174}$ 
T. Harenberg, ${ }^{176}$ S. Harkusha, ${ }^{91}$ D. Harper, ${ }^{88}$ R. D. Harrington, ${ }^{46}$ O. M. Harris, ${ }^{139}$ P. F. Harrison, ${ }^{171}$ F. Hartjes, ${ }^{106}$ A. Harvey,${ }^{56}$ S. Hasegawa, ${ }^{102}$ Y. Hasegawa,${ }^{141}$ S. Hassani, ${ }^{137}$ S. Haug, ${ }^{17}$ M. Hauschild, ${ }^{30}$ R. Hauser, ${ }^{89}$ M. Havranek, ${ }^{21}$ C. M. Hawkes, ${ }^{18}$ R. J. Hawkings, ${ }^{30}$ A. D. Hawkins, ${ }^{80}$ T. Hayashi, ${ }^{161}$ D. Hayden,${ }^{89}$ C. P. Hays, ${ }^{119}$ H. S. Hayward ${ }^{73}$ S. J. Haywood, ${ }^{130}$ S. J. Head ${ }^{18}$ T. Heck, ${ }^{82}$ V. Hedberg,${ }^{80}$ L. Heelan, ${ }^{8}$ S. Heim, ${ }^{121}$ B. Heimel, ${ }^{142}$ B. Heinemann, ${ }^{15}$ S. Heisterkamp,${ }^{36}$ J. Hejbal, ${ }^{126}$ L. Helary, ${ }^{22}$ C. Heller, ${ }^{99}$ M. Heller, ${ }^{30}$ R. E. Heller, ${ }^{15}$ S. Hellman, ${ }^{147 a, 147 b}$ D. Hellmich, ${ }^{21}$ C. Helsens, ${ }^{30}$ J. Henderson, ${ }^{119}$ R. C. W. Henderson, ${ }^{71}$ A. Henrichs, ${ }^{177}$ A. M. Henriques Correia, ${ }^{30}$ S. Henrot-Versille, ${ }^{116}$ C. Hensel,${ }^{54}$ G. H. Herbert, ${ }^{16}$ C. M. Hernandez, ${ }^{8}$

Y. Hernández Jiménez, ${ }^{168}$ R. Herrberg-Schubert, ${ }^{16}$ G. Herten, ${ }^{48}$ R. Hertenberger, ${ }^{99}$ L. Hervas, ${ }^{30}$ G. G. Hesketh ${ }^{77}$ N. P. Hessey, ${ }^{106}$ R. Hickling, ${ }^{75}$ E. Higón-Rodriguez, ${ }^{168}$ J. C. Hill, ${ }^{28}$ K. H. Hiller, ${ }^{42}$ S. Hillert, ${ }^{21}$ S. J. Hillier, ${ }^{18}$ I. Hinchliffe, ${ }^{15}$ E. Hines,${ }^{121}$ M. Hirose,${ }^{117}$ D. Hirschbuehl, ${ }^{176}$ J. Hobbs, ${ }^{149}$ N. Hod, ${ }^{106}$ M. C. Hodgkinson, ${ }^{140}$ P. Hodgson, ${ }^{140}$ A. Hoecker, ${ }^{30}$ M. R. Hoeferkamp, ${ }^{104}$ J. Hoffman, ${ }^{40}$ D. Hoffmann, ${ }^{84}$ J. I. Hofmann, ${ }^{58 a}$ M. Hohlfeld, ${ }^{82}$ S. O. Holmgren, ${ }^{147 a}$ T. M. Hong, ${ }^{121}$ L. Hooft van Huysduynen, ${ }^{109}$ J-Y. Hostachy,${ }^{55}$ S. Hou, ${ }^{152}$ A. Hoummada, ${ }^{136 a}$ J. Howard, ${ }^{119}$ J. Howarth, ${ }^{83}$ M. Hrabovsky, ${ }^{114}$ I. Hristova, ${ }^{16}$ J. Hrivnac, ${ }^{116}$ T. Hryn'ova, ${ }^{5}$ P. J. Hsu, ${ }^{82}$ S.-C. Hsu, ${ }^{139}$ D. Hu, ${ }^{35}$ X. Hu,${ }^{25}$ Y. Huang, ${ }^{146 c}$ Z. Hubacek, ${ }^{30}$ F. Hubaut, ${ }^{84}$ F. Huegging, ${ }^{21}$ A. Huettmann, ${ }^{42}$ T. B. Huffman, ${ }^{119}$ E. W. Hughes, ${ }^{35}$ G. Hughes, ${ }^{71}$ M. Huhtinen, ${ }^{30}$ T. A. Hülsing, ${ }^{82}$ M. Hurwitz, ${ }^{15}$ N. Huseynov, ${ }^{64, d}$ J. Huston, ${ }^{89}$ J. Huth, ${ }^{57}$ G. Iacobucci, ${ }^{49} \mathrm{G}$. Iakovidis, ${ }^{10}$ I. Ibragimov, ${ }^{142} \mathrm{~L}$. Iconomidou-Fayard, ${ }^{116} \mathrm{~J}$. Idarraga, ${ }^{116} \mathrm{P}$. Iengo, ${ }^{103 a}$ O. Igonkina, ${ }^{106}$ T. Iizawa, ${ }^{172}$ Y. Ikegami, ${ }^{65} \mathrm{~K}$. Ikematsu, ${ }^{142} \mathrm{M}$. Ikeno, ${ }^{65} \mathrm{D}$. Iliadis, ${ }^{155} \mathrm{~N}$. Ilic, ${ }^{159}$ Y. Inamaru, ${ }^{66} \mathrm{~T}$. Ince, ${ }^{100}$ P. Ioannou, ${ }^{9}$ M. Iodice, ${ }^{135 a} \mathrm{~K}$. Iordanidou, ${ }^{9}$ V. Ippolito, ${ }^{133 a, 133 b}$ A. Irles Quiles, ${ }^{168} \mathrm{C}$. Isaksson, ${ }^{167} \mathrm{M}$. Ishino,${ }^{67} \mathrm{M}$. Ishitsuka, ${ }^{158}$ R. Ishmukhametov, ${ }^{110} \mathrm{C}$. Issever ${ }^{119} \mathrm{~S}$. Istin, ${ }^{19 a} \mathrm{~A}$. V. Ivashin, ${ }^{129} \mathrm{~W}$. Iwanski, ${ }^{39} \mathrm{H}$. Iwasaki, ${ }^{65} \mathrm{~J}$. M. Izen,${ }^{41} \mathrm{~V}$. Izzo, ${ }^{103 a}$ B. Jackson, ${ }^{121}$ J. N. Jackson, ${ }^{73}$ M. Jackson, ${ }^{73}$ P. Jackson, ${ }^{1}$ M. R. Jaekel, ${ }^{30}$ V. Jain, ${ }^{2}$ K. Jakobs, ${ }^{48}$ S. Jakobsen, ${ }^{36}$ T. Jakoubek ${ }^{126}$ J. Jakubek,${ }^{127}$ D. O. Jamin, ${ }^{152}$ D. K. Jana, ${ }^{112}$ E. Jansen,${ }^{77}$ H. Jansen, ${ }^{30}$ J. Janssen, ${ }^{21}$ M. Janus, ${ }^{171}$ R. C. Jared,${ }^{174}$ G. Jarlskog, ${ }^{80}$ L. Jeanty,${ }^{57}$ G.-Y. Jeng, ${ }^{151}$ I. Jen-La Plante, ${ }^{31}$ D. Jennens, ${ }^{87}$ P. Jenni, ${ }^{48, u}$ J. Jentzsch,${ }^{43}$ C. Jeske, ${ }^{171} \mathrm{~S}$. Jézéquel, ${ }^{5} \mathrm{M}$. K. Jha, ${ }^{20 \mathrm{a}} \mathrm{H}$. Ji, ${ }^{174} \mathrm{~W}$. Ji, ${ }^{82} \mathrm{~J}$. Jia, ${ }^{149}$ Y. Jiang, ${ }^{33 \mathrm{~b}} \mathrm{M}$. Jimenez Belenguer, ${ }^{42} \mathrm{~S}$. Jin, ${ }^{33 \mathrm{a}}$ O. Jinnouchi, ${ }^{158}$ M. D. Joergensen, ${ }^{36}$ D. Joffe, ${ }^{40}$ K. E. Johansson, ${ }^{147 a}$ P. Johansson, ${ }^{140}$ K. A. Johns, ${ }^{7}$

K. Jon-And, ${ }^{147 a, 147 b}$ G. Jones, ${ }^{171}$ R. W. L. Jones, ${ }^{71}$ T. J. Jones, ${ }^{73}$ P. M. Jorge, ${ }^{125 a}$ K. D. Joshi, ${ }^{83}$ J. Jovicevic, ${ }^{148}$ X. Ju, ${ }^{174}$ C. A. Jung, ${ }^{43}$ R. M. Jungst, ${ }^{30}$ P. Jussel,${ }^{61}$ A. Juste Rozas, ${ }^{12, s}$ M. Kaci, ${ }^{168}$ A. Kaczmarska, ${ }^{39}$ P. Kadlecik, ${ }^{36}$ M. Kado, ${ }^{116}$ H. Kagan, ${ }^{110}$ M. Kagan, ${ }^{144}$ E. Kajomovitz,${ }^{45}$ S. Kalinin, ${ }^{176}$ S. Kama, ${ }^{40}$ N. Kanaya, ${ }^{156}$ M. Kaneda ${ }^{30}$ S. Kaneti, ${ }^{28}$ T. Kanno, ${ }^{158}$ V. A. Kantserov, ${ }^{97}$ J. Kanzaki, ${ }^{65}$ B. Kaplan, ${ }^{109}$ A. Kapliy, ${ }^{31}$ D. Kar, ${ }^{53}$ K. Karakostas, ${ }^{10}$ N. Karastathis, ${ }^{10}$ M. Karnevskiy, ${ }^{82}$ S. N. Karpov, ${ }^{64}$ K. Karthik, ${ }^{109}$ V. Kartvelishvili, ${ }^{71}$ A. N. Karyukhin, ${ }^{129}$

L. Kashif, ${ }^{174}$ G. Kasieczka, ${ }^{58 b}$ R. D. Kass, ${ }^{110}$ A. Kastanas, ${ }^{14}$ Y. Kataoka, ${ }^{156}$ A. Katre, ${ }^{49}$ J. Katzy, ${ }^{42}$ V. Kaushik, ${ }^{7}$ K. Kawagoe, ${ }^{69}$ T. Kawamoto, ${ }^{156}$ G. Kawamura ${ }^{54}$ S. Kazama, ${ }^{156}$ V. F. Kazanin, ${ }^{108}$ M. Y. Kazarinov, ${ }^{64}$ R. Keeler, ${ }^{170}$ P. T. Keener, ${ }^{121}$ R. Kehoe, ${ }^{40}$ M. Keil,${ }^{54}$ J. S. Keller, ${ }^{139}$ H. Keoshkerian, ${ }^{5}$ O. Kepka, ${ }^{126}$ B. P. Kerševan, ${ }^{74}$ S. Kersten, ${ }^{176}$ K. Kessoku, ${ }^{156}$ J. Keung, ${ }^{159}$ F. Khalil-zada, ${ }^{11}$ H. Khandanyan, ${ }^{147,147 b}$ A. Khanov, ${ }^{113}$ D. Kharchenko, ${ }^{64}$ A. Khodinov, ${ }^{97}$ A. Khomich, ${ }^{58 \mathrm{a}}$ T. J. Khoo, ${ }^{28}$ G. Khoriauli, ${ }^{21}$ A. Khoroshilov, ${ }^{176}$ V. Khovanskiy, ${ }^{96}$ E. Khramov ${ }^{64}$ J. Khubua, ${ }^{51 \mathrm{~b}}$ D. W. Kim, ${ }^{15}$ H. Kim, ${ }^{147 \mathrm{a}, 147 \mathrm{~b}}$ S. H. Kim, ${ }^{161}$ N. Kimura, ${ }^{172}$ O. Kind, ${ }^{16}$ B. T. King, ${ }^{73}$ M. King, ${ }^{66}$ R. S. B. King, ${ }^{119}$ S. B. King, ${ }^{169}$ J. Kirk, ${ }^{130}$ A. E. Kiryunin, ${ }^{100}$ T. Kishimoto, ${ }^{66}$ D. Kisielewska, ${ }^{38 a}$ T. Kitamura, ${ }^{66}$ T. Kittelmann, ${ }^{124}$ K. Kiuchi, ${ }^{161}$ E. Kladiva, ${ }^{145 b}$ M. Klein, ${ }^{73}$ U. Klein, ${ }^{73}$ K. Kleinknecht, ${ }^{82}$ P. Klimek, ${ }^{147 a, 147 b}$ A. Klimentov, ${ }^{25}$ R. Klingenberg, ${ }^{43}$ J. A. Klinger, ${ }^{83}$ E. B. Klinkby, ${ }^{36}$ T. Klioutchnikova, ${ }^{30}$ P. F. Klok, ${ }^{105}$ E.-E. Kluge, ${ }^{58 a}$ P. Kluit, ${ }^{106}$ S. Kluth, ${ }^{100}$ E. Kneringer,${ }^{61}$ E. B. F. G. Knoops,${ }^{84}$ A. Knue,${ }^{54}$ B. R. Ko, ${ }^{45}$ T. Kobayashi, ${ }^{156}$ M. Kobel,${ }^{44}$ M. Kocian, ${ }^{144}$ P. Kodys, ${ }^{128}$ S. Koenig, ${ }^{82}$ P. Koevesarki, ${ }^{21}$ T. Koffas, ${ }^{29}$ E. Koffeman, ${ }^{106}$ L. A. Kogan, ${ }^{119}$ S. Kohlmann, ${ }^{176}$ F. Kohn, ${ }^{54}$ Z. Kohout, ${ }^{127}$ T. Kohriki, ${ }^{65}$ T. Koi, ${ }^{144}$ H. Kolanoski ${ }^{16}{ }^{16}$. Koletsou, ${ }^{90 a}$ J. Koll ${ }^{89}$ A. A. Komar, ${ }^{95, a}$ Y. Komori, ${ }^{156}$ T. Kondo, ${ }^{65}$ K. Köneke, ${ }^{48}$ A. C. König, ${ }^{105}$ T. Kono, ${ }^{42, v}$ R. Konoplich, ${ }^{109, w}$ N. Konstantinidis, ${ }^{77}$ R. Kopeliansky, ${ }^{153}$ S. Koperny, ${ }^{38 a}$ L. Köpke, ${ }^{82}$ A. K. Kopp ${ }^{48}$ K. Korcyl, ${ }^{39}$ K. Kordas, ${ }^{155}$ A. Korn, ${ }^{46}$ A. A. Korol, ${ }^{108}$ I. Korolkov, ${ }^{12}$ E. V. Korolkova, ${ }^{140}$ V. A. Korotkov, ${ }^{129}$ O. Kortner, ${ }^{100}$ S. Kortner, ${ }^{100}$ V. V. Kostyukhin, ${ }^{21}$ S. Kotov, ${ }^{100}$ V. M. Kotov, ${ }^{64}$ A. Kotwal, ${ }^{45}$ C. Kourkoumelis, ${ }^{9}$ V. Kouskoura, ${ }^{155}$ A. Koutsman, ${ }^{160 a}$ R. Kowalewski, ${ }^{170}$ T.Z. Kowalski, ${ }^{38 a}$ W. Kozanecki, ${ }^{137}$ A. S. Kozhin, ${ }^{129}$ V. Kral, ${ }^{127}$ V. A. Kramarenko, ${ }^{98}$ G. Kramberger, ${ }^{74}$ M. W. Krasny, ${ }^{79}$ A. Krasznahorkay, ${ }^{109}$ J. K. Kraus, ${ }^{21}$ A. Kravchenko, ${ }^{25}$ S. Kreiss, ${ }^{109}$ J. Kretzschmar, ${ }^{73}$ K. Kreutzfeldt, ${ }^{52}$ N. Krieger, ${ }^{54}$ P. Krieger, ${ }^{159}$ K. Kroeninger, ${ }^{54}$ H. Kroha, ${ }^{100}$ J. Kroll, ${ }^{121}$ J. Kroseberg, ${ }^{21}$ J. Krstic, ${ }^{13 a}$ U. Kruchonak, ${ }^{64}$ H. Krüger, ${ }^{21}$ T. Kruker,${ }^{17}$ N. Krumnack, ${ }^{63}$ Z. V. Krumshteyn, ${ }^{64}$ A. Kruse, ${ }^{174}$ M. C. Kruse, ${ }^{45}$ M. Kruskal, ${ }^{22}$ T. Kubota ${ }^{87}$ S. Kuday,${ }^{4 a}$ S. Kuehn, ${ }^{48}$ A. Kugel, ${ }^{58 c}$ T. Kuhl,${ }^{42}$ V. Kukhtin, ${ }^{64}$ Y. Kulchitsky, ${ }^{91}$ S. Kuleshov, ${ }^{32 b}$ M. Kuna, ${ }^{133 a, 133 b}$ J. Kunkle, ${ }^{121}$ A. Kupco, ${ }^{126}$ H. Kurashige, ${ }^{66}$ M. Kurata, ${ }^{161}$ 
Y. A. Kurochkin, ${ }^{91}$ R. Kurumida,${ }^{66}$ V. Kus, ${ }^{126}$ E. S. Kuwertz, ${ }^{148}$ M. Kuze, ${ }^{158}$ J. Kvita, ${ }^{143}$ R. Kwee, ${ }^{16}$ A. La Rosa, ${ }^{49}$ L. La Rotonda, ${ }^{37 a, 37 b}$ L. Labarga,${ }^{81}$ S. Lablak, ${ }^{136 a}$ C. Lacasta, ${ }^{168}$ F. Lacava, ${ }^{133 a, 133 b}$ J. Lacey, ${ }^{29}$ H. Lacker, ${ }^{16}$ D. Lacour, ${ }^{79}$ V. R. Lacuesta, ${ }^{168}$ E. Ladygin, ${ }^{64}$ R. Lafaye, ${ }^{5}$ B. Laforge,${ }^{79}$ T. Lagouri, ${ }^{177}$ S. Lai, ${ }^{48}$ H. Laier,${ }^{58 a}$ E. Laisne, ${ }^{55}$ L. Lambourne, ${ }^{77}$ C. L. Lampen, ${ }^{7}$ W. Lampl, ${ }^{7}$ E. Lançon, ${ }^{137}$ U. Landgraf, ${ }^{48}$ M. P. J. Landon,${ }^{75}$ V. S. Lang, ${ }^{58 a}$ C. Lange, ${ }^{42}$ A. J. Lankford, ${ }^{164}$ F. Lanni, ${ }^{25}$ K. Lantzsch, ${ }^{30}$ A. Lanza, ${ }^{120 a}$ S. Laplace, ${ }^{79}$ C. Lapoire, ${ }^{21}$ J. F. Laporte, ${ }^{137}$ T. Lari, ${ }^{90 a}$ A. Larner, ${ }^{119}$ M. Lassnig, ${ }^{30}$ P. Laurelli, ${ }^{47}$ V. Lavorini,,${ }^{37 a, 37 b}$ W. Lavrijsen, ${ }^{15}$ P. Laycock, ${ }^{73}$ B. T. Le,${ }^{55}$ O. Le Dortz,${ }^{79}$ E. Le Guirriec,${ }^{84}$ E. Le Menedeu, ${ }^{12}$ T. LeCompte, ${ }^{6}$ F. Ledroit-Guillon, ${ }^{55}$ C. A. Lee, ${ }^{152}$ H. Lee, ${ }^{106}$ J. S. H. Lee, ${ }^{117}$ S. C. Lee, ${ }^{152}$ L. Lee,${ }^{177}$ G. Lefebvre, ${ }^{79}$ M. Lefebvre, ${ }^{170}$ M. Legendre,,${ }^{137}$ F. Legger, ${ }^{99}$ C. Leggett, ${ }^{15}$ A. Lehan, ${ }^{73}$ M. Lehmacher, ${ }^{21}$ G. Lehmann Miotto, ${ }^{30}$ A. G. Leister, ${ }^{177}$ M. A. L. Leite, ${ }^{24 \mathrm{~d}}$ R. Leitner, ${ }^{128}$ D. Lellouch, ${ }^{173}$ B. Lemmer, ${ }^{54}$ V. Lendermann, ${ }^{58 \mathrm{a}}$ K. J. C. Leney, ${ }^{146 \mathrm{c}}$ T. Lenz, ${ }^{106}$ G. Lenzen, ${ }^{176}$ B. Lenzi, ${ }^{30}$ R. Leone, ${ }^{7}$ K. Leonhardt, ${ }^{44}$ S. Leontsinis,${ }^{10}$ C. Leroy, ${ }^{94}$ J-R. Lessard, ${ }^{170}$ C. G. Lester,${ }^{28}$ C. M. Lester,${ }^{121}$ J. Levêque,${ }^{5}$ D. Levin, ${ }^{88}$ L. J. Levinson, ${ }^{173}$ A. Lewis, ${ }^{119}$ G. H. Lewis, ${ }^{109}$ A. M. Leyko, ${ }^{21}$ M. Leyton, ${ }^{16}$ B. Li, ${ }^{33 b, x}$ B. Li, ${ }^{84}$ H. Li,${ }^{149}$ H. L. Li, ${ }^{31}$ S. Li ${ }^{45}$ X. Li,${ }^{88}$ Z. Liang, ${ }^{119, y}$ H. Liao, ${ }^{34}$ B. Liberti, ${ }^{134 a}$ P. Lichard,${ }^{30}$ K. Lie,${ }^{166}$ J. Liebal, ${ }^{21}$ W. Liebig, ${ }^{14}$ C. Limbach, ${ }^{21}$ A. Limosani, ${ }^{87}$ M. Limper ${ }^{62}$ S. C. Lin, ${ }^{152, z}$ F. Linde, ${ }^{106}$ B. E. Lindquist, ${ }^{149}$ J. T. Linnemann, ${ }^{89}$ E. Lipeles, ${ }^{121}$ A. Lipniacka, ${ }^{14}$ M. Lisovyi, ${ }^{42}$ T. M. Liss,${ }^{166}$ D. Lissauer,${ }^{25}$ A. Lister, ${ }^{169}$ A. M. Litke, ${ }^{138}$ B. Liu, ${ }^{152}$ D. Liu, ${ }^{152}$ J. B. Liu, ${ }^{33 b}$ K. Liu, ${ }^{33 b, a a}$ L. Liu, ${ }^{88}$ M. Liu, ${ }^{45}$ M. Liu, ${ }^{33 b}$ Y. Liu, ${ }^{33 b}$ M. Livan, ${ }^{120 a, 120 b}$ S. S. A. Livermore, ${ }^{119}$ A. Lleres, ${ }^{55}$ J. Llorente Merino, ${ }^{81}$ S. L. Lloyd ${ }^{75}$ F. Lo Sterzo, ${ }^{133 a, 133 b}$ E. Lobodzinska, ${ }^{42}$ P. Loch, ${ }^{7}$ W. S. Lockman,${ }^{138}$ T. Loddenkoetter, ${ }^{21}$ F. K. Loebinger ${ }^{83}$ A. E. Loevschall-Jensen, ${ }^{36}$ A. Loginov, ${ }^{177}$ C. W. Loh, ${ }^{169}$ T. Lohse, ${ }^{16}$

K. Lohwasser, ${ }^{48}$ M. Lokajicek, ${ }^{126}$ V. P. Lombardo,${ }^{5}$ R. E. Long,${ }^{71}$ L. Lopes,${ }^{125 a}$ D. Lopez Mateos, ${ }^{57}$

B. Lopez Paredes, ${ }^{140}$ J. Lorenz,${ }^{99}$ N. Lorenzo Martinez, ${ }^{116}$ M. Losada, ${ }^{163}$ P. Loscutoff, ${ }^{15}$ M. J. Losty, ${ }^{160 a, a}$ X. Lou, ${ }^{41}$ A. Lounis, ${ }^{116}$ J. Love, ${ }^{6}$ P. A. Love, ${ }^{71}$ A. J. Lowe,${ }^{144, \mathrm{~h}}$ F. Lu, ${ }^{33 \mathrm{a}}$ H. J. Lubatti, ${ }^{139}$ C. Luci, ${ }^{133 a, 133 b}$ A. Lucotte ${ }^{55}$ D. Ludwig, ${ }^{42}$ I. Ludwig, ${ }^{48}$ J. Ludwig, ${ }^{48}$ F. Luehring, ${ }^{60}$ W. Lukas, ${ }^{61}$ L. Luminari, ${ }^{133 a}$ E. Lund, ${ }^{118}$ J. Lundberg, ${ }^{147 a}$, $147 \mathrm{~b}$ O. Lundberg, ${ }^{147 a, 147 b}$ B. Lund-Jensen, ${ }^{148}$ M. Lungwitz, ${ }^{82}$ D. Lynn, ${ }^{25}$ R. Lysak, ${ }^{126}$ E. Lytken, ${ }^{80}$ H. Ma, ${ }^{25}$ L. L. Ma ${ }^{33 d}$ G. Maccarrone, ${ }^{47}$ A. Macchiolo, ${ }^{100}$ B. Maček,${ }^{74}$ J. Machado Miguens, ${ }^{125 a}$ D. Macina,${ }^{30}$ R. Mackeprang, ${ }^{36}$ R. Madar, ${ }^{48}$ R. J. Madaras, ${ }^{15}$ H. J. Maddocks,${ }^{71}$ W. F. Mader ${ }^{44}$ A. Madsen, ${ }^{167}$ M. Maeno, ${ }^{8}$ T. Maeno, ${ }^{25}$ L. Magnoni, ${ }^{164}$ E. Magradze,${ }^{54}$ K. Mahboubi, ${ }^{48}$ J. Mahlstedt, ${ }^{106}$ S. Mahmoud ${ }^{73}$ G. Mahout, ${ }^{18}$ C. Maiani, ${ }^{137}$ C. Maidantchik, ${ }^{24 a}$ A. Maio, ${ }^{125 a, e}$ S. Majewski, ${ }^{115}$ Y. Makida, ${ }^{65}$ N. Makovec,,${ }^{116}$ P. Mal, ${ }^{137, b b}$ B. Malaescu, ${ }^{79}$ Pa. Malecki, ${ }^{39}$ V. P. Maleev, ${ }^{122}$ F. Malek, ${ }^{55}$ U. Mallik, ${ }^{62}$ D. Malon, ${ }^{6}$ C. Malone,${ }^{144}$ S. Maltezos, ${ }^{10}$ V. M. Malyshev,${ }^{108}$ S. Malyukov, ${ }^{30}$ J. Mamuzic, ${ }^{13 \mathrm{~b}}$ L. Mandelli, ${ }^{90 \mathrm{a}}$ I. Mandić, ${ }^{74}$ R. Mandrysch, ${ }^{62}$ J. Maneira, ${ }^{125 a}$ A. Manfredini, ${ }^{100}$ L. Manhaes de Andrade Filho, ${ }^{24 b}$ J. A. Manjarres Ramos, ${ }^{137}$ A. Mann, ${ }^{99}$ P. M. Manning, ${ }^{138}$

A. Manousakis-Katsikakis, ${ }^{9}$ B. Mansoulie, ${ }^{137}$ R. Mantifel,${ }^{86}$ L. Mapelli, ${ }^{30}$ L. March, ${ }^{168}$ J. F. Marchand,${ }^{29}$ F. Marchese, ${ }^{134 a, 134 b}$ G. Marchiori, ${ }^{79}$ M. Marcisovsky, ${ }^{126}$ C.P. Marino, ${ }^{170}$ C. N. Marques, ${ }^{125 a}$ F. Marroquim,${ }^{24 a}$ Z. Marshall, ${ }^{15}$ L.F. Marti, ${ }^{17}$ S. Marti-Garcia, ${ }^{168}$ B. Martin, ${ }^{30}$ B. Martin, ${ }^{89}$ J. P. Martin, ${ }^{94}$ T. A. Martin, ${ }^{171}$ V. J. Martin, ${ }^{46}$ B. Martin dit Latour, ${ }^{49}$ H. Martinez, ${ }^{137}$ M. Martinez, ${ }^{12, s}$ S. Martin-Haugh, ${ }^{150}$ A. C. Martyniuk, ${ }^{170}$ M. Marx, ${ }^{139}$ F. Marzano, ${ }^{133 a}$ A. Marzin,,${ }^{112}$ L. Masetti, ${ }^{82}$ T. Mashimo, ${ }^{156}$ R. Mashinistov, ${ }^{95}$ J. Masik, ${ }^{83}$ A. L. Maslennikov, ${ }^{108}$ I. Massa, ${ }^{20 a, 20 b}$ N. Massol, ${ }^{5}$ P. Mastrandrea, ${ }^{149}$ A. Mastroberardino, ${ }^{37 a, 37 b}$ T. Masubuchi, ${ }^{156}$ H. Matsunaga ${ }^{156}$ T. Matsushita, ${ }^{66}$ P. Mättig, ${ }^{176}$ S. Mättig, ${ }^{42}$ J. Mattmann,${ }^{82}$ C. Mattravers, ${ }^{119, f}$ J. Maurer ${ }^{84}$ S. J. Maxfield, ${ }^{73}$ D. A. Maximov, ${ }^{108, i}$ R. Mazini, ${ }^{152}$ L. Mazzaferro, ${ }^{134 a, 134 b}$ M. Mazzanti,${ }^{90 a}$ G. Mc Goldrick, ${ }^{159}$ S. P. Mc Kee, ${ }^{88}$ A. McCarn, ${ }^{166}$ R. L. McCarthy, ${ }^{149}$ T. G. McCarthy, ${ }^{29}$ N. A. McCubbin, ${ }^{130}$ K. W. McFarlane, ${ }^{56, a}$ J. A. Mcfayden, ${ }^{140}$ G. Mchedlidze, ${ }^{51 b}$ T. Mclaughlan, ${ }^{18}$ S. J. McMahon, ${ }^{130}$ R. A. McPherson, ${ }^{170,1}$ A. Meade, ${ }^{85}$ J. Mechnich, ${ }^{106}$ M. Mechtel, ${ }^{176}$ M. Medinnis, ${ }^{42}$ S. Meehan, ${ }^{31}$ R. Meera-Lebbai, ${ }^{112}$ S. Mehlhase,${ }^{36}$ A. Mehta, ${ }^{73}$

K. Meier, ${ }^{58 \mathrm{a}}$ C. Meineck, ${ }^{99}$ B. Meirose,${ }^{80}$ C. Melachrinos, ${ }^{31}$ B. R. Mellado Garcia, ${ }^{146 \mathrm{c}}$ F. Meloni, ${ }^{90 a}, 90 \mathrm{~b}$ L. Mendoza Navas, ${ }^{163}$ A. Mengarelli, ${ }^{20 a, 20 b}$ S. Menke, ${ }^{100}$ E. Meoni, ${ }^{162}$ K. M. Mercurio,${ }^{57}$ S. Mergelmeyer, ${ }^{21}$ N. Meric, ${ }^{137}$ P. Mermod, ${ }^{49}$ L. Merola, ${ }^{103 a, 103 b}$ C. Meroni, ${ }^{90 a}$ F. S. Merritt, ${ }^{31}$ H. Merritt, ${ }^{110}$ A. Messina,${ }^{30, c c}$ J. Metcalfe, ${ }^{25}$ A. S. Mete, ${ }^{164}$ C. Meyer, ${ }^{82}$ C. Meyer, ${ }^{31}$ J-P. Meyer, ${ }^{137}$ J. Meyer, ${ }^{30}$ J. Meyer, ${ }^{54}$ S. Michal, ${ }^{30}$ R. P. Middleton, ${ }^{130}$ S. Migas, ${ }^{73}$ L. Mijović, ${ }^{137}$ G. Mikenberg, ${ }^{173}$ M. Mikestikova, ${ }^{126}$ M. Mikuž, ${ }^{74}$ D. W. Miller, ${ }^{31}$ W. J. Mills, ${ }^{169}$ C. Mills, ${ }^{57}$ A. Milov, ${ }^{173}$ D. A. Milstead, ${ }^{147 a, 147 b}$ D. Milstein, ${ }^{173}$ A. A. Minaenko, ${ }^{129}$

M. Miñano Moya, ${ }^{168}$ I. A. Minashvili, ${ }^{64}$ A. I. Mincer, ${ }^{109}$ B. Mindur, ${ }^{38 a}$ M. Mineev, ${ }^{64}$ Y. Ming,,${ }^{174}$ L. M. Mir, ${ }^{12}$ G. Mirabelli, ${ }^{133 a}$ T. Mitani, ${ }^{172}$ J. Mitrevski, ${ }^{138}$ V. A. Mitsou, ${ }^{168}$ S. Mitsui, ${ }^{65}$ P. S. Miyagawa, ${ }^{140}$ J. U. Mjörnmark, ${ }^{80}$ T. Moa ${ }^{147 \mathrm{a}, 147 \mathrm{~b}}$ V. Moeller ${ }^{28}$ S. Mohapatra, ${ }^{149}$ W. Mohr, ${ }^{48}$ S. Molander, ${ }^{147 a, 147 \mathrm{~b}}$ R. Moles-Valls, ${ }^{168}$ A. Molfetas,${ }^{30}$ K. Mönig, ${ }^{42}$ C. Monini, ${ }^{55}$ J. Monk,${ }^{36}$ E. Monnier, ${ }^{84}$ J. Montejo Berlingen, ${ }^{12}$ F. Monticelli, ${ }^{70}$ S. Monzani,${ }^{20 a, 20 b}$ 
R. W. Moore, ${ }^{3}$ C. Mora Herrera, ${ }^{49}$ A. Moraes,${ }^{53}$ N. Morange,${ }^{62}$ J. Morel,${ }^{54}$ D. Moreno, ${ }^{82}$ M. Moreno Llácer, ${ }^{168}$ P. Morettini, ${ }^{50 a}$ M. Morgenstern, ${ }^{44}$ M. Morii, ${ }^{57}$ S. Moritz, ${ }^{82}$ A. K. Morley, ${ }^{148}$ G. Mornacchi, ${ }^{30}$ J. D. Morris, ${ }^{75}$ L. Morvaj, ${ }^{102}$ H. G. Moser, ${ }^{100}$ M. Mosidze, ${ }^{51 b}$ J. Moss, ${ }^{110}$ R. Mount,,${ }^{144}$ E. Mountricha, ${ }^{10, d d}$ S. V. Mouraviev, ${ }^{95, a}$ E. J. W. Moyse, ${ }^{85}$ R. D. Mudd, ${ }^{18}$ F. Mueller, ${ }^{58 a}$ J. Mueller, ${ }^{124}$ K. Mueller, ${ }^{21}$ T. Mueller, ${ }^{28}$ T. Mueller, ${ }^{82}$ D. Muenstermann, ${ }^{49}$ Y. Munwes, ${ }^{154}$ J. A. Murillo Quijada,${ }^{18}$ W. J. Murray, ${ }^{130}$ I. Mussche,${ }^{106}$ E. Musto,${ }^{153}$ A. G. Myagkov, ${ }^{129, \text { ee }}$ M. Myska, ${ }^{126}$ O. Nackenhorst,${ }^{54}$ J. Nadal,${ }^{12}$ K. Nagai,${ }^{61}$ R. Nagai, ${ }^{158}$ Y. Nagai, ${ }^{84}$ K. Nagano, ${ }^{65}$ A. Nagarkar, ${ }^{110}$ Y. Nagasaka, ${ }^{59}$ M. Nagel, ${ }^{100}$ A. M. Nairz ${ }^{30}$ Y. Nakahama, ${ }^{30}$ K. Nakamura, ${ }^{65}$ T. Nakamura, ${ }^{156}$ I. Nakano, ${ }^{111}$ H. Namasivayam, ${ }^{41}$ G. Nanava, ${ }^{21}$ A. Napier, ${ }^{162}$ R. Narayan, ${ }^{58 b}$ M. Nash, ${ }^{77, f}$ T. Nattermann, ${ }^{21}$ T. Naumann, ${ }^{42}$ G. Navarro, ${ }^{163}$ H. A. Neal, ${ }^{88}$ P. Yu. Nechaeva, ${ }^{95}$ T. J. Neep,${ }^{83}$ A. Negri, ${ }^{120 a, 120 b}$ G. Negri, ${ }^{30}$ M. Negrini, ${ }^{20 a}$ S. Nektarijevic, ${ }^{49}$ A. Nelson, ${ }^{164}$ T. K. Nelson, ${ }^{144}$ S. Nemecek,${ }^{126}$ P. Nemethy, ${ }^{109}$ A. A. Nepomuceno, ${ }^{24 a}$ M. Nessi, ${ }^{30, f f}$ M. S. Neubauer, ${ }^{166}$ M. Neumann, ${ }^{176}$ A. Neusiedl, ${ }^{82}$ R. M. Neves, ${ }^{109}$ P. Nevski, ${ }^{25}$ F. M. Newcomer, ${ }^{121}$ P. R. Newman, ${ }^{18}$ D. H. Nguyen, ${ }^{6}$ V. Nguyen Thi Hong,${ }^{137}$ R. B. Nickerson, ${ }^{119}$ R. Nicolaidou,${ }^{137}$ B. Nicquevert, ${ }^{30}$ J. Nielsen, ${ }^{138}$ N. Nikiforou, ${ }^{35}$ A. Nikiforov, ${ }^{16}$ V. Nikolaenko, ${ }^{129, \text { ee }}$ I. Nikolic-Audit, ${ }^{79}$ K. Nikolics, ${ }^{49}$

K. Nikolopoulos, ${ }^{18}$ P. Nilsson, ${ }^{8}$ Y. Ninomiya, ${ }^{156}$ A. Nisati, ${ }^{133 a}$ R. Nisius, ${ }^{100}$ T. Nobe,${ }^{158}$ L. Nodulman, ${ }^{6}$

M. Nomachi, ${ }^{117}$ I. Nomidis, ${ }^{155}$ S. Norberg, ${ }^{112}$ M. Nordberg, ${ }^{30}$ J. Novakova, ${ }^{128}$ M. Nozaki,${ }^{65}$ L. Nozka, ${ }^{114}$

K. Ntekas, ${ }^{10}$ A.-E. Nuncio-Quiroz, ${ }^{21}$ G. Nunes Hanninger, ${ }^{87}$ T. Nunnemann, ${ }^{99}$ E. Nurse, ${ }^{77}$ B. J. O’Brien, ${ }^{46}$ F. O'grady, ${ }^{7}$ D. C. O'Neil, ${ }^{143}$ V. O'Shea, ${ }^{53}$ L. B. Oakes, ${ }^{99}$ F. G. Oakham, ${ }^{29,9}$ H. Oberlack, ${ }^{100}$ J. Ocariz, ${ }^{79}$ A. Ochi, ${ }^{66}$ M. I. Ochoa, ${ }^{77}$ S. Oda,${ }^{69}$ S. Odaka, ${ }^{65}$ J. Odier,${ }^{84}$ H. Ogren, ${ }^{60}$ A. Oh, ${ }^{83}$ S. H. Oh, ${ }^{45}$ C. C. Ohm,${ }^{30}$ T. Ohshima, ${ }^{102}$ W. Okamura, ${ }^{117}$ H. Okawa ${ }^{25}$ Y. Okumura, ${ }^{31}$ T. Okuyama, ${ }^{156}$ A. Olariu, ${ }^{26 a}$ A. G. Olchevski, ${ }^{64}$ S. A. Olivares Pino, ${ }^{46}$

M. Oliveira, ${ }^{125 a, j}$ D. Oliveira Damazio, ${ }^{25}$ E. Oliver Garcia, ${ }^{168}$ D. Olivito, ${ }^{121}$ A. Olszewski, ${ }^{39}$ J. Olszowska, ${ }^{39}$

A. Onofre, ${ }^{125 a, g g}$ P. U. E. Onyisi, ${ }^{31, h h}$ C. J. Oram, ${ }^{160 a}$ M. J. Oreglia,${ }^{31}$ Y. Oren, ${ }^{154}$ D. Orestano, ${ }^{135 a, 135 b}$ N. Orlando, ${ }^{72 a, 72 b}$ C. Oropeza Barrera, ${ }^{53}$ R. S. Orr ${ }^{159}$ B. Osculati, ${ }^{50 a, 50 b}$ R. Ospanov, ${ }^{121}$ G. Otero y Garzon, ${ }^{27}$ H. Otono, ${ }^{69}$ J. P. Ottersbach, ${ }^{106}$ M. Ouchrif, ${ }^{136 d}$ E. A. Ouellette, ${ }^{170}$ F. Ould-Saada, ${ }^{118}$ A. Ouraou, ${ }^{137}$ K. P. Oussoren, ${ }^{106}$ Q. Ouyang, ${ }^{33 a}$ A. Ovcharova, ${ }^{15}$ M. Owen, ${ }^{83}$ S. Owen, ${ }^{140}$ V. E. Ozcan, ${ }^{19 a}$ N. Ozturk,${ }^{8}$ K. Pachal, ${ }^{119}$

A. Pacheco Pages, ${ }^{12}$ C. Padilla Aranda,${ }^{12}$ S. Pagan Griso,${ }^{15}$ E. Paganis, ${ }^{140}$ C. Pahl,${ }^{100}$ F. Paige, ${ }^{25}$ P. Pais, ${ }^{85}$ K. Pajchel, ${ }^{118}$ G. Palacino, ${ }^{160 \mathrm{~b}}$ S. Palestini, ${ }^{30}$ D. Pallin, ${ }^{34}$ A. Palma, ${ }^{125 a}$ J. D. Palmer, ${ }^{18}$ Y. B. Pan, ${ }^{174}$ E. Panagiotopoulou, ${ }^{10}$ J. G. Panduro Vazquez, ${ }^{76}$ P. Pani,${ }^{106}$ N. Panikashvili, ${ }^{88}$ S. Panitkin, ${ }^{25}$ D. Pantea,${ }^{26 a}$ A. Papadelis, ${ }^{147 a}$ Th. D. Papadopoulou, ${ }^{10}$ K. Papageorgiou, ${ }^{155, \mathrm{r}}$ A. Paramonov, ${ }^{6}$ D. Paredes Hernandez, ${ }^{34}$ M. A. Parker, ${ }^{28}$ F. Parodi,${ }^{50 a, 50 b}$ J. A. Parsons, ${ }^{35}$ U. Parzefall,${ }^{48}$ S. Pashapour, ${ }^{54}$ E. Pasqualucci, ${ }^{133 a}$ S. Passaggio, ${ }^{50 a}$ A. Passeri, ${ }^{135 a}$ F. Pastore, ${ }^{135 a, 135 b, a}$ Fr. Pastore, ${ }^{76}$ G. Pásztor ${ }^{49, \text { ii }}$ S. Pataraia,${ }^{176}$ N. D. Patel, ${ }^{151}$ J. R. Pater, ${ }^{83}$ S. Patricelli, ${ }^{103 a, 103 b}$ T. Pauly, ${ }^{30}$ J. Pearce, ${ }^{170}$ M. Pedersen, ${ }^{118}$ S. Pedraza Lopez, ${ }^{168}$ M. I. Pedraza Morales, ${ }^{174}$ S. V. Peleganchuk, ${ }^{108}$ D. Pelikan, ${ }^{167}$ H. Peng, ${ }^{33 b}$ B. Penning, ${ }^{31}$ A. Penson,${ }^{35}$ J. Penwell, ${ }^{60}$ D. V. Perepelitsa,${ }^{35}$ T. Perez Cavalcanti, ${ }^{42}$ E. Perez Codina, ${ }^{160 a}$ M. T. Pérez García-Estañ, ${ }^{168}$ V. Perez Reale,${ }^{35}$ L. Perini, ${ }^{90 a, 90 b}$ H. Pernegger, ${ }^{30}$ R. Perrino, ${ }^{72 a}$ V. D. Peshekhonov, ${ }^{64}$ K. Peters,${ }^{30}$ R. F. Y. Peters, ${ }^{54, j j}$ B. A. Petersen, ${ }^{30}$ J. Petersen,${ }^{30}$ T. C. Petersen, ${ }^{36}$ E. Petit, ${ }^{5}$ A. Petridis, ${ }^{147 a, 147 b}$ C. Petridou, ${ }^{155}$ E. Petrolo, ${ }^{133 a}$ F. Petrucci, ${ }^{135 a, 135 b}$ M. Petteni, ${ }^{143}$ R. Pezoa, ${ }^{32 \mathrm{~b}}$ P. W. Phillips, ${ }^{130}$ G. Piacquadio, ${ }^{144}$ E. Pianori, ${ }^{171}$ A. Picazio, ${ }^{49}$ E. Piccaro, ${ }^{75}$ M. Piccinini, ${ }^{20 a, 20 b}$ S. M. Piec, ${ }^{42}$ R. Piegaia, ${ }^{27}$ D. T. Pignotti, ${ }^{110}$ J. E. Pilcher, ${ }^{31}$ A. D. Pilkington, ${ }^{77}$ J. Pina, ${ }^{125 a, e}$ M. Pinamonti, ${ }^{165 a, 165 c, k k}$ A. Pinder, ${ }^{119}$ J. L. Pinfold, ${ }^{3}$ A. Pingel, ${ }^{36}$ B. Pinto, ${ }^{125 a}$ C. Pizio, ${ }^{90 a, 90 b}$ M.-A. Pleier, ${ }^{25}$ V. Pleskot, ${ }^{128}$ E. Plotnikova, ${ }^{64}$ P. Plucinski, ${ }^{147 \mathrm{a}, 147 \mathrm{~b}}$ S. Poddar, ${ }^{58 \mathrm{a}}$ F. Podlyski, ${ }^{34}$ R. Poettgen,${ }^{82}$ L. Poggioli,,${ }^{116}$ D. Pohl,${ }^{21}$ M. Pohl,${ }^{49}$ G. Polesello, ${ }^{120 a}$ A. Policicchio, ${ }^{37 a, 37 b}$ R. Polifka, ${ }^{159}$ A. Polini, ${ }^{20 a}$ C. S. Pollard, ${ }^{45}$ V. Polychronakos, ${ }^{25}$ D. Pomeroy, ${ }^{23}$ K. Pommès, ${ }^{30}$

L. Pontecorvo, ${ }^{133 a}$ B. G. Pope ${ }^{89}$ G. A. Popeneciu, ${ }^{26 b}$ D. S. Popovic, ${ }^{13 a}$ A. Poppleton, ${ }^{30}$ X. Portell Bueso, ${ }^{12}$ G. E. Pospelov, ${ }^{100}$ S. Pospisil, ${ }^{127}$ K. Potamianos, ${ }^{15}$ I. N. Potrap, ${ }^{64}$ C. J. Potter, ${ }^{150}$ C. T. Potter,${ }^{15}$ G. Poulard, ${ }^{30}$ J. Poveda, ${ }^{60}$ V. Pozdnyakov, ${ }^{64}$ R. Prabhu,${ }^{77}$ P. Pralavorio, ${ }^{84}$ A. Pranko, ${ }^{15}$ S. Prasad, ${ }^{30}$ R. Pravahan, ${ }^{8}$ S. Prell, ${ }^{63}$

D. Price, ${ }^{60}$ J. Price, ${ }^{73}$ L.E. Price, ${ }^{6}$ D. Prieur, ${ }^{124}$ M. Primavera, ${ }^{72 a}$ M. Proissl,${ }^{46}$ K. Prokofiev, ${ }^{109}$ F. Prokoshin,${ }^{32 b}$ E. Protopapadaki, ${ }^{137}$ S. Protopopescu, ${ }^{25}$ J. Proudfoot,${ }^{6}$ X. Prudent,${ }^{44}$ M. Przybycien, ${ }^{38 a}$ H. Przysiezniak,${ }^{5}$ S. Psoroulas, ${ }^{21}$ E. Ptacek, ${ }^{115}$ E. Pueschel,${ }^{85}$ D. Puldon, ${ }^{149}$ M. Purohit ${ }^{25,11}$ P. Puzo, ${ }^{116}$ Y. Pylypchenko, ${ }^{62}$ J. Qian, ${ }^{88}$ A. Quadt, ${ }^{54}$ D. R. Quarrie, ${ }^{15}$ W. B. Quayle, ${ }^{146 \mathrm{c}}$ D. Quilty, ${ }^{53}$ V. Radeka,${ }^{25}$ V. Radescu, ${ }^{42}$ P. Radloff, ${ }^{115}$ F. Ragusa, ${ }^{90 a, 90 b}$ G. Rahal, ${ }^{179}$ S. Rajagopalan, ${ }^{25}$ M. Rammensee, ${ }^{48}$ M. Rammes, ${ }^{142}$ A. S. Randle-Conde, ${ }^{40}$ C. Rangel-Smith, ${ }^{79}$ K. Rao, ${ }^{164}$ F. Rauscher, ${ }^{99}$ T. C. Rave, ${ }^{48}$ T. Ravenscroft, ${ }^{53}$ M. Raymond, ${ }^{30}$ A. L. Read, ${ }^{118}$ D. M. Rebuzzi, ${ }^{120 a, 120 b}$ A. Redelbach, ${ }^{175}$ G. Redlinger ${ }^{25}$ R. Reece, ${ }^{121}$ K. Reeves, ${ }^{41}$ A. Reinsch, ${ }^{115}$ I. Reisinger, ${ }^{43}$ M. Relich, ${ }^{164}$ C. Rembser, ${ }^{30}$ Z. L. Ren, ${ }^{152}$ A. Renaud,${ }^{116}$ M. Rescigno, ${ }^{133 a}$ S. Resconi,${ }^{90 a}$ B. Resende, ${ }^{137}$ P. Reznicek, ${ }^{99}$ 
R. Rezvani, ${ }^{94}$ R. Richter, ${ }^{100}$ E. Richter-Was, ${ }^{38 b}$ M. Ridel,${ }^{79}$ P. Rieck, ${ }^{16}$ M. Rijssenbeek, ${ }^{149}$ A. Rimoldi, ${ }^{120 a, 120 b}$ L. Rinaldi, ${ }^{20 \mathrm{a}}$ R. R. Rios, ${ }^{40}$ E. Ritsch, ${ }^{61}$ I. Riu, ${ }^{12}$ G. Rivoltella, ${ }^{90 a, 90 b}$ F. Rizatdinova, ${ }^{113}$ E. Rizvi,${ }^{75}$ S. H. Robertson, ${ }^{86,1}$ A. Robichaud-Veronneau, ${ }^{119}$ D. Robinson, ${ }^{28}$ J. E. M. Robinson, ${ }^{83}$ A. Robson, ${ }^{53}$ J. G. Rocha de Lima, ${ }^{107}$ C. Roda, ${ }^{123 a, 123 b}$ D. Roda Dos Santos, ${ }^{126}$ L. Rodrigues, ${ }^{30}$ A. Roe, ${ }^{54}$ S. Roe, ${ }^{30}$ O. Røhne, ${ }^{118}$ S. Rolli, ${ }^{162}$ A. Romaniouk, ${ }^{97}$ M. Romano, ${ }^{20 a, 20 b}$ G. Romeo, ${ }^{27}$ E. Romero Adam, ${ }^{168}$ N. Rompotis, ${ }^{139}$ L. Roos, ${ }^{79}$ E. Ros, ${ }^{168}$ S. Rosati, ${ }^{133 a}$ K. Rosbach,,${ }^{49}$ A. Rose, ${ }^{150}$ M. Rose, ${ }^{76}$ P. L. Rosendahl, ${ }^{14}$ O. Rosenthal, ${ }^{142}$ V. Rossetti, ${ }^{12}$ E. Rossi, ${ }^{103 a, 103 b}$ L. P. Rossi ${ }^{50 a}$ R. Rosten, ${ }^{139}$ M. Rotaru, ${ }^{26 a}$ I. Roth, ${ }^{173}$ J. Rothberg, ${ }^{139}$ D. Rousseau, ${ }^{116}$ C. R. Royon, ${ }^{137}$ A. Rozanov, ${ }^{84}$ Y. Rozen, ${ }^{153}$ X. Ruan, ${ }^{146 c}$ F. Rubbo, ${ }^{12}$ I. Rubinskiy, ${ }^{42}$ N. Ruckstuhl, ${ }^{106}$ V. I. Rud, ${ }^{98}$ C. Rudolph, ${ }^{44}$ M. S. Rudolph, ${ }^{159}$ F. Rühr, ${ }^{7}$ A. Ruiz-Martinez, ${ }^{63}$ L. Rumyantsev, ${ }^{64}$ Z. Rurikova, ${ }^{48}$ N. A. Rusakovich, ${ }^{64}$ A. Ruschke, ${ }^{99}$ J. P. Rutherfoord, ${ }^{7}$ N. Ruthmann, ${ }^{48}$ P. Ruzicka, ${ }^{126}$ Y. F. Ryabov, ${ }^{122}$ M. Rybar, ${ }^{128}$ G. Rybkin, ${ }^{116}$

N. C. Ryder, ${ }^{119}$ A. F. Saavedra, ${ }^{151}$ A. Saddique, ${ }^{3}$ I. Sadeh,,${ }^{154}$ H. F-W. Sadrozinski, ${ }^{138}$ R. Sadykov, ${ }^{64}$ F. Safai Tehrani, ${ }^{133 a}$ H. Sakamoto, ${ }^{156}$ Y. Sakurai, ${ }^{172}$ G. Salamanna ${ }^{75}$ A. Salamon, ${ }^{134 a}$ M. Saleem, ${ }^{112}$ D. Salek, ${ }^{30}$ D. Salihagic, ${ }^{100}$ A. Salnikov, ${ }^{144}$ J. Salt, ${ }^{168}$ B. M. Salvachua Ferrando, ${ }^{6}$ D. Salvatore,${ }^{37 a, 37 b}$ F. Salvatore,${ }^{150}$ A. Salvucci, ${ }^{105}$ A. Salzburger, ${ }^{30}$ D. Sampsonidis, ${ }^{155}$ A. Sanchez, ${ }^{103 a, 103 b}$ J. Sánchez, ${ }^{168}$ V. Sanchez Martinez, ${ }^{168}$ H. Sandaker, ${ }^{14}$ H. G. Sander, ${ }^{82}$ M. P. Sanders, ${ }^{99}$ M. Sandhoff, ${ }^{176}$ T. Sandoval, ${ }^{28}$ C. Sandoval, ${ }^{163}$ R. Sandstroem, ${ }^{100}$ D. P. C. Sankey, ${ }^{130}$ A. Sansoni, ${ }^{47}$ C. Santoni,${ }^{34}$ R. Santonico, ${ }^{134 a, 134 b}$ H. Santos, ${ }^{125 a}$ I. Santoyo Castillo, ${ }^{150}$

K. Sapp, ${ }^{124}$ A. Sapronov, ${ }^{64}$ J. G. Saraiva, ${ }^{125 a}$ E. Sarkisyan-Grinbaum, ${ }^{8}$ B. Sarrazin, ${ }^{21}$ F. Sarri, ${ }^{123 a, 123 b}$ G. Sartisohn, ${ }^{176}$ O. Sasaki, ${ }^{65}$ Y. Sasaki, ${ }^{156}$ N. Sasao, ${ }^{67}$ I. Satsounkevitch, ${ }^{91}$ G. Sauvage, ${ }^{5, a}$ E. Sauvan, ${ }^{5}$ J. B. Sauvan, ${ }^{116}$ P. Savard, ${ }^{159, \mathrm{~g}}$ V. Savinov, ${ }^{124}$ D. O. Savu, ${ }^{30}$ C. Sawyer, ${ }^{119}$ L. Sawyer, ${ }^{78, \mathrm{n}}$ D. H. Saxon, ${ }^{53}$ J. Saxon, ${ }^{121}$ C. Sbarra ${ }^{20 a}$ A. Sbrizzi, ${ }^{3}$ T. Scanlon, ${ }^{30}$ D. A. Scannicchio, ${ }^{164}$ M. Scarcella, ${ }^{151}$ J. Schaarschmidt, ${ }^{116}$ P. Schacht,${ }^{100}$ D. Schaefer, ${ }^{121}$ A. Schaelicke, ${ }^{46}$ S. Schaepe, ${ }^{21}$ S. Schaetzel, ${ }^{58 b}$ U. Schäfer, ${ }^{82}$ A. C. Schaffer, ${ }^{16}$ D. Schaile,${ }^{99}$ R. D. Schamberger, ${ }^{149}$ V. Scharf, ${ }^{58 a}$ V. A. Schegelsky, ${ }^{122}$ D. Scheirich ${ }^{88}$ M. Schernau, ${ }^{164}$ M. I. Scherzer, ${ }^{35}$ C. Schiavi, ${ }^{50 a, 50 b}$ J. Schieck, ${ }^{99}$ C. Schillo, ${ }^{48}$ M. Schioppa,${ }^{37 a, 37 b}$ S. Schlenker ${ }^{30}$ E. Schmidt ${ }^{48}$ K. Schmieden, ${ }^{30}$ C. Schmitt, ${ }^{82}$ C. Schmitt, ${ }^{99}$ S. Schmitt,,${ }^{58 b}$ B. Schneider, ${ }^{17}$ Y. J. Schnellbach, ${ }^{73}$ U. Schnoor, ${ }^{44}$ L. Schoeffel, ${ }^{137}$ A. Schoening, ${ }^{58 b}$ A. L. S. Schorlemmer, ${ }^{54}$ M. Schott, ${ }^{82}$ D. Schouten, ${ }^{160 a}$ J. Schovancova, ${ }^{25}$ M. Schram, ${ }^{86}$ S. Schramm, ${ }^{159}$ M. Schreyer, ${ }^{175}$ C. Schroeder, ${ }^{82}$ N. Schroer,${ }^{58 c}$ N. Schuh, ${ }^{82}$ M. J. Schultens, ${ }^{21}$

H.-C. Schultz-Coulon, ${ }^{58 a}$ H. Schulz, ${ }^{16}$ M. Schumacher, ${ }^{48}$ B. A. Schumm, ${ }^{138}$ Ph. Schune, ${ }^{137}$ A. Schwartzman, ${ }^{144}$ $\mathrm{Ph}$. Schwegler, ${ }^{100} \mathrm{Ph}$. Schwemling, ${ }^{137}$ R. Schwienhorst ${ }^{89}$ J. Schwindling, ${ }^{137}$ T. Schwindt, ${ }^{21}$ M. Schwoerer, ${ }^{5}$ F. G. Sciacca, ${ }^{17}$ E. Scifo, ${ }^{116}$ G. Sciolla, ${ }^{23}$ W. G. Scott,${ }^{130}$ F. Scutti, ${ }^{21}$ J. Searcy, ${ }^{88}$ G. Sedov, ${ }^{42}$ E. Sedykh, ${ }^{122}$ S. C. Seidel, ${ }^{104}$ A. Seiden, ${ }^{138}$ F. Seifert, ${ }^{44}$ J. M. Seixas, ${ }^{24 a}$ G. Sekhniaidze, ${ }^{103 a}$ S. J. Sekula, ${ }^{40}$ K. E. Selbach,${ }^{46}$ D. M. Seliverstov, ${ }^{122}$ G. Sellers, ${ }^{73}$ M. Seman, ${ }^{145 b}$ N. Semprini-Cesari, ${ }^{20 a, 20 b}$ C. Serfon, ${ }^{30}$ L. Serin, ${ }^{116}$ L. Serkin,${ }^{54}$ T. Serre, ${ }^{84}$ R. Seuster, ${ }^{160 a}$ H. Severini, ${ }^{112}$ F. Sforza, ${ }^{100}$ A. Sfyrla, ${ }^{30}$ E. Shabalina,${ }^{54}$ M. Shamim, ${ }^{115}$ L. Y. Shan, ${ }^{33 a}$ J. T. Shank, ${ }^{22}$ Q. T. Shao, ${ }^{87}$ M. Shapiro, ${ }^{15}$ P. B. Shatalov, ${ }^{96}$ K. Shaw, ${ }^{165 a, 165 c}$ P. Sherwood, ${ }^{77}$ S. Shimizu, ${ }^{66}$ M. Shimojima, ${ }^{101}$ T. Shin, ${ }^{56}$ M. Shiyakova, ${ }^{64}$ A. Shmeleva, ${ }^{95}$ M. J. Shochet,${ }^{31}$ D. Short, ${ }^{119}$ S. Shrestha, ${ }^{63}$ E. Shulga,${ }^{97}$ M. A. Shupe, ${ }^{7}$ S. Shushkevich, ${ }^{42}$ P. Sicho, ${ }^{126}$ D. Sidorov, ${ }^{113}$ A. Sidoti, ${ }^{133 a}$ F. Siegert, ${ }^{48}$ Dj. Sijacki, ${ }^{13 a}$ O. Silbert, ${ }^{173}$ J. Silva, ${ }^{125 a}$ Y. Silver, ${ }^{154}$ D. Silverstein, ${ }^{144}$ S. B. Silverstein, ${ }^{147 a}$ V. Simak, ${ }^{127}$ O. Simard, ${ }^{5}$ Lj. Simic, ${ }^{13 a}$ S. Simion, ${ }^{116}$

E. Simioni, ${ }^{82}$ B. Simmons, ${ }^{77}$ R. Simoniello, ${ }^{90 a, 90 b}$ M. Simonyan, ${ }^{36}$ P. Sinervo, ${ }^{159}$ N. B. Sinev, ${ }^{115}$ V. Sipica, ${ }^{142}$

G. Siragusa, ${ }^{175}$ A. Sircar, ${ }^{78}$ A. N. Sisakyan,${ }^{64, a}$ S. Yu. Sivoklokov, ${ }^{98}$ J. Sjölin, ${ }^{147 a, 147 b}$ T. B. Sjursen, ${ }^{14}$

L. A. Skinnari, ${ }^{15}$ H. P. Skottowe, ${ }^{57}$ K. Yu. Skovpen, ${ }^{108}$ P. Skubic, ${ }^{112}$ M. Slater, ${ }^{18}$ T. Slavicek,${ }^{127}$ K. Sliwa, ${ }^{162}$ V. Smakhtin, ${ }^{173}$ B. H. Smart, ${ }^{46}$ L. Smestad, ${ }^{118}$ S. Yu. Smirnov, ${ }^{97}$ Y. Smirnov,${ }^{97}$ L. N. Smirnova, ${ }^{98, m m}$ O. Smirnova, ${ }^{80}$ K. M. Smith, ${ }^{53}$ M. Smizanska, ${ }^{71}$ K. Smolek, ${ }^{127}$ A. A. Snesarev,${ }^{95}$ G. Snidero, ${ }^{75}$ J. Snow, ${ }^{112}$ S. Snyder,${ }^{25}$ R. Sobie, ${ }^{170,1}$ J. Sodomka, ${ }^{127}$ A. Soffer, ${ }^{154}$ D. A. Soh, ${ }^{152, y}$ C. A. Solans, ${ }^{30}$ M. Solar, ${ }^{127}$ J. Solc, ${ }^{127}$ E. Yu. Soldatov, ${ }^{97}$ U. Soldevila, ${ }^{168}$

E. Solfaroli Camillocci, ${ }^{133 a, 133 b}$ A. A. Solodkov, ${ }^{129}$ O. V. Solovyanov, ${ }^{129}$ V. Solovyev, ${ }^{122}$ N. Soni, ${ }^{1}$ A. Sood,${ }^{15}$

V. Sopko, ${ }^{127}$ B. Sopko, ${ }^{127}$ M. Sosebee, ${ }^{8}$ R. Soualah, ${ }^{165 a, 165 c}$ P. Soueid, ${ }^{94}$ A. M. Soukharev, ${ }^{108}$ D. South, ${ }^{42}$

S. Spagnolo, ${ }^{72 a, 72 b}$ F. Spanò,${ }^{76}$ W. R. Spearman, ${ }^{57}$ R. Spighi, ${ }^{20 a}$ G. Spigo,${ }^{30}$ M. Spousta, ${ }^{128, n n}$ T. Spreitzer, ${ }^{159}$

B. Spurlock, ${ }^{8}$ R. D. St. Denis, ${ }^{53}$ J. Stahlman, ${ }^{121}$ R. Stamen, ${ }^{58 a}$ E. Stanecka, ${ }^{39}$ R. W. Stanek, ${ }^{6}$ C. Stanescu, ${ }^{135 a}$ M. Stanescu-Bellu, ${ }^{42}$ M. M. Stanitzki, ${ }^{42}$ S. Stapnes, ${ }^{118}$ E. A. Starchenko, ${ }^{129}$ J. Stark ${ }^{55}$ P. Staroba, ${ }^{126}$ P. Starovoitov, ${ }^{42}$ R. Staszewski, ${ }^{39}$ A. Staude, ${ }^{99}$ P. Stavina, ${ }^{145 a, a}$ G. Steele,${ }^{53}$ P. Steinbach, ${ }^{44}$ P. Steinberg, ${ }^{25}$ I. Stekl, ${ }^{127}$ B. Stelzer, ${ }^{143}$ H. J. Stelzer, ${ }^{89}$ O. Stelzer-Chilton, ${ }^{160 a}$ H. Stenzel, ${ }^{52}$ S. Stern, ${ }^{100}$ G. A. Stewart, ${ }^{30}$ J. A. Stillings, ${ }^{21}$ M. C. Stockton, ${ }^{86}$

M. Stoebe, ${ }^{86}$ K. Stoerig, ${ }^{48}$ G. Stoicea, ${ }^{26 a}$ S. Stonjek, ${ }^{100}$ A. R. Stradling, ${ }^{8}$ A. Straessner, ${ }^{44}$ J. Strandberg, ${ }^{148}$ S. Strandberg, ${ }^{147 a, 147 b}$ A. Strandlie, ${ }^{118}$ E. Strauss, ${ }^{144}$ M. Strauss, ${ }^{112}$ P. Strizenec, ${ }^{145 b}$ R. Ströhmer, ${ }^{175}$ D. M. Strom, ${ }^{115}$ 
R. Stroynowski, ${ }^{40}$ S. A. Stucci, ${ }^{17}$ B. Stugu, ${ }^{14}$ I. Stumer, ${ }^{25, a}$ J. Stupak, ${ }^{149}$ P. Sturm, ${ }^{176}$ N. A. Styles, ${ }^{42}$ D. Su,${ }^{144}$ HS. Subramania, ${ }^{3}$ R. Subramaniam,${ }^{78}$ A. Succurro, ${ }^{12}$ Y. Sugaya,${ }^{117}$ C. Suhr,${ }^{107}$ M. Suk, ${ }^{127}$ V. V. Sulin, ${ }^{95}$ S. Sultansoy, ${ }^{4 \mathrm{c}}$ T. Sumida, ${ }^{67}$ X. Sun,${ }^{55}$ J. E. Sundermann, ${ }^{48}$ K. Suruliz, ${ }^{140}$ G. Susinno, ${ }^{37 a, 37 b}$ M. R. Sutton, ${ }^{150}$ Y. Suzuki, ${ }^{65}$ M. Svatos, ${ }^{126}$ S. Swedish, ${ }^{169}$ M. Swiatlowski, ${ }^{144}$ I. Sykora, ${ }^{145 a}$ T. Sykora, ${ }^{128}$ D. Ta, ${ }^{106}$ K. Tackmann, ${ }^{42}$ J. Taenzer, ${ }^{159}$ A. Taffard,${ }^{164}$ R. Tafirout,${ }^{160 a}$ N. Taiblum, ${ }^{154}$ Y. Takahashi, ${ }^{102}$ H. Takai, ${ }^{25}$ R. Takashima,${ }^{68}$

H. Takeda ${ }^{66}$ T. Takeshita, ${ }^{141}$ Y. Takubo, ${ }^{65}$ M. Talby, ${ }^{84}$ A. A. Talyshev, ${ }^{108, i}$ J. Y. C. Tam, ${ }^{175}$ M. C. Tamsett, ${ }^{78, o o}$ K. G. Tan, ${ }^{87}$ J. Tanaka, ${ }^{156}$ R. Tanaka, ${ }^{116}$ S. Tanaka, ${ }^{132}$ S. Tanaka, ${ }^{65}$ A. J. Tanasijczuk, ${ }^{143}$ K. Tani, ${ }^{66}$ N. Tannoury, ${ }^{84}$ S. Tapprogge,${ }^{82}$ S. Tarem, ${ }^{153}$ F. Tarrade, ${ }^{29}$ G. F. Tartarelli, ${ }^{90 a}$ P. Tas, ${ }^{128}$ M. Tasevsky, ${ }^{126}$ T. Tashiro,${ }^{67}$ E. Tassi,${ }^{37 a, 37 b}$ A. Tavares Delgado, ${ }^{125 a}$ Y. Tayalati, ${ }^{136 \mathrm{~d}}$ C. Taylor ${ }^{77}$ F. E. Taylor, ${ }^{93}$ G. N. Taylor, ${ }^{87}$ W. Taylor,${ }^{160 b}$ F. A. Teischinger ${ }^{30}$ M. Teixeira Dias Castanheira, ${ }^{75}$ P. Teixeira-Dias, ${ }^{76}$ K. K. Temming,${ }^{48}$ H. Ten Kate,${ }^{30}$ P. K. Teng, ${ }^{152}$ S. Terada, ${ }^{65}$ K. Terashi, ${ }^{156}$ J. Terron, ${ }^{81}$ S. Terzo, ${ }^{100}$ M. Testa,${ }^{47}$ R. J. Teuscher, ${ }^{159,1}$ J. Therhaag, ${ }^{21}$ T. Theveneaux-Pelzer, ${ }^{34}$

S. Thoma, ${ }^{48}$ J. P. Thomas, ${ }^{18}$ E. N. Thompson, ${ }^{35}$ P. D. Thompson, ${ }^{18}$ P. D. Thompson, ${ }^{159}$ A. S. Thompson, ${ }^{53}$ L. A. Thomsen, ${ }^{36}$ E. Thomson, ${ }^{121}$ M. Thomson, ${ }^{28}$ W. M. Thong ${ }^{87}$ R. P. Thun,${ }^{88, a}$ F. Tian, ${ }^{35}$ M. J. Tibbetts, ${ }^{15}$ T. Tic, ${ }^{126}$ V. O. Tikhomirov, ${ }^{95}$ Yu. A. Tikhonov, ${ }^{108, i}$ S. Timoshenko, ${ }^{97}$ E. Tiouchichine,${ }^{84}$ P. Tipton, ${ }^{177}$ S. Tisserant,${ }^{84}$ T. Todorov, ${ }^{5}$ S. Todorova-Nova, ${ }^{128}$ B. Toggerson, ${ }^{164}$ J. Tojo, ${ }^{69}$ S. Tokár, ${ }^{145 a} \mathrm{~K}$. Tokushuku, ${ }^{65} \mathrm{~K}$. Tollefson,${ }^{89}$ L. Tomlinson, ${ }^{83}$ M. Tomoto, ${ }^{102}$ L. Tompkins,${ }^{31}$ K. Toms, ${ }^{104}$ A. Tonoyan,,${ }^{14}$ N. D. Topilin, ${ }^{64}$ E. Torrence,${ }^{15}$ H. Torres, ${ }^{143}$ E. Torró Pastor, ${ }^{168}$ J. Toth, ${ }^{84, \text { ii }}$ F. Touchard, ${ }^{84}$ D. R. Tovey,${ }^{140}$ H. L. Tran, ${ }^{116}$ T. Trefzger, ${ }^{175}$ L. Tremblet, ${ }^{30}$ A. Tricoli, ${ }^{30}$ I. M. Trigger, ${ }^{160 a}$ S. Trincaz-Duvoid, ${ }^{79}$ M. F. Tripiana, ${ }^{70}$ N. Triplett, ${ }^{25}$ W. Trischuk, ${ }^{159}$ B. Trocmé, ${ }^{55}$ C. Troncon, ${ }^{90 a}$ M. Trottier-McDonald, ${ }^{143}$ M. Trovatelli, ${ }^{135 a, 135 b}$ P. True ${ }^{89}$ M. Trzebinski, ${ }^{39}$ A. Trzupek,${ }^{39}$ C. Tsarouchas,${ }^{30}$ J. C-L. Tseng, ${ }^{119}$ P. V. Tsiareshka, ${ }^{91}$ D. Tsionou,,${ }^{137}$ G. Tsipolitis, ${ }^{10}$ S. Tsiskaridze, ${ }^{12}$ V. Tsiskaridze, ${ }^{48}$ E. G. Tskhadadze, ${ }^{51 \text { a }}$ I. I. Tsukerman, ${ }^{96}$ V. Tsulaia, ${ }^{15}$ J.-W. Tsung, ${ }^{21}$ S. Tsuno, ${ }^{65}$ D. Tsybychev, ${ }^{149}$ A. Tua, ${ }^{140}$ A. Tudorache, ${ }^{26 a}$ V. Tudorache, ${ }^{26 a}$ J. M. Tuggle,${ }^{31}$ A. N. Tuna, ${ }^{121}$ S. A. Tupputi, ${ }^{20 a, 20 b}$ S. Turchikhin,${ }^{98, m m}$ D. Turecek, ${ }^{127}$ I. Turk Cakir, ${ }^{4 \mathrm{~d}}$ R. Turra, ${ }^{90 a, 90 b}$ P. M. Tuts, ${ }^{35}$ A. Tykhonov, ${ }^{74}$ M. Tylmad,,${ }^{147 a, 147 b}$ M. Tyndel,,${ }^{130}$ K. Uchida ${ }^{21}$ I. Ueda, ${ }^{156}$ R. Ueno, ${ }^{29}$ M. Ughetto,${ }^{84}$ M. Ugland,,${ }^{14}$ M. Uhlenbrock, ${ }^{21}$ F. Ukegawa, ${ }^{161} \mathrm{G}$. Unal,${ }^{30}$ A. Undrus, ${ }^{25}$ G. Unel, ${ }^{164}$ F. C. Ungaro, ${ }^{48}$ Y. Unno, ${ }^{65}$ D. Urbaniec,,${ }^{35}$ P. Urquijo, ${ }^{21}$ G. Usai, ${ }^{8}$ A. Usanova, ${ }^{61}$ L. Vacavant ${ }^{84}$ V. Vacek,${ }^{127}$ B. Vachon,${ }^{86}$ S. Vahsen, ${ }^{15}$ N. Valencic, ${ }^{106}$ S. Valentinetti, ${ }^{20 a, 20 b}$ A. Valero, ${ }^{168}$ L. Valery, ${ }^{34}$ S. Valkar, ${ }^{128}$ E. Valladolid Gallego, ${ }^{168}$ S. Vallecorsa, ${ }^{49}$ J. A. Valls Ferrer, ${ }^{168}$ R. Van Berg, ${ }^{121}$ P. C. Van Der Deijl, ${ }^{106}$ R. van der Geer, ${ }^{106}$ H. van der Graaf, ${ }^{106}$ R. Van Der Leeuw, ${ }^{106}$ D. van der Ster, ${ }^{30}$ N. van Eldik, ${ }^{30}$ P. van Gemmeren, ${ }^{6}$ J. Van Nieuwkoop, ${ }^{143}$ I. van Vulpen, ${ }^{106}$ M. Vanadia, ${ }^{100}$ W. Vandelli, ${ }^{30}$ A. Vaniachine, ${ }^{6}$ P. Vankov, ${ }^{42}$ F. Vannucci ${ }^{79}$ R. Vari, ${ }^{133 a}$ E. W. Varnes, ${ }^{7}$ T. Varol, ${ }^{85}$ D. Varouchas, ${ }^{15}$ A. Vartapetian, ${ }^{8}$ K. E. Varvell, ${ }^{151}$ V. I. Vassilakopoulos, ${ }^{56}$ F. Vazeille, ${ }^{34}$ T. Vazquez Schroeder, ${ }^{54}$ J. Veatch, ${ }^{7}$ F. Veloso, ${ }^{125 a}$ S. Veneziano, ${ }^{133 a}$ A. Ventura, ${ }^{72 a, 72 b}$ D. Ventura, ${ }^{85}$

M. Venturi, ${ }^{48}$ N. Venturi, ${ }^{159}$ V. Vercesi,${ }^{120 a}$ M. Verducci, ${ }^{139}$ W. Verkerke, ${ }^{106}$ J. C. Vermeulen, ${ }^{106}$ A. Vest,${ }^{44}$ M. C. Vetterli, ${ }^{143, \mathrm{~g}}$ O. Viazlo, ${ }^{80}$ I. Vichou, ${ }^{166}$ T. Vickey, ${ }^{146 c, p p}$ O. E. Vickey Boeriu, ${ }^{146 c}$ G. H. A. Viehhauser, ${ }^{119}$ S. Viel, ${ }^{169}$ R. Vigne, ${ }^{30}$ M. Villa, ${ }^{20 a, 20 b}$ M. Villaplana Perez, ${ }^{168}$ E. Vilucchi,${ }^{47}$ M. G. Vincter, ${ }^{29}$ V. B. Vinogradov,${ }^{64}$ J. Virzi, ${ }^{15}$ O. Vitells, ${ }^{173}$ M. Viti, ${ }^{42}$ I. Vivarelli, ${ }^{48}$ F. Vives Vaque, ${ }^{3}$ S. Vlachos, ${ }^{10}$ D. Vladoiu, ${ }^{99}$ M. Vlasak, ${ }^{127}$ A. Vogel, ${ }^{21}$ P. Vokac, ${ }^{127}$ G. Volpi,${ }^{47}$ M. Volpi,${ }^{87}$ G. Volpini, ${ }^{90 a}$ H. von der Schmitt, ${ }^{100}$ H. von Radziewski,${ }^{48}$ E. von Toerne, ${ }^{21}$ V. Vorobel, ${ }^{128}$ M. Vos, ${ }^{168}$ R. Voss,${ }^{30}$ J. H. Vossebeld, ${ }^{73}$ N. Vranjes,${ }^{137}$ M. Vranjes Milosavljevic,,${ }^{106}$ V. Vrba, ${ }^{126}$ M. Vreeswijk, ${ }^{106}$ T. Vu Anh,${ }^{48}$ R. Vuillermet, ${ }^{30}$ I. Vukotic, ${ }^{31}$ Z. Vykydal, ${ }^{127}$ W. Wagner, ${ }^{176}$ P. Wagner,${ }^{21}$ S. Wahrmund, ${ }^{44}$ J. Wakabayashi, ${ }^{102}$ S. Walch, ${ }^{88}$ J. Walder ${ }^{71}$ R. Walker, ${ }^{99}$ W. Walkowiak, ${ }^{142}$ R. Wall, ${ }^{177}$ P. Waller, ${ }^{73}$ B. Walsh, ${ }^{177}$ C. Wang, ${ }^{45}$ H. Wang, ${ }^{174}$ H. Wang, ${ }^{40}$ J. Wang, ${ }^{152}$ J. Wang, ${ }^{33 a}$ K. Wang, ${ }^{86}$ R. Wang, ${ }^{104}$ S. M. Wang ${ }^{152}$ T. Wang ${ }^{21}$ X. Wang, ${ }^{177}$ A. Warburton, ${ }^{86}$ C.P. Ward ${ }^{28}$ D. R. Wardrope ${ }^{77}$ M. Warsinsky, ${ }^{48}$ A. Washbrook,${ }^{46}$ C. Wasicki, ${ }^{42}$ I. Watanabe, ${ }^{66}$ P. M. Watkins, ${ }^{18}$ A. T. Watson, ${ }^{18}$ I. J. Watson, ${ }^{151}$ M. F. Watson, ${ }^{18}$ G. Watts, ${ }^{139}$ S. Watts ${ }^{83}$ A. T. Waugh, ${ }^{151}$ B. M. Waugh ${ }^{77}$ S. Webb,${ }^{83}$ M. S. Weber, ${ }^{17}$ S. W. Weber, ${ }^{175}$ J. S. Webster, ${ }^{31}$ A. R. Weidberg, ${ }^{119}$ P. Weigell, ${ }^{100}$ J. Weingarten,${ }^{54}$ C. Weiser, ${ }^{48}$ H. Weits, ${ }^{106}$ P. S. Wells,${ }^{30}$ T. Wenaus, ${ }^{25}$ D. Wendland, ${ }^{16}$ Z. Weng, ${ }^{152, y}$ T. Wengler, ${ }^{30}$ S. Wenig, ${ }^{30}$ N. Wermes, ${ }^{21}$ M. Werner, ${ }^{48}$ P. Werner, ${ }^{30}$ M. Werth, ${ }^{164}$ M. Wessels, ${ }^{58 a}$ J. Wetter, ${ }^{162}$

K. Whalen, ${ }^{29}$ A. White, ${ }^{8}$ M. J. White, ${ }^{87}$ R. White, ${ }^{32 b}$ S. White, ${ }^{123 a, 123 b}$ D. Whiteson, ${ }^{164}$ D. Whittington, ${ }^{60}$

D. Wicke, ${ }^{176}$ F. J. Wickens, ${ }^{130}$ W. Wiedenmann, ${ }^{174}$ M. Wielers, ${ }^{80, f}$ P. Wienemann, ${ }^{21}$ C. Wiglesworth,${ }^{36}$

L. A. M. Wiik-Fuchs,${ }^{21}$ P. A. Wijeratne, ${ }^{77}$ A. Wildauer, ${ }^{100}$ M. A. Wildt, ${ }^{42, v}$ I. Wilhelm, ${ }^{128}$ H. G. Wilkens, ${ }^{30}$ J.Z. Will, ${ }^{99}$ E. Williams, ${ }^{35}$ H. H. Williams, ${ }^{121}$ S. Williams, ${ }^{28}$ W. Willis, ${ }^{35, a}$ S. Willocq,${ }^{85}$ J. A. Wilson, ${ }^{18}$ A. Wilson, ${ }^{88}$ I. Wingerter-Seez, ${ }^{5}$ S. Winkelmann, ${ }^{48}$ F. Winklmeier, ${ }^{30}$ M. Wittgen, ${ }^{144}$ T. Wittig, ${ }^{43}$ J. Wittkowski, ${ }^{99}$ S. J. Wollstadt ${ }^{82}$ M. W. Wolter, ${ }^{39}$ H. Wolters, ${ }^{125 a, j}$ W. C. Wong, ${ }^{41}$ G. Wooden, ${ }^{88}$ B. K. Wosiek, ${ }^{39}$ J. Wotschack, ${ }^{30}$ M. J. Woudstra, ${ }^{83}$ 
K. W. Wozniak, ${ }^{39}$ K. Wraight,${ }^{53}$ M. Wright,${ }^{53}$ B. Wrona, ${ }^{73}$ S. L. Wu, ${ }^{174}$ X. Wu, ${ }^{49}$ Y. Wu,${ }^{88}$ E. Wulf, ${ }^{35}$ T. R. Wyatt, ${ }^{83}$ B. M. Wynne, ${ }^{46}$ S. Xella, ${ }^{36}$ M. Xiao, ${ }^{137}$ C. Xu, ${ }^{33 b, d d}$ D. Xu, ${ }^{33 a}$ L. Xu,${ }^{33 b, q q}$ B. Yabsley,${ }^{151}$ S. Yacoob,,${ }^{146 b, r r}$ M. Yamada ${ }^{65}$ H. Yamaguchi, ${ }^{156}$ Y. Yamaguchi, ${ }^{156}$ A. Yamamoto, ${ }^{65}$ K. Yamamoto ${ }^{63}$ S. Yamamoto, ${ }^{156}$ T. Yamamura, ${ }^{156}$ T. Yamanaka, ${ }^{156}$ K. Yamauchi, ${ }^{102}$ Y. Yamazaki, ${ }^{66}$ Z. Yan, ${ }^{22}$ H. Yang, ${ }^{33 e}$ H. Yang, ${ }^{174}$ U. K. Yang, ${ }^{83}$ Y. Yang, ${ }^{110}$ Z. Yang, ${ }^{147 a, 147 b}$ S. Yanush, ${ }^{92}$ L. Yao, ${ }^{33 a}$ Y. Yasu, ${ }^{65}$ E. Yatsenko, ${ }^{42}$ K. H. Yau Wong, ${ }^{21}$ J. Ye, ${ }^{40}$ S. Ye,${ }^{25}$ A. L. Yen, ${ }^{57}$ E. Yildirim,${ }^{42}$ M. Yilmaz,${ }^{4 b}$ R. Yoosoofmiya,${ }^{124}$ K. Yorita, ${ }^{172}$ R. Yoshida,${ }^{6}$ K. Yoshihara, ${ }^{156}$ C. Young, ${ }^{144}$ C. J. S. Young, ${ }^{119}$ S. Youssef, ${ }^{22}$ D. R. Yu, ${ }^{15}$ J. Yu, ${ }^{8}$ J. Yu, ${ }^{113}$ L. Yuan, ${ }^{66}$

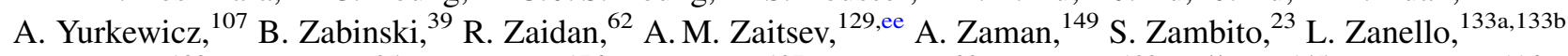
D. Zanzi, ${ }^{100}$ A. Zaytsev, ${ }^{25}$ C. Zeitnitz, ${ }^{176}$ M. Zeman, ${ }^{127}$ A. Zemla, ${ }^{39}$ O. Zenin, ${ }^{129}$ T. Ženišs, ${ }^{145 a}$ D. Zerwas, ${ }^{116}$ G. Zevi della Porta, ${ }^{57}$ D. Zhang, ${ }^{88}$ H. Zhang, ${ }^{89}$ J. Zhang, ${ }^{6}$ L. Zhang, ${ }^{152}$ X. Zhang, ${ }^{33 d}$ Z. Zhang, ${ }^{116}$ Z. Zhao, ${ }^{33 b}$ A. Zhemchugov, ${ }^{64}$ J. Zhong, ${ }^{119}$ B. Zhou ${ }^{88}$ L. Zhou, ${ }^{35}$ N. Zhou, ${ }^{164}$ C. G. Zhu, ${ }^{33 d}$ H. Zhu, ${ }^{42}$ J. Zhu, ${ }^{88}$ Y. Zhu, ${ }^{33 b}$ X. Zhuang, ${ }^{33 a}$ A. Zibell, ${ }^{99}$ D. Zieminska, ${ }^{60}$ N. I. Zimin, ${ }^{64}$ C. Zimmermann, ${ }^{82}$ R. Zimmermann, ${ }^{21}$ S. Zimmermann, ${ }^{21}$ S. Zimmermann, ${ }^{48}$ Z. Zinonos, ${ }^{123 a, 123 b}$ M. Ziolkowski, ${ }^{142}$ R. Zitoun, ${ }^{5}$ L. Živković, ${ }^{35}$ G. Zobernig, ${ }^{174}$ A. Zoccoli, ${ }^{20 a, 20 b}$ M. zur Nedden, ${ }^{16}$ G. Zurzolo, ${ }^{103 a, 103 b}$ V. Zutshi, ${ }^{107}$ and L. Zwalinski ${ }^{30}$

\title{
(ATLAS Collaboration)
}

\author{
${ }^{1}$ School of Chemistry and Physics, University of Adelaide, Adelaide, Australia \\ ${ }^{2}$ Physics Department, SUNY Albany, Albany, New York, USA \\ ${ }^{3}$ Department of Physics, University of Alberta, Edmonton, Alberta, Canada \\ ${ }^{4 a}$ Department of Physics, Ankara University, Ankara, Turkey \\ ${ }^{4 b}$ Department of Physics, Gazi University, Ankara, Turkey \\ ${ }^{4 \mathrm{c}}$ Division of Physics, TOBB University of Economics and Technology, Ankara, Turkey \\ ${ }^{4 \mathrm{~d}}$ Turkish Atomic Energy Authority, Ankara, Turkey \\ ${ }^{5}$ LAPP, CNRS/IN2P3 and Université de Savoie, Annecy-le-Vieux, France \\ ${ }^{6}$ High Energy Physics Division, Argonne National Laboratory, Argonne, Illinois, USA \\ ${ }^{7}$ Department of Physics, University of Arizona, Tucson, Arizona, USA \\ ${ }^{8}$ Department of Physics, The University of Texas at Arlington, Arlington, Texas, USA \\ ${ }^{9}$ Physics Department, University of Athens, Athens, Greece \\ ${ }^{10}$ Physics Department, National Technical University of Athens, Zografou, Greece \\ ${ }^{11}$ Institute of Physics, Azerbaijan Academy of Sciences, Baku, Azerbaijan \\ ${ }^{12}$ Institut de Física d'Altes Energies and Departament de Física de la Universitat Autònoma de Barcelona, Barcelona, Spain \\ ${ }^{13 a}$ Institute of Physics, University of Belgrade, Belgrade, Serbia \\ ${ }^{13 \mathrm{~b}}$ Vinca Institute of Nuclear Sciences, University of Belgrade, Belgrade, Serbia \\ ${ }^{14}$ Department for Physics and Technology, University of Bergen, Bergen, Norway \\ ${ }^{15}$ Physics Division, Lawrence Berkeley National Laboratory and University of California, Berkeley, California, USA \\ ${ }^{16}$ Department of Physics, Humboldt University, Berlin, Germany \\ ${ }^{17}$ Albert Einstein Center for Fundamental Physics and Laboratory for High Energy Physics, University of Bern, Bern, Switzerland \\ ${ }^{18}$ School of Physics and Astronomy, University of Birmingham, Birmingham, United Kingdom \\ ${ }^{19 a}$ Department of Physics, Bogazici University, Istanbul, Turkey \\ ${ }^{19 b}$ Department of Physics, Dogus University, Istanbul, Turkey \\ ${ }^{19 \mathrm{c}}$ Department of Physics Engineering, Gaziantep University, Gaziantep, Turkey \\ ${ }^{20 a}$ INFN Sezione di Bologna, Bologna, Italy \\ ${ }^{20 \mathrm{~b}}$ Dipartimento di Fisica e Astronomia, Università di Bologna, Bologna, Italy \\ ${ }^{21}$ Physikalisches Institut, University of Bonn, Bonn, Germany \\ ${ }^{22}$ Department of Physics, Boston University, Boston, Massachusetts, USA \\ ${ }^{23}$ Department of Physics, Brandeis University, Waltham, Massachusetts, USA \\ ${ }^{24 a}$ Universidade Federal do Rio De Janeiro COPPE/EE/IF, Rio de Janeiro, Brazil \\ ${ }^{24 \mathrm{~b}}$ Federal University of Juiz de Fora (UFJF), Juiz de Fora, Brazil \\ ${ }^{24 \mathrm{c}}$ Federal University of Sao Joao del Rei (UFSJ), Sao Joao del Rei, Brazil \\ ${ }^{24 \mathrm{~d}}$ Instituto de Fisica, Universidade de Sao Paulo, Sao Paulo, Brazil \\ ${ }^{25}$ Physics Department, Brookhaven National Laboratory, Upton, New York, USA \\ ${ }^{26 a}$ National Institute of Physics and Nuclear Engineering, Bucharest, Romania \\ ${ }^{26 \mathrm{~b}}$ National Institute for Research and Development of Isotopic and \\ Molecular Technologies, Physics Department, Cluj Napoca, Romania \\ ${ }^{26 \mathrm{c}}$ University Politehnica Bucharest, Bucharest, Romania \\ ${ }^{26 \mathrm{~d}}$ West University in Timisoara, Timisoara, Romania
}


${ }^{27}$ Departamento de Física, Universidad de Buenos Aires, Buenos Aires, Argentina

${ }^{28}$ Cavendish Laboratory, University of Cambridge, Cambridge, United Kingdom

${ }^{29}$ Department of Physics, Carleton University, Ottawa, Ontario, Canada

${ }^{30}$ CERN, Geneva, Switzerland

${ }^{31}$ Enrico Fermi Institute, University of Chicago, Chicago, Illinois, USA

${ }^{32 a}$ Departamento de Física, Pontificia Universidad Católica de Chile, Santiago, Chile

${ }^{32 \mathrm{~b}}$ Departamento de Física, Universidad Técnica Federico Santa María, Valparaíso, Chile

${ }^{33 a}$ Institute of High Energy Physics, Chinese Academy of Sciences, Beijing, China

${ }^{33 \mathrm{~b}}$ Department of Modern Physics, University of Science and Technology of China, Anhui, China

${ }^{33 \mathrm{c}}$ Department of Physics, Nanjing University, Jiangsu, China

${ }^{33 \mathrm{~d}}$ School of Physics, Shandong University, Shandong, China

${ }^{33}$ Physics Department, Shanghai Jiao Tong University, Shanghai, China

${ }^{34}$ Laboratoire de Physique Corpusculaire, Clermont Université and Université Blaise Pascal and CNRS/IN2P3, Clermont-Ferrand, France

${ }^{35}$ Nevis Laboratory, Columbia University, Irvington, New York, USA

${ }^{36}$ Niels Bohr Institute, University of Copenhagen, Kobenhavn, Denmark

${ }^{37}$ INFN Gruppo Collegato di Cosenza, Rende, Italy

${ }^{37 \mathrm{~b}}$ Dipartimento di Fisica, Università della Calabria, Rende, Italy

${ }^{38 \mathrm{a}}$ AGH University of Science and Technology, Faculty of Physics and Applied Computer Science, Krakow, Poland

${ }^{38 \mathrm{~b}}$ Marian Smoluchowski Institute of Physics, Jagiellonian University, Krakow, Poland

${ }^{39}$ The Henryk Niewodniczanski Institute of Nuclear Physics, Polish Academy of Sciences, Krakow, Poland

${ }^{40}$ Physics Department, Southern Methodist University, Dallas, Texas, USA

${ }^{41}$ Physics Department, University of Texas at Dallas, Richardson, Texas, USA

${ }^{42}$ DESY, Hamburg and Zeuthen, Germany

${ }^{43}$ Institut für Experimentelle Physik IV, Technische Universität Dortmund, Dortmund, Germany

${ }^{44}$ Institut für Kern- und Teilchenphysik, Technische Universität Dresden, Dresden, Germany

${ }^{45}$ Department of Physics, Duke University, Durham, North Carolina, USA

${ }^{46}$ SUPA - School of Physics and Astronomy, University of Edinburgh, Edinburgh, United Kingdom

${ }^{47}$ INFN Laboratori Nazionali di Frascati, Frascati, Italy

${ }^{48}$ Fakultät für Mathematik und Physik, Albert-Ludwigs-Universität, Freiburg, Germany

${ }^{49}$ Section de Physique, Université de Genève, Geneva, Switzerland

${ }^{50 a}$ INFN Sezione di Genova, Genova, Italy

${ }^{50 \mathrm{~b}}$ Dipartimento di Fisica, Università di Genova, Genova, Italy

${ }^{51 \mathrm{a}}$ E. Andronikashvili Institute of Physics, Iv. Javakhishvili Tbilisi State University, Tbilisi, Georgia

${ }^{51 \mathrm{~b}}$ High Energy Physics Institute, Tbilisi State University, Tbilisi, Georgia

${ }^{52}$ II Physikalisches Institut, Justus-Liebig-Universität Giessen, Giessen, Germany

${ }^{53}$ SUPA - School of Physics and Astronomy, University of Glasgow, Glasgow, United Kingdom

${ }^{54}$ II Physikalisches Institut, Georg-August-Universität, Göttingen, Germany

${ }^{55}$ Laboratoire de Physique Subatomique et de Cosmologie, Université Joseph Fourier and CNRS/IN2P3 and Institut National Polytechnique de Grenoble, Grenoble, France

${ }^{56}$ Department of Physics, Hampton University, Hampton, Virginia, USA

${ }^{57}$ Laboratory for Particle Physics and Cosmology, Harvard University, Cambridge, Massachusetts, USA

${ }^{58}$ Kirchhoff-Institut für Physik, Ruprecht-Karls-Universität Heidelberg, Heidelberg, Germany

${ }^{58 b}$ Physikalisches Institut, Ruprecht-Karls-Universität Heidelberg, Heidelberg, Germany

${ }^{58 \mathrm{c}}$ ZITI Institut für technische Informatik, Ruprecht-Karls-Universität Heidelberg, Mannheim, Germany

${ }^{59}$ Faculty of Applied Information Science, Hiroshima Institute of Technology, Hiroshima, Japan

${ }^{60}$ Department of Physics, Indiana University, Bloomington, Indiana, USA

${ }^{61}$ Institut für Astro- und Teilchenphysik, Leopold-Franzens-Universität, Innsbruck, Austria

${ }^{62}$ University of Iowa, Iowa City, Iowa, USA

${ }^{63}$ Department of Physics and Astronomy, Iowa State University, Ames, Iowa, USA

${ }^{64}$ Joint Institute for Nuclear Research, JINR Dubna, Dubna, Russia

${ }^{65}$ KEK, High Energy Accelerator Research Organization, Tsukuba, Japan

${ }^{66}$ Graduate School of Science, Kobe University, Kobe, Japan

${ }^{67}$ Faculty of Science, Kyoto University, Kyoto, Japan

${ }^{68}$ Kyoto University of Education, Kyoto, Japan

${ }^{69}$ Department of Physics, Kyushu University, Fukuoka, Japan

${ }^{70}$ Instituto de Física La Plata, Universidad Nacional de La Plata and CONICET, La Plata, Argentina

${ }^{71}$ Physics Department, Lancaster University, Lancaster, United Kingdom

${ }^{72 a}$ INFN Sezione di Lecce, Lecce, Italy

${ }^{72 b}$ Dipartimento di Matematica e Fisica, Università del Salento, Lecce, Italy

${ }^{73}$ Oliver Lodge Laboratory, University of Liverpool, Liverpool, United Kingdom 
${ }^{74}$ Department of Physics, Jožef Stefan Institute and University of Ljubljana, Ljubljana, Slovenia

${ }^{75}$ School of Physics and Astronomy, Queen Mary University of London, London, United Kingdom

${ }^{76}$ Department of Physics, Royal Holloway University of London, Surrey, United Kingdom

${ }^{77}$ Department of Physics and Astronomy, University College London, London, United Kingdom

${ }^{78}$ Louisiana Tech University, Ruston, Louisiana, USA

${ }^{79}$ Laboratoire de Physique Nucléaire et de Hautes Energies, UPMC and Université Paris-Diderot and CNRS/IN2P3, Paris, France

${ }^{80}$ Fysiska institutionen, Lunds universitet, Lund, Sweden

${ }^{81}$ Departamento de Fisica Teorica C-15, Universidad Autonoma de Madrid, Madrid, Spain

${ }^{82}$ Institut für Physik, Universität Mainz, Mainz, Germany

${ }^{83}$ School of Physics and Astronomy, University of Manchester, Manchester, United Kingdom

${ }^{84}$ CPPM, Aix-Marseille Université and CNRS/IN2P3, Marseille, France

${ }^{85}$ Department of Physics, University of Massachusetts, Amherst, Massachusetts, USA

${ }^{86}$ Department of Physics, McGill University, Montreal, Quebec City, Canada

${ }^{87}$ School of Physics, University of Melbourne, Victoria, Australia

${ }^{88}$ Department of Physics, The University of Michigan, Ann Arbor, Michigan, USA

${ }^{89}$ Department of Physics and Astronomy, Michigan State University, East Lansing, Michigan, USA

${ }^{90 a}$ INFN Sezione di Milano, Milano, Italy

${ }^{90 \mathrm{~b}}$ Dipartimento di Fisica, Università di Milano, Milano, Italy

${ }^{91}$ B.I. Stepanov Institute of Physics, National Academy of Sciences of Belarus, Minsk, Republic of Belarus

${ }^{92}$ National Scientific and Educational Centre for Particle and High Energy Physics, Minsk, Republic of Belarus

${ }^{93}$ Department of Physics, Massachusetts Institute of Technology, Cambridge, Massachusetts, USA

${ }^{94}$ Group of Particle Physics, University of Montreal, Montreal, Quebec City, Canada

${ }^{95}$ P.N. Lebedev Institute of Physics, Academy of Sciences, Moscow, Russia

${ }^{96}$ Institute for Theoretical and Experimental Physics (ITEP), Moscow, Russia

${ }^{97}$ Moscow Engineering and Physics Institute (MEPhI), Moscow, Russia

${ }^{98}$ D.V. Skobeltsyn Institute of Nuclear Physics, M.V. Lomonosov Moscow State University, Moscow, Russia

${ }^{99}$ Fakultät für Physik, Ludwig-Maximilians-Universität München, München, Germany

${ }^{100}$ Max-Planck-Institut für Physik (Werner-Heisenberg-Institut), München, Germany

${ }^{101}$ Nagasaki Institute of Applied Science, Nagasaki, Japan

${ }^{102}$ Graduate School of Science and Kobayashi-Maskawa Institute, Nagoya University, Nagoya, Japan

${ }^{103 a}$ INFN Sezione di Napoli, Napoli, Italy

${ }^{103 \mathrm{~b}}$ Dipartimento di Scienze Fisiche, Università di Napoli, Napoli, Italy

${ }^{104}$ Department of Physics and Astronomy, University of New Mexico, Albuquerque, New Mexico, USA

${ }^{105}$ Institute for Mathematics, Astrophysics and Particle Physics, Radboud University Nijmegen/Nikhef, Nijmegen, Netherlands

${ }^{106}$ Nikhef National Institute for Subatomic Physics and University of Amsterdam, Amsterdam, Netherlands

${ }^{107}$ Department of Physics, Northern Illinois University, DeKalb, Illinois, USA

${ }^{108}$ Budker Institute of Nuclear Physics, SB RAS, Novosibirsk, Russia

${ }^{109}$ Department of Physics, New York University, New York, New York, USA

${ }^{110}$ Ohio State University, Columbus, Ohio, USA

${ }^{111}$ Faculty of Science, Okayama University, Okayama, Japan

${ }^{112}$ Homer L. Dodge Department of Physics and Astronomy, University of Oklahoma, Norman, Oklahoma, USA

${ }^{113}$ Department of Physics, Oklahoma State University, Stillwater, Oklahoma, USA

${ }^{114}$ Palacký University, RCPTM, Olomouc, Czech Republic

${ }^{115}$ Center for High Energy Physics, University of Oregon, Eugene, Oregon, USA

${ }^{116}$ LAL, Université Paris-Sud and CNRS/IN2P3, Orsay, France

${ }^{117}$ Graduate School of Science, Osaka University, Osaka, Japan

${ }^{118}$ Department of Physics, University of Oslo, Oslo, Norway

${ }^{119}$ Department of Physics, Oxford University, Oxford, United Kingdom

${ }^{120 a}$ INFN Sezione di Pavia, Pavia, Italy

${ }^{120 \mathrm{~b}}$ Dipartimento di Fisica, Università di Pavia, Pavia, Italy

${ }^{121}$ Department of Physics, University of Pennsylvania, Philadelphia, Pennsylvania, USA

${ }^{122}$ Petersburg Nuclear Physics Institute, Gatchina, Russia

${ }^{123}$ INFN Sezione di Pisa, Pisa, Italy

${ }^{123 \mathrm{~b}}$ Dipartimento di Fisica E. Fermi, Università di Pisa, Pisa, Italy

${ }^{124}$ Department of Physics and Astronomy, University of Pittsburgh, Pittsburgh, Pennsylvania, USA

${ }^{125 a}$ Laboratorio de Instrumentacao e Fisica Experimental de Particulas-LIP, Lisboa, Portugal

${ }^{125 \mathrm{~b}}$ Departamento de Fisica Teorica y del Cosmos and CAFPE, Universidad de Granada, Granada, Spain

${ }^{126}$ Institute of Physics, Academy of Sciences of the Czech Republic, Praha, Czech Republic

${ }^{127}$ Czech Technical University in Prague, Praha, Czech Republic

${ }^{128}$ Faculty of Mathematics and Physics, Charles University in Prague, Praha, Czech Republic

${ }^{129}$ State Research Center Institute for High Energy Physics, Protvino, Russia 
${ }^{130}$ Particle Physics Department, Rutherford Appleton Laboratory, Didcot, United Kingdom

${ }^{131}$ Physics Department, University of Regina, Regina, Saskatchewan, Canada

${ }^{132}$ Ritsumeikan University, Kusatsu, Shiga, Japan

${ }^{133 a}$ INFN Sezione di Roma I, Italy

${ }^{133 b}$ Dipartimento di Fisica, Università La Sapienza, Roma, Italy

${ }^{134 a}$ INFN Sezione di Roma Tor Vergata, Italy

${ }^{134 \mathrm{~b}}$ Dipartimento di Fisica, Università di Roma Tor Vergata, Roma, Italy

${ }^{135 a}$ INFN Sezione di Roma Tre, Roma, Italy

${ }^{135 b}$ Dipartimento di Matematica e Fisica, Università Roma Tre, Roma, Italy

${ }^{136 a}$ Faculté des Sciences Ain Chock, Réseau Universitaire de Physique des Hautes Energies-Université Hassan II,

Casablanca, Morocco

${ }^{136 \mathrm{~b}}$ Centre National de l'Energie des Sciences Techniques Nucleaires, Rabat, Morocco

${ }^{136 c}$ Faculté des Sciences Semlalia, Université Cadi Ayyad, LPHEA-Marrakech, Morocco

${ }^{136 \mathrm{~d}}$ Faculté des Sciences, Université Mohamed Premier and LPTPM, Oujda, Morocco

${ }^{136 \mathrm{e}}$ Faculté des sciences, Université Mohammed V-Agdal, Rabat, Morocco

${ }^{137}$ DSM/IRFU (Institut de Recherches sur les Lois Fondamentales de l'Univers), CEA Saclay (Commissariat à l'Energie Atomique et aux Energies Alternatives), Gif-sur-Yvette, France

${ }^{138}$ Santa Cruz. Institute for Particle Physics, University of California Santa Cruz, Santa Cruz, California, USA

${ }^{139}$ Department of Physics, University of Washington, Seattle, Washington, USA

${ }^{140}$ Department of Physics and Astronomy, University of Sheffield, Sheffield, United Kingdom

${ }^{141}$ Department of Physics, Shinshu University, Nagano, Japan

${ }^{142}$ Fachbereich Physik, Universität Siegen, Siegen, Germany

${ }^{143}$ Department of Physics, Simon Fraser University, Burnaby, British Columbia, Canada

${ }^{144}$ SLAC National Accelerator Laboratory, Stanford, California, USA

${ }^{145 a}$ Faculty of Mathematics, Physics \& Informatics, Comenius University, Bratislava, Slovak Republic

${ }^{145 \mathrm{~b}}$ Department of Subnuclear Physics, Institute of Experimental Physics of the Slovak Academy of Sciences, Kosice, Slovak Republic

${ }^{146 a}$ Department of Physics, University of Cape Town, Cape Town, South Africa

${ }^{146 \mathrm{~b}}$ Department of Physics, University of Johannesburg, Johannesburg, South Africa

${ }^{146 c}$ School of Physics, University of the Witwatersrand, Johannesburg, South Africa

${ }^{147}$ Department of Physics, Stockholm University, Stockholm, Sweden

${ }^{147 \mathrm{~b}}$ The Oskar Klein Centre, Stockholm, Sweden

${ }^{148}$ Physics Department, Royal Institute of Technology, Stockholm, Sweden

${ }^{149}$ Departments of Physics \& Astronomy and Chemistry, Stony Brook University, Stony Brook, New York, USA

${ }^{150}$ Department of Physics and Astronomy, University of Sussex, Brighton, United Kingdom

${ }^{151}$ School of Physics, University of Sydney, Sydney, Australia

${ }^{152}$ Institute of Physics, Academia Sinica, Taipei, Taiwan

${ }^{153}$ Department of Physics, Technion: Israel Institute of Technology, Haifa, Israel

${ }^{154}$ Raymond and Beverly Sackler School of Physics and Astronomy, Tel Aviv University, Tel Aviv, Israel

${ }^{155}$ Department of Physics, Aristotle University of Thessaloniki, Thessaloniki, Greece

${ }^{156}$ International Center for Elementary Particle Physics and Department of Physics, The University of Tokyo, Tokyo, Japan

${ }^{157}$ Graduate School of Science and Technology, Tokyo Metropolitan University, Tokyo, Japan

${ }^{158}$ Department of Physics, Tokyo Institute of Technology, Tokyo, Japan

${ }^{159}$ Department of Physics, University of Toronto, Toronto, Ontario, Canada

${ }^{160 \mathrm{a}}$ TRIUMF, Vancouver, British Columbia, Canada

${ }^{160 \mathrm{~b}}$ Department of Physics and Astronomy, York University, Toronto, Ontario, Canada

${ }^{161}$ Faculty of Pure and Applied Sciences, University of Tsukuba, Tsukuba, Japan

${ }^{162}$ Department of Physics and Astronomy, Tufts University, Medford, Massachusetts, USA

${ }^{163}$ Centro de Investigaciones, Universidad Antonio Narino, Bogota, Colombia

${ }^{164}$ Department of Physics and Astronomy, University of California Irvine, Irvine, California, USA

${ }^{165 a}$ INFN Gruppo Collegato di Udine, Trieste, Italy

${ }^{165 \mathrm{~b}}$ ICTP, Trieste, Italy

${ }^{165 \mathrm{c}}$ Dipartimento di Chimica, Fisica e Ambiente, Università di Udine, Udine, Italy

${ }^{166}$ Department of Physics, University of Illinois, Urbana, Illinois, USA

${ }^{167}$ Department of Physics and Astronomy, University of Uppsala, Uppsala, Sweden

${ }^{168}$ Instituto de Física Corpuscular (IFIC) and Departamento de Física Atómica, Molecular y Nuclear and Departamento de Ingeniería Electrónica and Instituto de Microelectrónica de Barcelona (IMB-CNM), University of Valencia and CSIC, Valencia, Spain

${ }^{169}$ Department of Physics, University of British Columbia, Vancouver, British Columbia, Canada

${ }^{170}$ Department of Physics and Astronomy, University of Victoria, Victoria, British Columbia, Canada

${ }^{171}$ Department of Physics, University of Warwick, Coventry, United Kingdom

${ }^{172}$ Waseda University, Tokyo, Japan

${ }^{173}$ Department of Particle Physics, The Weizmann Institute of Science, Rehovot, Israel 
${ }^{174}$ Department of Physics, University of Wisconsin, Madison, Wisconsin, USA

${ }^{175}$ Fakultät für Physik und Astronomie, Julius-Maximilians-Universität, Würzburg, Germany

${ }^{176}$ Fachbereich C Physik, Bergische Universität Wuppertal, Wuppertal, Germany

${ }^{177}$ Department of Physics, Yale University, New Haven, Connecticut, USA

${ }^{178}$ Yerevan Physics Institute, Yerevan, Armenia

${ }^{179}$ Centre de Calcul de l'Institut National de Physique Nucléaire et de Physique des Particules (IN2P3), Villeurbanne, France

${ }^{\mathrm{a}}$ Deceased.

${ }^{\mathrm{b}}$ Also at Department of Physics, King's College London, London, United Kingdom.

${ }^{\mathrm{c}}$ Also at Laboratorio de Instrumentacao e Fisica Experimental de Particulas-LIP, Lisboa, Portugal.

${ }^{\mathrm{d} A l s o}$ at Institute of Physics, Azerbaijan Academy of Sciences, Baku, Azerbaijan.

${ }^{\mathrm{e}}$ Also at Faculdade de Ciencias and CFNUL, Universidade de Lisboa, Lisboa, Portugal.

${ }^{\mathrm{f}}$ Also at Particle Physics Department, Rutherford Appleton Laboratory, Didcot, United Kingdom.

${ }^{\mathrm{g}}$ Also at TRIUMF, Vancouver, British Columbia, Canada.

${ }^{\mathrm{h}}$ Also at Department of Physics, California State University, Fresno, CA, USA.

${ }^{i}$ Also at Novosibirsk State University, Novosibirsk, Russia.

${ }^{\mathrm{j}}$ Also at Department of Physics, University of Coimbra, Coimbra, Portugal.

${ }^{k}$ Also at Università di Napoli Parthenope, Napoli, Italy.

${ }^{1}$ Also at Institute of Particle Physics (IPP), Canada.

${ }^{\mathrm{m}}$ Also at Department of Physics, Middle East Technical University, Ankara, Turkey.

${ }^{\mathrm{n}}$ Also at Louisiana Tech University, Ruston, LA, USA.

${ }^{\circ}$ Also at Dep Fisica and CEFITEC of Faculdade de Ciencias e Tecnologia, Universidade Nova de Lisboa, Caparica, Portugal.

${ }^{\mathrm{p}}$ Also at CPPM, Aix-Marseille Université and CNRS/IN2P3, Marseille, France.

${ }^{\mathrm{q}}$ Also at Department of Physics and Astronomy, Michigan State University, East Lansing, MI, USA.

${ }^{\mathrm{r}}$ Also at Department of Financial and Management Engineering, University of the Aegean, Chios, Greece.

${ }^{\mathrm{s}}$ Also at Institucio Catalana de Recerca i Estudis Avancats, ICREA, Barcelona, Spain.

${ }^{t}$ Also at Department of Physics, University of Cape Town, Cape Town, South Africa.

${ }^{\mathrm{u}}$ Also at CERN, Geneva, Switzerland.

${ }^{\mathrm{v}}$ Also at Institut für Experimentalphysik, Universität Hamburg, Hamburg, Germany.

${ }^{w}$ Also at Manhattan College, New York, NY, USA.

${ }^{\mathrm{x}}$ Also at Institute of Physics, Academia Sinica, Taipei, Taiwan.

${ }^{\mathrm{y}}$ Also at School of Physics and Engineering, Sun Yat-sen University, Guanzhou, China.

${ }^{\mathrm{z}}$ Also at Academia Sinica Grid Computing, Institute of Physics, Academia Sinica, Taipei, Taiwan.

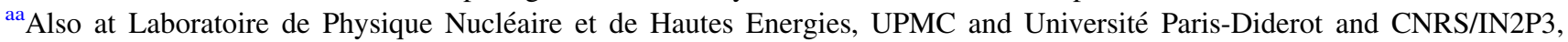
Paris, France.

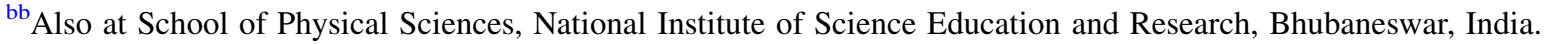

${ }^{\mathrm{cc}}$ Also at Dipartimento di Fisica, Università La Sapienza, Roma, Italy.

dd Also at DSM/IRFU (Institut de Recherches sur les Lois Fondamentales de l'Univers), CEA Saclay (Commissariat à l'Energie Atomique et aux Energies Alternatives), Gif-sur-Yvette, France.

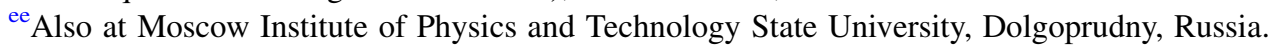

${ }^{\mathrm{ff}}$ Also at Section de Physique, Université de Genève, Geneva, Switzerland.

${ }^{\mathrm{gg}}$ Also at Departamento de Fisica, Universidade de Minho, Braga, Portugal.

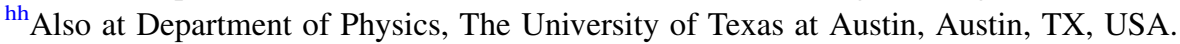

${ }^{i i}$ Also at Institute for Particle and Nuclear Physics, Wigner Research Centre for Physics, Budapest, Hungary.

${ }^{\mathrm{jj}}$ Also at DESY, Hamburg and Zeuthen, Germany.

${ }^{\mathrm{kk}}$ Also at International School for Advanced Studies (SISSA), Trieste, Italy.

${ }^{11}$ Also at Department of Physics and Astronomy, University of South Carolina, Columbia, SC, USA.

${ }^{\mathrm{mm}}$ Also at Faculty of Physics, M.V. Lomonosov Moscow State University, Moscow, Russia.

${ }^{\mathrm{nn}}$ Also at Nevis Laboratory, Columbia University, Irvington, NY, USA.

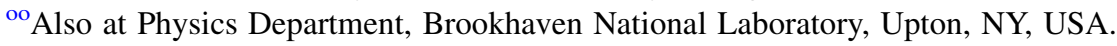

${ }^{\mathrm{pp}}$ Also at Department of Physics, Oxford University, Oxford, United Kingdom.

${ }^{\mathrm{qq}}$ Also at Department of Physics, The University of Michigan, Ann Arbor, MI, USA.

${ }^{r r}$ Also at Discipline of Physics, University of KwaZulu-Natal, Durban, South Africa. 